Inheritance of microspore embryogenic potential and direct embryo to plant conversion in the oilseed rape $\mathrm{DH}$ population DH4079 x Express 617

Ariana Istar Valdés Velázquez

Göttingen, 2016 


\title{
Inheritance of microspore embryogenic potential and direct embryo to plant conversion in the oilseed rape DH population DH4079 x Express 617
}

\author{
Dissertation \\ to obtain the Ph. D. degree \\ in the International Ph. D. Program for Agricultural Sciences in Goettingen (IPAG) \\ at the Faculty of Agricultural Sciences, \\ Georg-August-University Göttingen, Germany
}

presented by

Ariana Istar Valdés Velázquez

born in Mexico City, Mexico

Göttingen, November 2016 
1. Name of supervisor: Dr. Christian Möllers

2. Name of co-supervisor: Prof. Dr. Petr Karlovsky

Date of dissertation: $17^{\text {th }}$ November 2016 


\section{Acknowledgments}

Prima facea, I would like to express my most sincerely gratitude to my supervisor, Dr. Christian Möllers, whose teaching, kindness, support and affection have guided the development of this research. Thank you Christian for being an example of continuous work and generosity. Also, I would like to thank Prof. Dr. Heiko Becker, for his constant support to improve my performance and for giving me the opportunity to join the Plant Breeding Division. I would also like to express my gratitude to Prof. Dr. Wolfgang Link, Dr. Wolfgang Ecke and Dr. Antje Schierholt, for their support and opportune suggestions for the development of my studies. I would like to express my gratitude to Prof. Dr. Petr Karlovsky for kindly consenting to be part of the examination committee.

I show my thankfulness to Uwe Ammermann, Carmen Mensch and Rosemarie Clemens for their technical assistance. I also appreciate the enormous help of Ershad Tavakol, for his kind assistance, and of Dr. Mohamed Iqbal for his supportive suggestions and comments. I am very grateful to Frau Sabine Hippe for her countless help and support.

I would like to express my most sincere gratitude and recognition to Dr. Pilar Soengas, Misión Biológica de Galicia, Spain, for her teaching, tremendous support and appropriate suggestions during the development of this research.

The financial support from the Deutsche Forschungsgemeinschaft (DFG) is extremely appreciated. Special recognition is given to CONACYT and DAAD, who have financed my studies in Germany.

My deepest thanks go to Rosemarie Clemens, who has been my teacher, beloved friend and support in every step along my way. Rosi, thank you for all your fondness, which is entirely reciprocal. This work is also yours. We are a great team!

I would like to sincerely thank my colleagues and mates for making the institute a pleasant place: Katharina Ziese-Kubon, Anja Herdlitschke, Gregor Welna, Julia Rudloff, Sasanti Widiarsih, Eva Heinrich, Nora Aqtbouz, Lisa Brünjes, Winda Puspitasari and 
Rahul Raj. My biggest thanks to Daniel Siebrecht, Edy Suprianto, Jan-Christoph Richter, Mathias Starke, Michael Ruland, Sebastian Miersch and Siyang Liu for their support and friendship, especially for each shared laugh along the way. Christopher Hoppe, special thanks to you for our morning talks, laughter, support, joint air and space! My deepest thanks to you Haiko Brandes, for every minute and joy in the journey; without you this work would not have been possible. My sincere gratitude goes to my lovely and amazing soul mates Luisa Pfalsdorf, Mareile Stever, Lishia Teh, Nina Behnke and Rosa Agneta for walking hand in hand together!

Likewise, I would like to thank my greatly beloved companions of life Betzy, Ingrid, Dalia, Blanca and Adrian, whom I deeply love and have been for many years supporting me unconditionally. I express my great gratitude to my second family: Lupita, Valeria, Denisse, Mary, Nic, Aura, Ramon, Tania y Miguel and my nephews that opened their hearts to make me feel at home. Thanks to Anita, Gaby, Rosalia and Verna for all the beautiful moments together; I definitely couldn't have done it without my Mexicans. Especially, I express my deep gratitude and affection to my two marvelous friends, Sandra and Jhenny that turned my life in a wave of irrepressible laughter!

Finally and certainly significant, I would like to acknowledge and dedicate this work to my beloved family, my parents, Cesar and Hortensia, for their unconditional love and support, for their extraordinary example of perseverance and dedication; my brothers, Ernesto and Frida, to grandma Catalina, Marena and Angeles, Argelia and Luna, Miris Yaros and Tana, Paty y Ernesto for being my daily motivation; to the Vergara-García; to Ephedra.

Dedicated to Lily and Eri. 


\section{Table of Contents}

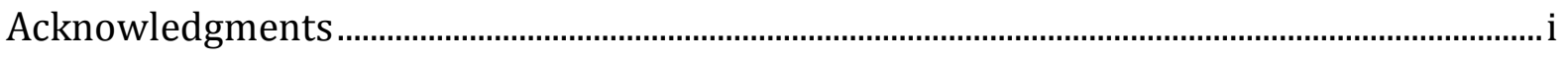

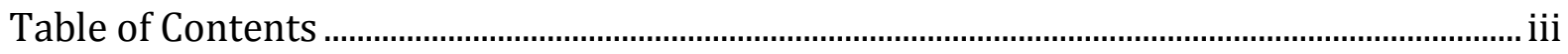

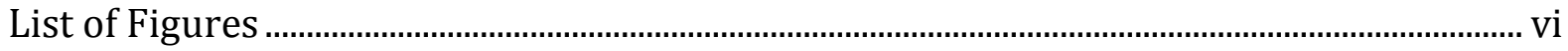

List of Tables

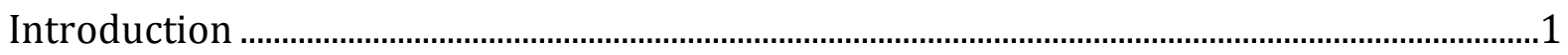

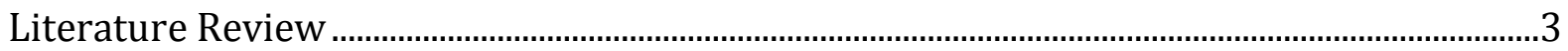

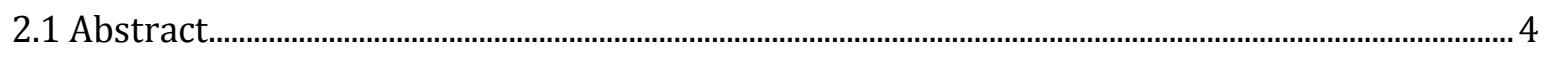

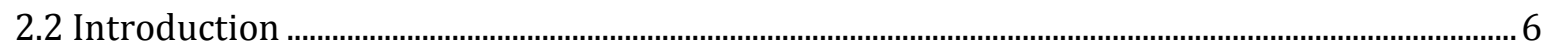

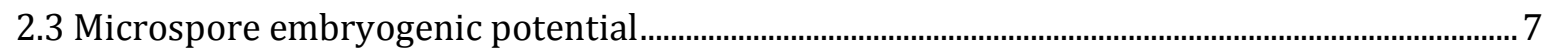

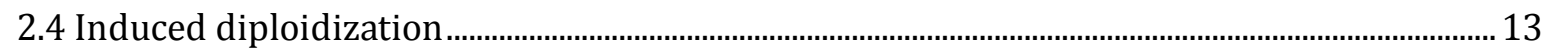

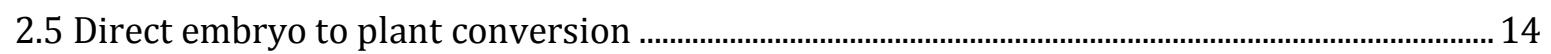

2.6 Inheritance of microspore embryogenic potential, diploidization and direct embryo to

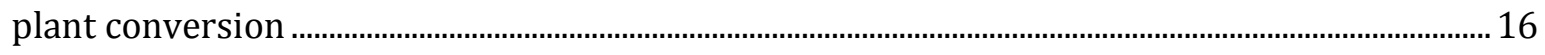

2.7 Identification of candidate genes for microspore culture response ............................................. 19

Phenotypic variation and QTL mapping of microspore embryogenic potential and direct embryo to plant conversion in the DH population DH4079 x Express (Brassica napus L.)

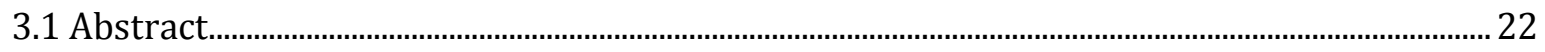

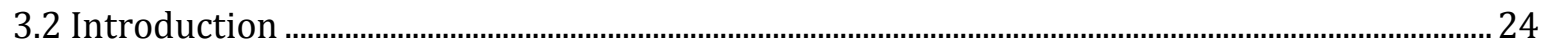

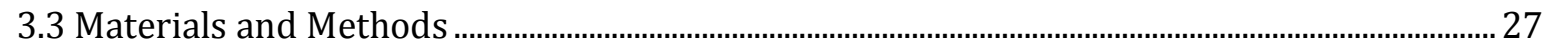

3.3.1 Development of the DH Population ................................................................................................. 27

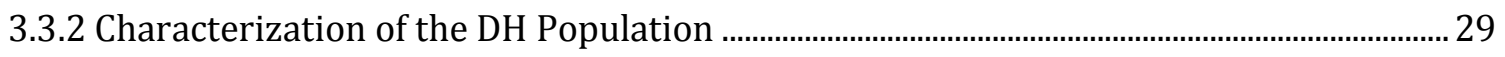

3.3.3 Experimental design and Statistical Analysis ....................................................................... 30 
3.3.4 SNP Marker Analysis and Linkage Map Development

3.3.5 QTL Analysis and Study of Distorted Marker Segregation......................................................... 32

3.3.6 Synteny Analysis for Candidate Gene Localization ...................................................................... 32

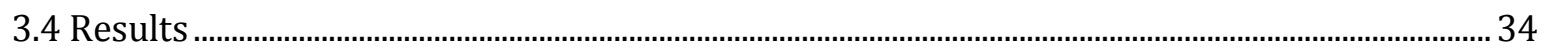

3.4.1 Phenotypic Analysis and Heritability .......................................................................................... 34

3.4.2 Correlation among Traits ….................................................................................................. 41

3.4.3 Genetic Map and Marker Segregation........................................................................................... 42

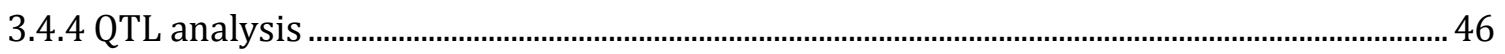

3.4.5 Identification of candidate genes within QTL regions ......................................................... 51

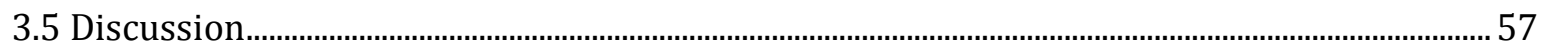

3.5.1 Phenotypic variation in the DH4079 x Express 617 population ............................................ 57

3.5.2 Distorted segregation in the DH4079 x Express 617 DH population ................................... 60

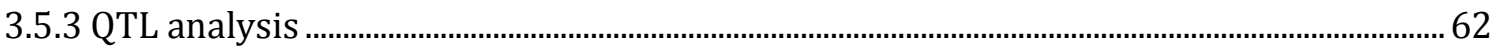

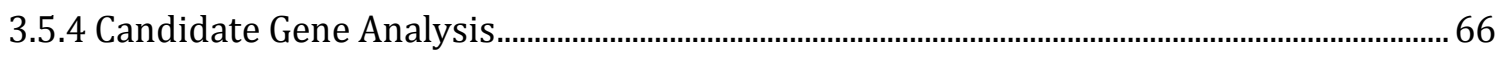

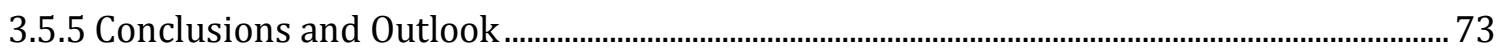

Effect of a low temperature treatment on gene expression in microspore derived

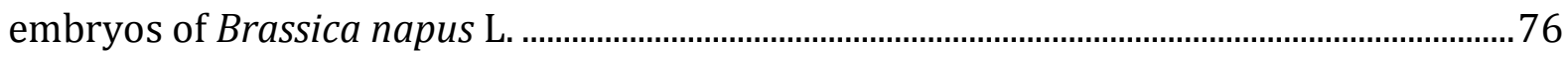

4.1 Abstract

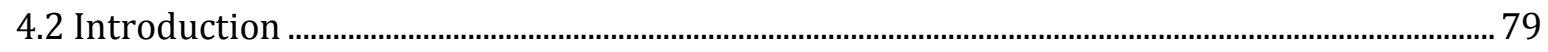

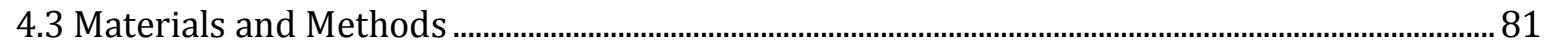

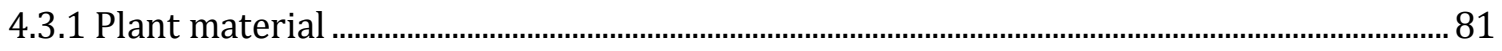

4.3.2 MACE

4.3.3 Gene annotation and gene expression profiling................................................................... 82

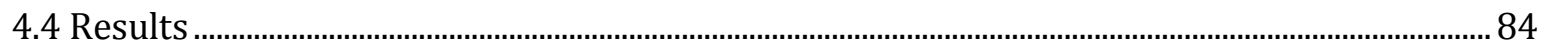

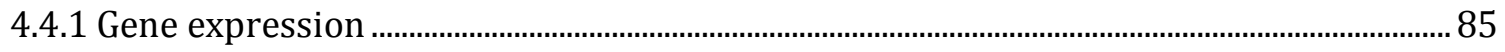

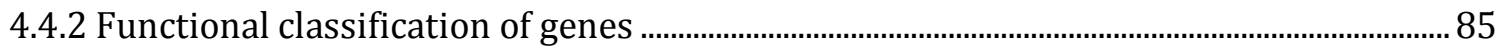


4.5 Discussion. 94

4.5.1 Gene Expression and Functional Classification. 94

4.5.2 Differential genes expression profiling. 94

4.5.3 Conclusions and Outlook 98

General Discussion 99

5.1 Genetic Variation in microspore culture response of the DH4079 x Express Population... 99

5.2 Distorted segregation and identification of QTL for microspore culture response............. 100

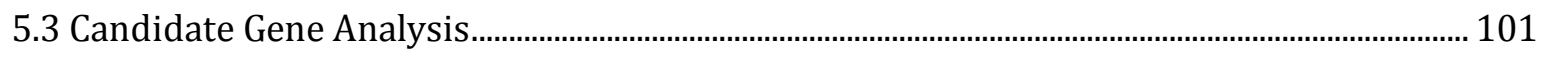

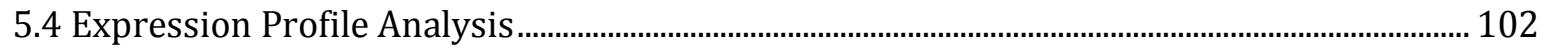

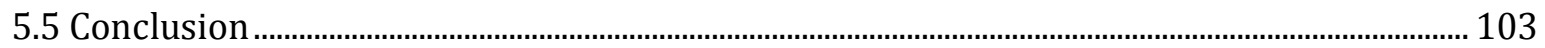

Summary

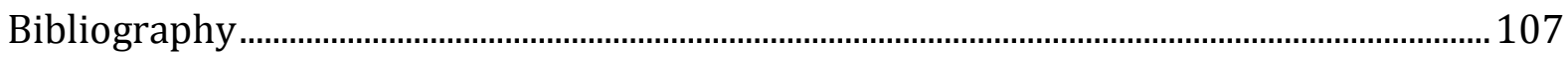

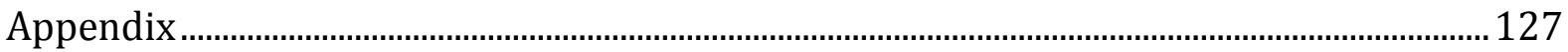

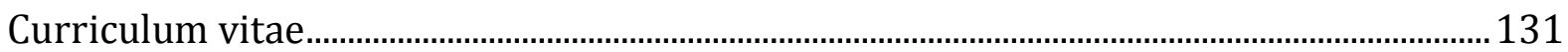




\section{List of Figures}

Figure 3.1 Frequency distribution of the direct embryo to plant conversion of the segregating microspore derived embryo population derived from the F1 DH4079 x Express 617

Figure 3.2 Highly different responses to microspore culture: a) morphological variation among genotypes of the DH population, b) quantity of microspore derived embryos produced by parental lines Express 617 (above) and DH4079 (below), and c) secondary embryogenesis response (above) and direct embryo to plant conversion (below).

Figure 3.3 Frequency distribution of the residuals (left) and the traits (right) microspore density, embryogenic potential and survival of embryos of the DH population. Residual values are normally distributed. Frequency distribution of residuals of embryogenic potential was generated based on square root transformed data. Mean values of the DH population (vertical dashed line), of the cross (F1) and parental genotypes Express 617 (Ex617) and DH4079 (DH4079) are also presented.

Figure 3.4 Frequency distribution of the residuals (left) and of the traits (right) direct embryo to plant conversion, root regeneration, and secondary embryogenesis of the DH population. Residual values are normally distributed. Mean values of the DH population (vertical dashed line), of the cross (F1) and parental genotypes Express 617 (Ex617) and DH4079 (DH4079) are also presented.

Figure $\quad 3.5$ Distribution of distorted marker segregation along the 19 linkage groups in the DH population DH4079 x Express 617 (black line). Distorted marker segregation of the 4 subpopulations is defined by colored lines. Significant skewed marker segregation $(\mathrm{P}<0.05)$ above the absolute Chi-squared value $\left(x^{2}=3.84\right)$ is represented by a dashed red line. Alleles segregating in favor of parental genotype DH4079 have positive values, alleles segregating in favor of parental genotype Express 617 have negative values.

Figure 3.5 (Continue from previous page) Distribution of distorted marker segregation areas along the 19 linkage groups in the DH population DH4079 x Express 617 (black line). Distorted marker segregation of the 4 subpopulations is 
defined by colored lines. Significant skewed marker segregation $(\mathrm{P}<0.05)$ above the absolute Chi-squared value $\left(\mathrm{x}^{2}=3.84\right)$ is represented by a dashed red line. Alleles segregating in favor of parental genotype DH4079 have positive values, alleles segregating in favor of parental genotype Express 617 have negative values. 45

Figure 3.6 Positions of QTL for the traits microspore embryogenic potential (POT), survival (SURV), direct embryo to plant conversion (DEPC) and root regeneration (ROOT). QTL for the traits survival, direct embryo to plant conversion and root regeneration showed overlapping confidence intervals in linkage group A05. Genetic positions of markers flanking genomic regions related to the QTL are shown (bold).

Figure $\quad 3.7$ Molecular linkage map of the DH population DH4079 x Express 617. Marker segregation, mapped QTL and their most likely positions are shown 50

Figure $\quad 3.8$ QTL positioned in linkage groups A01 and C06 associated with the trait microspore embryogenic potential (POT.A01.67 and POT.C06.7134) in the genetic map of B. napus. Physical positions of possible candidate genes within the SNP markers (bold) delimiting the $95 \%$ confidence interval of the QTL for microspore embryogenic potential in the map of B. rapa and B. oleracea are shown.

Figure 3.9 QTL positioned in linkage groups A05 and A10 associated with the traits survival (SURV.A05.43), direct embryo to plant conversion (DEPC.A05.41), root (RO0T.A05.41) regeneration and secondary embryogenesis (SEEM.A05.21, SEEM.A10.20) in the genetic map of B. napus. Physical positions of possible candidate genes within the SNP markers (bold) delimiting the $95 \%$ confidence interval of the QTL for direct embryo to plant conversion and secondary embryogenesis in the map of $B$. rapa are shown

Figure 3.10 QTL positioned in linkage group A02 associated with the traits, microspore embryogenic potential (POT.A02.73) and direct embryo to plant conversion (DEPC.A02.49) in the genetic map of B. napus. Physical positions of possible candidate genes within the SNP markers (bold) delimiting the $95 \%$ confidence interval of the QTL for direct embryo to plant conversion in the map of B. rapa are shown. .56

Figure $\quad 3.11$ QTL positions in linkage group A05 associated with the traits survival (SURV), direct embryo to plant conversion (DEPC) and root (ROOT) regeneration showed overlapping confidence intervals in the genetic map 
of B. napus. Physical positions in the B. rapa map within the $95 \%$ confidence interval of the QTL accounting for DEPC and SECEMB are shown (red), as well as physical positions of markers flanking putative genomic regions related to microspore embryogenic potential (black) and DEPC (grey) according to Ecke et al. (2015) and Kampouridis et al. (2016)..........64

Figure 4.1 Genes expressed in control and cold treated embryos in Express 617 (E) and DH4079 (T) genotypes of B. napus. 50,630 genes were simultaneously detected in each of the four libraries and 1,970 genes were specifically expressed in cold treated embryos of both genotypes.

Figure 4.2 Gene ontology (GO) classification of annotated genes in two main GO categories: molecular function and biological process. The number of genes ( $\mathrm{x}$-axis) belonging to the most representative 21 sub-categories (y-axis) are shown 86

Figure 4.3 Hierarchical cluster analysis of the 2,606 differentially expressed genes in cold treated embryos compared to control embryos from two different rapeseed genotypes, Express 617 and DH4079 (columns). Similar expression patterns in both genotypes are shown (arrows). Hierarchical cluster analysis was based on log2FoldChange values, displaying expression patterns of genes (rows). The expression of a gene was significantly different over a threshold of 2.3 log2FoldChange absolute value. Green palette represents up-regulated expression and red palette represents down-regulated expression .88

Figure 4.4 Differentially expressed genes (>log2FoldChange 2.3) of cold treated embryos $(p<0.05, \mathrm{FDR}<0.05)$ in comparison to control embryos: 539 genes were exclusively differentially regulated (up- or down- regulated) in Express 617 (E-Treated) and 1,547 in DH4079 (T-Treated), whereas 187 genes were differentially expressed in both genotypes.

Figure 4.5 Direction of gene regulation in differentially expressed transcripts identified in cold treated embryos in comparison to control embryos in genotypes Express 617 and DH4079. a) A total of 726 genes were differentially expressed in Express 617 and 1,734 in DH4079. b) 187 genes were simultaneously modulated in both genotypes, even though the direction of the gene regulation was not identical in Express 617 and DH4079 for all genes, as revealed by the different percentages shown for the up-and down-regulated expression. 


\section{List of Tables}

Table 3.1 Variance components and heritabilities of the DH population

Table 3.2 Descriptive statistics of the studied variables in the DH population, parental lines and progeny.

Table 3.3 Spearman's rank correlations $\left(\mathrm{r}_{\mathrm{S}}\right)$ between microspore culture traits 41

Table 3.4 Marker distribution, size and mean distances between markers of each linkage group of the $\mathrm{A}$ and $\mathrm{C}$ genomes in the linkage map of the $\mathrm{DH}$ population DH4079 x Express 617. 43

Table 3.5 Mapped QTL and their most likely positions for the studied traits in the DH population DH4079 x Express 617 48

Table 3.6 Candidate genes of $B$. rapa and B. oleracea located within the $95 \%$ confidence interval of the QTL accounted for microspore embryogenic potential. 51

Table 3.7 Candidate genes of B. rapa and located within the $95 \%$ confidence interval of the QTL accounted for secondary embryogenesis.

Table 3.8 Candidate genes of B. rapa and located within the $95 \%$ confidence interval of the QTL accounted for direct embryo to plant conversion .55

Table 4.1 Summary of statistics of mRNA reads in the four libraries of B. napus microspore derived embryos.

Table 4.2 Top ten up-regulated transcripts in B. napus cold treated microspore derived embryos of Express 617 and DH4079 genotypes.

Table 4.3 Top ten down-regulated transcripts in B. napus cold treated microspore derived embryos of Express 617 and DH4079 genotypes. 93 


\section{Chapter 1}

\section{Introduction}

Oilseed rape (Brassica napus L., AACC, $2 \mathrm{n}=38$ ) is an amphidiploid species belonging to the family Cruciferae (Brassicaceae). It is one of the most economically important and exhaustively studied oil and protein crops worldwide. The geographical origin of rapeseed is assumed to be in the European-Mediterranean region through the natural interspecific hybridization of the diploid species $B$. rapa (AA genome, 2n $=20$ ) and $B$. oleracea (CC genome, 2n = 18) (Downey and Rimmer 1993).

Among dicotyledonous species, oilseed rape (B. napus L.) and tobacco are model plants for microspore embryogenesis, i.e. the in vitro regeneration of plantlets from immature pollen grains. This applies all the more for oilseed rape because this species is closely related to the model plant Arabidopsis thaliana, which up to date belongs to the nonresponsive species with respect to microspore embryogenesis (Hosp et al. 2007). Among the Brassica species B. napus is considered to be the most embryogenic one (Ferrie 2003).

During the past 20 years, microspore culture in oil seed rape has become increasingly important in commercial breeding programs for the fast development of completely homozygous doubled haploid lines. Nowadays, doubled haploid technology is considered as one of the most important techniques to achieve success in plant breeding programs since recessiveness recovery is accomplished, traits are rapidly fixed and selection efficiency is increased (Steward 2008; Ali et al. 2009). Besides that, double haploid populations are employed for the construction of linkage maps and QTL analysis (Yang et al. 2014). 
Microspore culture efficiency depends on important factors, such as donor plant developmental stage and growth conditions, microspore developmental stage and pretreatment, microspore plating density, nutrient content of culture medium and culture conditions (Möllers and Iqbal 2009). Despite the progress achieved in improving the androgenesis lab protocols, the outcome of microspore culture has been proven to be still very much genotype-dependent (Ferrie and Möllers 2011). Although a number of studies aimed at identifying the genetic causes of the enormous differences in the microspore embryogenic potential and the capacity of the embryos to directly convert into plantlets of different oilseed rape genotypes, the genetic causes remain a mystery. A continuous improvement in the microspore culture technology is vital for a competitive generation of doubled haploid populations in commercial breeding programs. As pointed out earlier (Möllers and Iqbal 2009), there are still some shortages at three major steps of the whole process: (1) microspore embryogenic potential, (2) induced diploidization and (3) direct embryo to plant conversion. Identifying the genetic causes for mentioned limitations in microspore embryogenic potential and direct embryo to plant conversion could help to improve the system through marker assisted selection of genotypes with improved androgenic potential. It may also contribute by suggesting which medium components should be modified to achieve a better response.

The objectives of the present work were

(1) to develop a doubled haploid population from the cross of the highly embryogenic line DH4079 and the low embryogenic inbred line Express 617

(2) to characterize the generated doubled haploid population for its microspore embryogenic potential, its direct embryo to plant conversion as well as for other relevant traits

(3) to develop an Illumina SNP-chip based molecular marker map, to identify QTL for those traits and to localize candidate genes within QTL confidence intervals for the traits of interest, and

(4) to study the gene expression pattern in low-temperature treated microspore derived embryos with the aim to identify differentially expressed genes which may be involved in the enhanced direct embryo to plant conversion after this treatment 
Chapter 2

Literature Review 


\subsection{Abstract}

Brassica napus L. has become a model plant for studying microspore embryogenesis. Nevertheless, the genetic causes of the enormous differences in the microspore embryogenic potential of oilseed rape genotypes remains unknown and a continuous enhancement in the microspore culture success is of great importance for the efficient generation of doubled haploid populations in commercial breeding programs. The present chapter summarizes the considerable research in microspore culture that has been accomplished in recent years and points at open questions that may be addressed in in upcoming studies. An enhancement for embryo yield has been achieved by the addition of substances with an effect on key processes during the embryo development, such as ethylene biosynthesis inhibitors (antioxidant activity), brassinosteroids (establishment and maintenance of the apical meristem) and bleomycin (cytoskeletal reorganization). Supplementary stressing factors (temperature, $\mathrm{pH}$ ) in further research would enable the application of new efficient methods in oilseed rape microspore culture. A low frequency of spontaneous diploidization has been reported in microspore cultures of oilseed rape. The positive effect of colchicine as a mitotic inhibitor is widely recognized besides its enhancing effect on embryo yield. Nevertheless, its toxicity has led to the evaluation of possible synergistic effects of a combination of less harmful substances, for instance APM, pronamide, oryzalin and trifluralin. An efficient conversion of microspore derived embryos to plants is required for a successful generation of double haploid populations. A main factor involved in direct embryo to plant conversion is the proper development and maintenance of the apical meristem. The addition of brassinolide regulates such process and has been proven to be successful as well as the application of a cold treatment, which significantly increased direct embryo to plant conversion. The inheritance of microspore embryogenic potential and direct embryo to plant conversion is poorly understood in spite of the great effort conducted to identify genes involved in those traits. Several studies assessed the distorted marker segregation pattern in oilseed rape, concluding that a deviation in the expected Mendelian segregation ratio is not randomly distributed along the genome. Skewed marker segregation depends on the studied population and different genomic regions may cause distorted segregation, supporting the assumption 
that such traits are polygenic. The study of gene expression profiles allowed the characterization of a large number of genes which showed an up- or down- regulation during the embryogenic process. Among those genes FUSCA3, LEC1, LEC2, BBM, WOX2 and WOX9, ABSCISIC ACID INSENSITIVE3, CLE, MEE67, HSP70B exhibit a molecular activity strongly related to the ability of microspores to develop into embryos. The genetic basis of the direct embryo to plant conversion is still poorly understood but genes involved in the establishment of cell fate could influence plant development in vitro, for instance, ZLL, AG01, CLV, WUS, WOX, CUC and SCL genes. The significant progress achieved by sequencing the oilseed rape genome and the availability of a large number of informative SNP-markers facilitates the future characterization of genes involved in microspore embryogenesis and direct embryo to plant conversion. 


\subsection{Introduction}

Among dicotyledonous species, oilseed rape (Brassica napus L.) and tobacco are model plants for microspore embryogenesis. This applies all the more for oilseed rape because this species is closely related to Arabidopsis thaliana, which up to date belongs to the non-responsive species with respect to microspore embryogenesis. More than five years have elapsed since the last review about microspore embryogenesis in oilseed rape has been published (Ferrie and Möllers 2011). During this period, important progress has been achieved by sequencing the genome of Brassica napus (Chalhoub et al. 2014) as well as of its direct diploid ancestor species Brassica rapa L. (Wang et al. 2011) and Brassica oleracea (Liu et al. 2014). An Illumina SNP-chip with more than 50.000 SNP-markers has been developed (Clarke et al. 2016) which for the first time allows the identification of physical marker positions in the sequenced genomes and thereby allows the direct comparison of linkage maps derived from crosses involving different genotypes. On the other hand, increasing performance in RNA sequencing technologies (Wang et al. 2009; Bojahr et al. 2016) enabled the analysis of differences in gene expression, which helped to identify candidate genes involved in microspore embryogenesis and direct embryo to plant conversion. Recently developed CRISPR/Cas9 technology (Barrangou and Doudna 2016) promises to provide a tool to specifically inactivate candidate genes and hence may help in proving their functions.

The genetic cause for the enormous differences in the microspore embryogenic potential of different oilseed rape genotypes remains a mystery. The microspore embryogenic potential is defined as the number of microspore derived embryos produced from a certain number of microspores. A continuous improvement in the microspore culture technology is vital for a competitive generation of doubled haploid populations in commercial breeding programs. As pointed out earlier (Möllers and Iqbal 2009), there are shortages at three major steps of the process: (1) microspore embryogenic potential, (2) induced diploidization and (3) direct embryo to plant conversion. Since the pioneering work of Lichter (1982; Lichter 1985; Gland et al. 1988) continuous improvements in different steps of the protocol has been achieved. This manuscript summarizes the progress accomplished with a focus on the past six years and intends to point at open questions that may be approached in future research. It 
also complements recent reviews of Islam and Tuteja (2012) and Soriano et al. (2013) which are not specific for Brassica napus.

\subsection{Microspore embryogenic potential}

The NLN-medium Robert Lichter used in his microspore experiments is the basal medium of Nitsch and Nitsch (1967) which was developed for in vitro cultures of Plumbago indica. Lichter (1985) supplemented this basal medium with L-serine (100 $\mathrm{mg} / \mathrm{l})$ and L-glutamine (800 $\mathrm{mg} / \mathrm{L}$ ) and some phytohormones. The original medium used by Lichter (1985) contains $125 \mathrm{mg} / \mathrm{L}$ magnesium sulphate, even though the frequently used NLN-medium of Duchefa (N0252; https://www.duchefabiochemie.com/product/details/number/ N0252) contains only $61 \mathrm{mg} / \mathrm{L}$. From the published literature it is not clear, how much effort Lichter (1985) made to optimize the composition of the NLN-medium. The results of Gland et al. (1988) do not allow to conclude which of the nine different tested culture medium variants are more suitable for regeneration of a maximum number of embryos. Surprisingly, few studies were made to optimize the culture media composition for Brassica species. The fact that embryo yield was enhanced if the culture medium was replaced by fresh medium after a culture period of three days indicates that at this time the concentration of some of the minor medium constituents is limiting embryo regeneration (Hansen and Svinnset 1993). However, the positive effect of replacing the culture medium after 24-48 h has also been explained by auto-toxic effects of freshly isolated microspores (Kott et al. 1988b; Gu et al. 2003). In order to induce a sporophytic development of microspores, different stresses have been applied, which increased reactive oxygen species (ROS) production (Hoseini et al. 2014), an undesirable effect that could be overcome by the replacement of culture medium.

Leroux et al. (2009) reported that inhibition of ethylene biosynthesis in B. napus by increasing concentrations of cobalt chloride and aminoethoxyvinylglycine (AVG) led to the enhancement in embryo yield of $75 \%$ and $50 \%$ respectively, whereas addition of the ethylene precursor S-adenosyl-methionine or the ethylene-releasing agent ethephon decreased embryo yield. The use of silver nitrate as an ethylene inhibitor resulted in the improvement of anther and microspore responsiveness in $B$. juncea, $B$. 
oleracea and B. rapa (Biddington et al. 1988; Dias and Martins 1999; Malik et al. 2001; Prem et al. 2005; Na et al. 2011; Kabir et al. 2013). Several authors included activated charcoal on Brassicas microspore culture protocols because of its ability to catch toxic compounds as ethylene (e.g. Gland et al. 1988; Mathias 1988; Lichter 1989; Ferrie 2003; Gu et al. 2004; Supena et al. 2006; Jiang et al. 2008; Prem et al. 2008; Takahira et al. 2010). However, its inhibitory activity in microspore embryogenesis has also been observed (Yang et al. 2014). Activated charcoal can irreversibly adsorb a large range of substances, including vitamins, metal ions and plant growth regulators (Thomas 2008), reason why its effects on microspore culture are still unclear.

In barley, increased concentration of the micronutrient copper sulphate in anther culture has improved the frequency of responding anthers and of regenerated green plantlets (Nuutila et al. 2000; Wojnarowiez et al. 2002; Jacquard et al. 2009). The positive effect of copper sulphate may rely on its ability to enhance microspore survival and synchronization of the first embryogenic division (Wojnarowiez et al. 2002), but this effect has not yet been investigated in Brassica microspore culture.

Hormones are involved in every process of plant development. Nevertheless, their addition is omitted nowadays from current microspore culture protocols, reinforcing the commonly accepted knowledge that microspore embryogenesis in Brassica napus works as efficient without the addition of any phytohormone. However, a proper balance among growth regulators is required to trigger microspore embryogenesis (Zur et al. 2014). The use of brassinosteroids, important steroidal growth regulators, to enhance rapeseed microspore embryogenic potential has been studied by Ferrie et al. (2005). Two brassinosteroids, 24-epibrassinolide and brassinolide, were proved to increase up to twelve-fold and ten-fold embryogenesis in B. napus and B. juncea respectively, including recalcitrant oilseed rape cultivars. Such results were confirmed by Belmonte et al. (2010), who identified a two- to five-fold enhancement on microspore derived embryo production and better embryo quality, characterized by the development of zygotic-like shoot apical meristems, when cultures were treated with brassinolide, while the addition of the brassinolide biosynthesis inhibitor brassinazole substantially decreased embryo yield and promoted the formation of aberrant apical meristems. Ferrie et al. (2005) postulated the brassinolide protective effect on microspores to the initial high-temperature treatment since its activity against biotic 
and abiotic stresses has already been suggested (Krishna 2003; Divi and Krishna 2009). Otherwise, brassinolide could have a positive influence on microspore culture due to its ability to modulate glutathione and ascorbate metabolism, shifting redox homeostasis from a reduced to an oxidized ratio during microspore culture process (Belmonte et al. 2010) that favors embryo yield and apical meristem development (Belmonte et al. 2006; Stasolla et al. 2008; Stasolla 2010). The enhanced microspore embryogenic potential effect of the exogenous addition of ascorbic acid and glutathione was studied by Hoseini et al. (2014). At a concentration of $10 \mathrm{mg} / \mathrm{l}$ of ascorbic acid, embryo yield in B. napus was almost doubled in contrast to control cultures. Ascorbic acid activity was increased in a combination treatment with $50 \mathrm{mg} / \mathrm{l}$ of $\alpha$-Tocopherol, an organic compound from the Vitamin E family, or in conjunction with $10 \mathrm{mg} / \mathrm{l}$ of reduced glutathione. In B. campestris the induction of microspores in glutathione absence decreased embryo yield (Yang et al. 2014), suggesting a potential positive effect of glutathione in microspore embryogenesis.

The effect of exogenous auxins on androgenesis of oilseed rape and other Brassica species has been shown to be detrimental and embryo yield is significantly decreased in cultures treated with 2,4-dichlorophenoxyacetic acid (2,4-D), 1-naphthaleneacetic acid (NAA) and indole-3-acetic acid (IAA) (Ramesar-Fortner and Yeung 2006; Ardebili and Mehran 2011; Yang et al. 2014). Supporting these results, the addition of antiauxins has resulted in improved microspore embryogenesis. In $B$. juncea, microspores treated with $20 \mu \mathrm{M}$ of the antiauxin $p$-chlorophenoxyisobutyric acid (PCIB) showed an up to five-fold improved embryogenesis at a higher induction temperature of $35{ }^{\circ} \mathrm{C}$ in comparison to colchicine treated cultures (Agarwal et al. 2006). Likewise, in B. rapa, $40 \mu \mathrm{M}$ of PCIB increased the embryo yield by three- to six-fold over the controls (Zhang et al. 2011). The effect of PCIB was also studied in B. napus, in which a three- to four-fold higher embryo yield was obtained when microspores were incubated for $20 \mathrm{~min}$ in the presence of 3 and $4 \mathrm{mg} / \mathrm{l}$ of PCIB (Ahmadi et al. 2012).

In contrast to auxins, exogenous cytokinins have shown to enhance androgenesis in Brassica species. Zhang et al. (2012) reported a significant increase of embryo yield in $B$. rapa when microspores of low and high responsive genotypes were cultured in the presence of minimal 6-benzylaminopurine (BA) concentrations (0.05-0.1 mg/l). At higher BA concentrations the embryo yield decreased. Similar observations were found 
by Takahashi et al. (2012). The addition of $0.1 \mathrm{mg} / \mathrm{l}$ and $0.3 \mathrm{mg} / \mathrm{l}$ of BA enhance two- to twelve-fold embryo yield in five B. rapa subspecies. In two cultivars of B. campestris, the addition of BA (0.4 mg/l) and zeatin ( $0.2 \mathrm{mg} / \mathrm{l})$ to microspore culture improved embryo yield(Yang et al. 2014). To some extent, a low auxin/cytokinin ratio seems to be required at the early stages of the androgenic process, but a shift in phytohormone balance during culture could be suitable for the proper development and maturation of embryos as observed in vivo and in vitro since the equilibrium among growth regulators has shown to be a major factor influencing microspore culture success, more than the actual concentration of individual hormones (Hays et al. 2001; Zur et al. 2014; Dubas et al. 2014; Zur et al. 2015). In another study of rapeseed, microspore embryogenic efficiency was improved by exogenous abscisic acid (ABA) at a concentration of 0.5 $\mathrm{mg} / \mathrm{l}$ for $12 \mathrm{~h}$ which enhanced embryo yield three-fold compared to untreated cultures and increased plantlet regeneration by $68 \%$ (Ahmadi et al. 2014). In a recent publication, Dubas et al. (2013) detected an increase in endogenous ABA concentration as a consequence of heat treatment of microspores, underlining the importance of $A B A$ on microspore embryogenesis, an issue that so far is poorly understood.

Ahmadi et al. (2014) studied the effect of jasmonic acid (JA) and salicylic acid (SA) on microspore cultures of B. napus. $1.0 \mathrm{mg} / \mathrm{l}$ of JA and $0.2-0.5 \mathrm{mg} / \mathrm{l}$ of SA positively influenced microspore embryogenesis compared to control, when microspores were treated for $24 \mathrm{~h}$ and $6 \mathrm{~h}$ respectively.

Embryogenesis induction in microspore culture is a stress-dependent process (Touraev et al. 1997) and besides enhancing embryo diploidization, anti-mitotic drugs act as stress factors triggering the sporophytic development of microspores since alteration of cytoskeleton homeostasis is considered of great significance for embryogenic success (Simmonds and Keller 1999). Colchicine is an antimicrotubular substance widely used in microspore culture protocols of Brassica species and is able to induce microspore embryogenesis without heat treatment (Zhao et al. 1996a), and since decades, its ability to enhance embryo yield has been recognized (Iqbal et al. 1994; Chen et al. 1994; Zaki and Dickinson 1995; Zhou et al. 2002a; Zhou et al. 2002b). In a range of 10 to $500 \mathrm{mg} / \mathrm{l}$, colchicine is able to increase microspore embryogenesis when applied for 15 to 72 hours. Colchicine was able to replace the heat shock $\left(32^{\circ} \mathrm{C}\right)$ required for the initiation of embryogenesis in B. napus. Using microspores of B. napus cv. Topas, Zhao et al. (1996) 
induced microspore embryogenesis using the non-inductive temperature of $25{ }^{\circ} \mathrm{C}$ and treating the microspore cultures with $25 \mu \mathrm{M}$ colchicine for $42 \mathrm{~h}$ followed by a dilution to $12.5 \mu \mathrm{M}$ with fresh medium. Klutschewski (2013) studied in detail the influence of colchicine in 17 oilseed rape genotypes, denoting that a treatment of $25 \mu \mathrm{M}$ for $72 \mathrm{~h}$ of colchicine is able to significantly enhance embryogenesis compared to untreated cultures. More prolonged periods of colchicine treatment reduced embryo yields and increased the frequency of tetraploid plants. In the same study, the effect of amiprophos-methyl (APM) and pronamide, mitosis inhibitor substances, was evaluated and resulted in a decreased embryogenic potential in all genotypes compared to colchicine treatments. Reduction of embryo yield by the addition of pronamide and APM may be due their increased affinity to plant microtubules that could maintain microtubule destabilization for a longer period before being degraded. An enhancement of embryo yield related to the antibiotic and antimitotic bleomycin treatment in $B$. napus was reported by Zeng et al. (2010) and Ahmadi et al. (2012). Applying 0.1 to 0.2 $\mu \mathrm{g} / \mathrm{ml}$ bleomycin for 20 to 30 minutes increased embryo production in oilseed rape two- to four-fold (Zeng et al. 2010; Ahmadi et al. 2012). The biochemical effect of bleomycin on oilseed rape microspore culture is still unknown which encourages the application of the antibiotic in further studies. Cytochalasin D is a fungal metabolite, whose addition to induction medium was able to promote microspore embryogenesis in the absence of heat stress by promoting a cytoskeletal reorganization that resulted in symmetric divisions of B. napus microspores (Gervais et al. 2000). Similarly, $n$-Butanol is a compound that induces rearrangements in the microspore cytoskeleton facilitating symmetric divisions that led to embryogenesis (Fábián et al. 2015). The promoting role of $n$-butanol in microspore embryogenesis of maize, barley and wheat has been proved (Soriano et al. 2008; Földesiné Füredi et al. 2011; Földesiné Füredi et al. 2012; Castillo et al. 2014), but it has not yet been assessed in B. napus.

Temperature is considered an important factor controlling the embryogenic development of microspores in B. napus (Custers et al. 1994). An initial $32^{\circ} \mathrm{C}$ heat treatment for 1-2 days followed by an incubation at lower temperature $\left(25-28^{\circ} \mathrm{C}\right)$, has been routinely used as a stress promoter of microspore embryogenesis in Brassica species recalcitrant genotypes (Abraha et al. 2008; Zhang et al. 2012). Besides promoting a cytoskeleton reorganization (Gervais et al. 2000), temperature influences endogenous auxin distribution during microspore embryogenic development in $B$. 
napus and its local accumulation resulting from heat treatment determines embryo polarity and the subsequent axiality of adult plants (Dubas et al. 2014), which is supported by the observation of an enhancement of microspore derived embryos with suspensor-like structures by high-temperature that specifies the apical-basal polarity of embryos (Supena et al. 2008). An initial heat treatment in combination with the addition of antiauxin substances in microspore cultures of B. napus may have a positive effect on embryo yield as has been observed in B. juncea (Agarwal et al. 2006). Prem et al. (2012) reported the successful embryogenesis of oilseed rape microspores at $18^{\circ} \mathrm{C}$ involving the formation of suspensor-like structures, which would be a new system to achieve microspore derived embryo formation in heat sensitive genotypes.

Polyethylene glycol (PEG 4000) has shown to be a successful embryogenic inducer of microspores able to replace sucrose as osmoticum in B. napus cultures (Ilić-Grubor et al. 1998). Ferrie and Keller (2007) reported a significant enhancement of embryogenesis in B. napus and other cruciferous species when microspores were cultivated in a low sucrose concentration medium with 25 \% PEG 4000. In B. napus, induction medium supplemented with PEG generated microspore derived embryos at low temperatures $\left(4,15\right.$, or $\left.18^{\circ} \mathrm{C}\right)$, suggesting that PEG treatment could replace the thermal stress of $32^{\circ} \mathrm{C}$ for 2 days in the induction of embryogenesis. PEG treatment had other advantages: the embryos were morphologically similar to zygotic embryos and conversion into plants and chromosome doubling (without colchicine) were increased (Ferrie and Keller 2007).

Additional stressing factors denote important possibilities to be considered. The $\mathrm{pH}$ role in cell and tissue culture is widely recognized (George et al. 2008), but the influence in microspore culture has been poorly studied. Yuan et al. (2012) reported an increment on embryogenesis when $\mathrm{pH}$ was increased from 5.8 to 6.4 in recalcitrant B. oleracea genotypes. The combination of a high $\mathrm{pH}$ value and the addition of $2-(\mathrm{N}$-Morpholino) ethanesulfonic acid (MES) and the gum arabic improved embryo yield up to thirty-fivefold in very low responsive genotypes. The effect of oligosaccharides was assessed by Lemonnier-Le Penhuizic et al. (2001) in B. oleracea. 30 min treatment of $34 \mu \mathrm{M}$ of carrageenan oligosaccharides increased two- to three-fold embryogenesis in combination to heat stress. Further studies evaluating the effect of such stresses would make possible the application of new methods in oilseed rape microspore culture. 


\subsection{Induced diploidization}

A generally low frequency of spontaneous diploidization has been found in microspore cultures of oilseed rape. The application of colchicine or alternative inhibitors of mitotic spindle tubers like pronamide and APM to freshly isolated microspores during the heat stress treatment for a period of one to three days led to induced diploidization rates of 70 to 90 \% (for a review see Ferrie and Möllers 2011). To improve the efficiency of the DH technology in oilseed rape breeding programs, it is desirable to obtain reproducible diploidization rates of $90 \%$ and higher. Increasing the inhibitor concentration did not enhance diploidization, and extending the treatment period beyond 72 hours increased frequency of tetraploid plantlets. Colchicine has also been shown to enhance microspore embryogenesis in B. napus and other species. As little as $25 \mu \mathrm{M}$ or $10 \mathrm{mg} / \mathrm{L}$ colchicine is effective in inducing diploidization in microspores and in improving embryogenesis (Iqbal et al. 1994; Zhao et al. 1996a). It remained an open question, if even lower concentrations of colchicine than $25 \mu \mathrm{M}$ are effective in diploidization and if the colchicine can be left in the medium without a washing step and without negative effects on microspore embryogenesis. Zhao et al. (1996) showed that washing steps to eliminate colchicine reduced embryo yield since cell injury and loss of induced cells is increased, whereas the dilution of the microspore solution to reduce colchicine concentration enhanced embryogenesis. Therefore, the addition of $10 \mathrm{mg} / \mathrm{l}$ of colchicine combined with a dilution of the microspore culture after two days avoiding centrifugation may lead to an improved embryo yield and enhanced diploidization frequency, as previously suggested by Zhao et al. (1996b).

A combined application of different microtubule inhibitors, also in combination with physical treatments (high and low temperature, pressure, electric current) has not shown any synergistic effect on the diploidization of maize root tips (Häntzschel and Weber 2010). Also, no synergistic effect of a combination of colchicine, APM and pronamide on diploidization of 8 different crosses of Brassica napus microspores was reported by Klutschewski (2013). Addition of the solvent dimethyl sulfoxide (DMSO) in concentration of $1 \%$ to the mannitol pretreatment medium resulted in an enhanced production of embryos and green plants in anther culture of Hordeum vulgare L. and Triticum aestivum L. (Echávarri and Cistué 2016). In contrast, addition of DMSO to the colchicine induction medium of Brassica napus did not lead to an enhanced 
diploidization rate (Klutschewski 2013). Another aspect is that pronamide, APM and oryzalin have a much lower toxicity to mammals compared to colchicine (Häntzschel and Weber 2010).

\subsection{Direct embryo to plant conversion}

Rapid and highly efficient conversion of microspore derived embryos to plants is the most desirable practical result for the production of double haploid populations. The ultimate goal would be that the embryos could be directly transferred to soil without any subculture on solid medium. However, direct embryo to plant conversion is genotype dependent and contrasting results under similar culture conditions were observed, which requires further improvement of the system (Ferrie and Möllers 2011).

A feature that has proved to be of great significance for direct embryo to plant conversion is the appropriate formation and maintenance of the shoot apical meristem (Stasolla et al. 2008). Results of Ferrie et al. (2005) indicated that the addition of Brassinolide not only increased the production of microspore derived embryos but also enhanced direct embryo to plant conversion. This effect is correlated with the brassinolide-related enhanced expression of BnSTM and BnCLV1 genes, which are markers for the shoot apical meristem formation (Belmonte et al. 2010; Belmonte et al. 2011). In agreement, the use of brassinazole, an inhibitor of brassinolide biosynthesis, caused the development of aberrant meristems and plant regeneration failed. Overexpression of AtDWF4, a brassinolide biosynthetic gene, resulted in overcoming the inhibitory effects on seedling germination of exogenous applied ABA (Divi and Krishna 2010). Belmonte et al. (2011) suggested that the correct embryo and apical meristem formation is related to the brassinolide capacity to modify purine and pyrimidine metabolism, since their role in the biosynthesis of polysaccharides, cofactors and cytokinins has been reported (Boldt and Zrenner 2003; Stasolla et al. 2003; Zrenner et al. 2006). Purine and pyrimidine biosynthesis is also correlated to ascorbate addition (Stasolla et al. 2001), which is needed for meristem reactivation during early phases of germination (Stasolla and Yeung 2006; Stasolla and Yeung 2007; Stasolla 2010). Brassinolide increases the recycling of ascorbate from oxidized forms (Belmonte et al. 2010) and exogenous application of BSO (DL-buthionine-[S,R]- sulphoximine) enhances 
ascorbate biosynthesis (Stasolla et al. 2008). Further research is required to more precisely identify the optimum concentration and microspore derived embryo developmental stage to achieve efficient embryo to plant conversion.

It is not known if the retarded shoot formation of embryos may be an effect related to primary seed dormancy, which is observed also in Brassica species (Fei et al. 2007). Different types of seed treatments have shown to improve germination. Osmopriming of oilseed rape seeds has notably improved germination performance by modulating several cellular processes prior to germination (Kubala et al. 2015). The use of polyethylene glycol 6000 as a seed pre-treatment accelerated germination in B. napus (Pace et al. 2012) and other member of the Brassicacea family (e. g. Jett et al. 1996; Gallardo et al. 2001).

Although the ability of microspore derived embryos to convert directly to plantlets is genotype depended, cold treatment significantly increased the direct embryo to plant conversion. Several studies have shown that direct plant regeneration can be dramatically improved by the cold treatment of embryos for a period of 3 to 28 days at $1^{\circ} \mathrm{C}-10^{\circ} \mathrm{C}$, while higher temperatures induced undesired secondary embryogenesis. (e. g. Coventry et al. 1988; Cegielska-Taras et al. 2002; Zhang et al. 2006). The influence of temperature and photoperiod on 13 oilseed rape genotypes was further assessed by Klutschewski (2013), who observed that the positive influence of cold treatment on direct embryo to plant conversion is improved when embryos are incubated under continuous darkness, suggesting an important role of photoperiod on direct embryo to plant conversion. Direct embryo to plant conversion enhancement by cold-treatment may arise from the fact that low temperatures induce cell dehydration (Chinnusamy et al. 2007), which is a fundamental step for embryo maturation (Fei et al. 2007). Accordingly, plantlet regeneration has been improved applying desiccation treatments (e. g. Kott and Beversdorf 1990; Zhang et al. 2006; Haddadi et al. 2008; Prem et al. 2008) or promoting embryo desiccation by ABA as a treatment prior to direct embryo to plant conversion induction (Hansen 2000). Very early research has shown that in Brassicas, ABA treatment increased desiccation tolerance of microspore derived embryos (Senaratna et al. 1991) and viability of embryos is enlarged (Takahata et al. 1993), suggesting their possible employment on the development of Brassica artificial seeds, that until now has been unsuccessful. 
Changes in the nutrient content of the induction media has also led to a better performance on direct embryo to plant conversion. Tian et al. (2004) showed that plantlets derived from MS media with Ca2+ concentration increased to $900 \mathrm{mg} / \mathrm{l}$ and half the micro and macronutrient content showed a similar morphology as zygotic seedlings, and plant regeneration was improved. Calcium has shown to increase seed germination of several angiosperm species, and even to alleviate the inhibition of germination by acid and salinity stresses (Tobe et al. 2004; Shaikh et al. 2007; Liu et al. 2011). Recent evidence indicate that the increase in cytosolic $\mathrm{Ca}^{2+}$ concentration neutralizes the negative effect of ABA, promoting seed germination in A. thaliana (Kong et al. 2015) while an excess of calcium is toxic and prevents seed germination (White and Broadley 2003). Oilseed rape is also known to have a comparatively high demand for the micronutrient boron (Zhang et al. 2014 and references therein). Although the NLN-medium contains with $10 \mathrm{mg} / \mathrm{l}(162 \mu \mathrm{M})$ relatively high concentrations of $\mathrm{H}_{3} \mathrm{BO}_{3}$, Sotiropoulos and Dimassi (2004) reported that shoot regeneration and shoot length was significantly enhanced in kiwifruit shoot cultures on medium with a twenty-fold higher boron concentration $(2 \mathrm{mM})$. However, the effect of increased $\mathrm{H}_{3} \mathrm{BO}_{3}$ concentration alone or in combination $\mathrm{CaCl}_{2}$ on the direct embryo to plant conversion has not yet been investigated in Brassica napus.

\subsection{Inheritance of microspore embryogenic potential, diploidization and direct embryo to plant conversion}

To identify genes involved in the microspore embryogenic pathway two different ways are followed. One way aims at investigating the inheritance of the genes determining the microspore embryogenic potential, and the other at comparing gene expression profiles of induced and non-induced microspores of genotypes with contrasting embryogenic response (Ferrie and Möllers 2011). In the first approach heterozygous F1 plants derived from crosses between two different genotypes are used to develop segregating recombinant inbred line (RIL) or doubled haploid (DH) populations, which are then used to map QTL for microspore embryogenic potential and to study distorted marker segregation. Genomic regions with molecular markers significantly deviating from the expected 1:1 segregation are thought to be linked to genes involved either in microspore embryogenesis, diploidization efficiency or direct embryo to plant 
conversion. In genetically characterized DH populations only genotypes are considered that finally regenerate to diploid fertile plantlets. Gamete genotypes that are not viable or embryogenic, which do not become diploid, and which do not readily form shoots are usually discarded. Hence, at each of those four steps skewed allele segregation may occur. Furthermore, skewed allele segregation may take place during the early stage of meiosis. This can only be detected if the $\mathrm{BC}_{1}$ plant generation is analyzed for marker allele segregation. Last, but not least, significantly distorted marker segregation can occur by chance. In the second approach, microspores from homozygous lines are cultivated under embryogenesis inducing $\left(32^{\circ} \mathrm{C}\right)$ and non-inducing conditions $\left(20^{\circ} \mathrm{C}\right)$ and differences in gene expression levels is used to identify candidate genes. Previous progress in comparing results from different populations was hampered by the lack of sequence informative markers and genome sequences (for a review see Ferrie and Möllers 2011). The availability of important Brassica genome sequences and of a large number of sequence informative SNP-markers now allows the comparison of physical positions of QTL and regions with distorted marker segregation across different populations.

To identify genomic regions that carry genetic factors controlling embryogenic potential of isolated microspores of rapeseed, Ecke et al. (2015) analyzed marker segregations in a segregating population of haploid microspore derived embryos and a $\mathrm{BC}_{1}$ population from a cross between the winter oilseed rape cultivar Express 617 and the resynthesized line RS239. After map construction 230 of 481 markers (48\%) showed significantly distorted marker segregations, whereas this value, was with only 26 of the markers (12\%) much lower in the $\mathrm{BC}_{1}$. In total, 34 regions with skewed segregations were identified, which were distributed over all linkage groups with the exception of A03 and C04. Regions with multiple distorted markers and large Chi2 values and no corresponding region with distorted segregation in the $\mathrm{BC}_{1}$ were detected on linkage groups A02, A05, A07, A09, C02, C03 and C06. Intervarietal substitution lines from the same cross with Express 617 as recurrent parent, which carried donor segments covering genomic regions that had shown skewed segregations were used to characterize their microspore embryogenic potential. Seven lines were identified that showed a 4.1 to 40.4 fold increase in the microspore embryogenic potential in comparison to the low embryogenic parent Express 617. Those lines carried between one and eight donor segments; they may be useful in further studies to narrow the 
genomic regions involved in embryogenesis and to finally identify relevant genes. In continuation of this study, Kampouridis et al. (2016a) analyzed marker segregation in populations of doubled haploid plantlets, of haploid and doubled haploid microsporederived embryos, and of $\mathrm{BC}_{1}$ plants, from the same cross, with the intention to identify regions showing distorted marker segregation specifically caused by genotypic differences in direct embryo to plant conversion. Among 26 regions with skewed marker segregation, only four regions on linkage groups C05, C07/C07a and two on C08 showed skewed segregations only in the DEPC population. Furthermore, by comparing marker segregation between segregating populations of haploid and colchicine induced diploid microspore derived embryos and of $\mathrm{BC}_{1}$ plants from the same cross, Kampouridis et al. (2016b) identified genomic regions on linkage groups A03, A06, A07, A08, C01, C04 and C08 that carried genetic factors controlling the colchicine induced diploidization rate of isolated microspores. In a Brassica rapa doubled haploid population derived from the cross of the highly embryogenic cultivar Ho Mei and the low responding cultivar CR-Seiga, Kitashiba et al. (2016) identified physical regions with strongly skewed marker segregations on A05, A08 and A09 with the Ho Mei alleles being predominant. The most pronounced distorted segregation was found on A08. These three regions showed the expected Mendelian segregation ratio in a F2 population, indicating that distorted segregations are not caused by differences in male gametogenesis but by differences in microspore embryogenesis. Analyzing a BC3F1 population from the same cross, it was shown that the region on A08 had the strongest effect on embryo yield from microspores and an additive effect was observed in combination with the region of A05. In a related study in barley Bélanger et al. (2016) used a pooled genotyping-by-sequencing approach to estimate allelic frequencies and to examine segregation distortion in 12 segregating populations derived from androgenesis. Applying a large number of SNP markers, they determined that on the average $28.9 \%$ of the markers showed distorted segregation and that $25.3 \%$ of the genetic map consisted of regions with distorted segregation. Across the 12 populations, 36 different distorted segregation peaks could be distinguished and 27 out of these 36 were observed in only one population. This reinforces the impression that the trait microspore embryogenesis is a polygenic trait and that dependent on the genotypes, different genomic regions may cause distorted segregation in different populations. If segregations are found to be significantly skewed, they may nevertheless occur by chance. 


\subsection{Identification of candidate genes for microspore culture}

response

Stress induced plant male germ cell reprogramming involves dramatic changes in the expression of a large number of genes which can only be explained by epigenetic mechanisms. Changes in DNA methylation, chromatin dynamics and small RNAs play an essential role in the regulation of stress-responsive gene expression in plant male germ cells (for a review see Chen et al. 2016). In undifferentiated vacuolated microspores and in microspores reprogrammed to embryogenesis of B. napus, Solis et al. (2012) found a significantly lower DNA methylation and transcript abundance of DNA methyltransferase I (BrMET1a) compared to differentiated mature pollen as well as globular and cotyledonary microspore derived embryos, supporting earlier results showing a higher general gene expression level in undifferentiated cells. Li et al. (2012) also cloned a putative DNA methyltransferase from Brassica rapa (BcMF22), which was preferentially expressed during pollen development starting from pollen mother cell until mature pollen.

By comparing differentially expressed genes in induced embryogenic and nonembryogenic microspores Malik et al. (2007) were able to identify a specific number of genes that are expressed in haploid and zygotic embryos, but not during pollen development (for a review see Soriano et al. 2013). Among those genes were FUSCA3, LEAFY COTYLEDON1 (LEC1), LEC2, BABY BOOM (BBM), two WUSCHEL-related homeobox (WOX) genes, WOX2 and WOX9, and ABSCISIC ACID INSENSITIVE3. However, only the expression of LEC2 was useful to distinguish between embryogenic and non-embryogenic cultures after 3 days in culture. Analyzing genomic regions with skewed segregation, Kitashiba et al. (2016) were not able to collocate the position of the candidate genes BBM1 (Boutilier et al. 2002), LEC1 (Meinke et al. 1994), ABI3 (Parcy et al. 1994) and CYP81F (Malik et al. 2007) with their three identified regions with skewed segregation (see above). Furthermore, the four candidate genes were located on A10, A03, A07 and A10, respectively, and those loci did not show distorted segregation in the doubled haploid population Ho Mei x CR-Seiga. Together, this casts some doubt on the importance of those genes in microspore embryogenesis. Kitashiba et al. (2016) were able to identify the locus Bra029631 at 110kb distance to the Br110 locus on A05. 
Bra029631 is homolog to Bnm4D-84, a gene which is highly expressed in globular to torpedo stage embryos (Tsuwamoto et al. 2007). In addition, several other candidate genes, like CLE (CLAVATA/ESR-RELATED)-like gene (Bra016537), MEE67 (MATERNAL EFFECT EMBRYO ARREST 67) (Bra016560) and HSP70B (70 kDa class heat-shock protein) (Bra016644), were found to be located adjacent to the Br071-5c locus on A08 within a distance of $11 \mathrm{up}$ to $486 \mathrm{~kb}$ (Kitashiba et al. 2016). The BBM gene belongs to the AP2/ERF-family (Boutilier et al. 2002; Lv et al. 2016). The ethylene response transcription factor (ERF) family proteins play an important role in regulating a variety of stress responses in plants (Lv et al. 2016). In B. napus, the expression of BnERF2.4 was induced by submergence and the overexpression of BnERF2.4 in Arabidopsis increased the level of tolerance to submergence and oxidative stress ( $\mathrm{Lv}$ et al. 2016). Interestingly, isolated microspore cultures in liquid medium may also suffer from submergence stress which, in addition to the heat stress may cause enhanced ethylene biosynthesis. This matches well with the observation that the above mentioned addition of cobalt chloride to the culture medium improves microspore embryogenesis (Leroux et al. 2009).

Although direct embryo to plant conversion is still a bottleneck in efficient application of DH technology, little is known about the genetic and exogenous regulation of this developmental step in microspore derived embryos. The establishment of a shoot apical meristem is an important event in embryogenesis, and the maintenance of its structure and functionality seems to be a major factor influencing direct embryo to plant conversion (Belmonte et al. 2006; Stasolla et al. 2008). This process is regulated by a number of genes (Elhiti et al. 2010). Overexpression of the gene SHOOTMERISTEMLESS (STM), which enhances microspore embryogenesis, has shown to modulate the expression of several other genes involved in the establishment of cell fates in embryo cultures of Brassica species and A. thaliana, for instance, ZWILLE (ZLL), ARGONAUTE1 (AG01), CLAVATA (CLV), WUSCHEL (WUS), WUSCHEL RELATED HOMEOBOX (WOX), CUP-SHAPED COTYLEDON (CUC) and SCARECROW-LIKE genes (SCL) (Tsuwamoto and Takahata 2008; Stasolla et al. 2008; Elhiti et al. 2010; Elhiti et al. 2012; Elhiti et al. 2013). However, their direct and indirect influence of direct embryo to plant conversion is still unknown. 


\section{Chapter 3}

Phenotypic variation and QTL mapping of microspore embryogenic potential and direct embryo to plant conversion in the DH population DH4079 $\mathrm{x}$ Express (Brassica napus L.) 


\subsection{Abstract}

Microspore culture is a very powerful technique in breeding of oilseed rape for the rapid and efficient generation of completely homozygous doubled haploid (DH) lines. Despite the progress achieved in optimizing tissue culture protocols, tremendous differences remain among $B$. napus genotypes in their embryogenic response and the genetic basis of those differences is still largely unknown. Furthermore, direct embryo to plant conversion efficiency is a hindrance to tissue culture success. The objective of this work was to study the genetic variation and inheritance of the embryogenic potential and direct embryo to plant conversion in a DH population of the cross DH4079 $\mathrm{x}$ Express 617, and to determine genomic regions and putative candidate genes responsible for those traits. Survival of embryos, root regeneration and secondary embryogenesis were also evaluated. The parental lines were selected due to their contrasting response in microspore embryogenic potential and direct embryo to shoot conversion. First, F1-plants of the cross DH4079 x Express 617 were used to generate a DH population of 204 lines. Then, those DH lines were seed propagated and used again as the source of microspores, which were cultured following a standard protocol. The number of microspores and microspore embryogenic potential, defined as the percentage of microspores developing embryos, were recorded. Embryos were transferred to Gamborg's B5 medium supplemented with $0.1 \mathrm{mg} / \mathrm{L}$ gibberellic acid and following a cold treatment, the survival, secondary embryogenesis, direct embryo to plant conversion and root regeneration were scored. Experiments were repeated five times and mean values obtained from 81 to 98 lines were used for QTL mapping based on an Illumina Infinium Brassica 60K SNP molecular linkage map. Large and significant genetic differences among the genotypes were found for all traits. The microspore density of cultures based on 16 buds per experiment varied in the DH population from 58,900 to 148,700 per $\mathrm{ml}$ as a mean over 5 experiments from . Microspore embryogenic potential ranged from 0 to $3.6 \%$. Embryo survival varied from $26 \%$ to $99 \%$, and direct embryo to plant conversion was found to range from $13 \%$ to $85 \%$. Root regeneration varied from $14 \%$ to $95 \%$, and secondary embryogenesis occurred in $4 \%$ up to $91 \%$ of the embryos. Heritabilities ranged from $66 \%$ for microspore density to $86 \%$ for microspore embryogenic potential. A positive correlation between survival of embryos, direct embryo to plant conversion, root regeneration and secondary embryogenesis was 
observed $\left(\mathrm{r}_{\mathrm{s}}=0.37^{* *}\right.$ to $\left.0.78^{* *}\right)$. A linkage map based on 1,414 SNP markers was developed from which $49 \%$ exhibited distorted segregation clustered over all linkage groups, except for chromosome A04. Together 10 QTL were mapped on linkage groups A01, A02, A05, A10, C04 and C06. For microspore embryogenic potential three QTL were detected, which together explained $31.7 \%$ of the phenotypic variance observed in the DH population. One major QTL $\left(R^{2} \geq 25 \%\right)$ was identified for the trait survival of microspore derived embryos explaining $26.1 \%$ of the phenotypic variance. Two QTL each were detected for secondary embryogenesis and direct embryo to plant conversion, which explained $34.8 \%$ and $35 \%$ of the phenotypic variance, respectively. For root regeneration, two QTL were determined which explained $34.5 \%$ of the phenotypic variance. Overlapping confidence intervals of QTL in linkage group A05 indicated an important region responsible for microspore embryogenic potential. BLAST-analysis revealed the presence of nineteen candidate genes within the $95 \%$ confidence interval of QTL. Among those, CLAVATA3/EMBRYO SURROUNDING REGION-RELATED PEPTIDE 1, CLAVATA3/EMBRYO SURROUNDING REGION-RELATED PEPTIDE 25, WUSCHEL RELATED HOMEOBOX 1, WUSCHEL RELATED HOMEOBOX 2, WUSCHEL RELATED HOMEOBOX 5, CUP-SHAPED COTYLEDON 3, SCARECROW and NOAPICAL-MERISTEM were previously reported candidate genes related to microspore embryogenic potential, secondary embryogenesis and direct embryo to plant conversion. 


\subsection{Introduction}

Brassica napus has become an important model plant to study functional genomics underlying androgenesis, because Arabidopsis thaliana belongs to the non-responsive species in microspore embryogenesis (Hosp et al. 2007). In this regard, it is of great importance to complement the knowledge already generated with QTL analysis that integrates phenotypic and genotypic information in order to identify genomic regions involved in the main phases and constraints of microspore culture: diploidization, microspore embryogenesis and direct embryo to plant conversion.

The first successful research about microspore embryogenesis of oilseed rape was published by Lichter (1982). Since then, numerous authors have developed protocols to enhance androgenesis success of Brassica napus focusing on pre-culture treatments, media composition, culture density, hormone and antimitotic induction and temperature stress. Furthermore, some studies have aimed at improving direct embryo to plant conversion (for a review see Ferrie and Möllers 2011; Soriano et al. 2013; Rahman and Michalak de Jiménez 2016). From those analyses, it has been possible to identify numerous factors influencing the androgenesis process and plant regeneration of microspore derived embryos. Despite such progress achieved in optimizing tissue culture protocols, it also became clear that success in microspore culture is genotype dependent, leading to a highly heterogenic response in embryo yield and plant regeneration under comparable culture conditions.

In Brassica napus, few approaches have studied genetic causes of differences in the capacity of microspores to generate embryos and direct embryo to plant conversion. Since it is assumed that microspore derived embryos are generated from a random sample of gametes from a heterozygous plant, a Mendelian 1:1 segregation ratio is expected for a monogenic trait. However, already early work on isozyme genetic markers detected distorted segregation in DH populations (Foisset et al. 1993). Further studies in oilseed rape confirmed that distorted segregation is a persistent phenomenon in F1-plant derived doubled haploid populations (Cloutier et al. 1995; Tanhuanpää et al. 1994; Zhang et al. 2003). Results from different crosses between responsive and nonresponsive genotypes revealed segregation distortions at diverse loci located in various 
linkage groups, suggesting a dissimilar origin of selective pressures during in vitro culture and the importance of the genetic background for microspore culture and plant regeneration success. Ono and Takahata (2000) reported high heritability values of shoot regeneration from seedling derived cotyledonary explants, as well as a predominant contribution of additive effects to phenotypic variance in a $7 \mathrm{x} 7$ diallel cross. However, dominant effects were also significant. In agreement with these results, heritability for microspore embryogenic ability, determined as number of embryos regenerated from a certain number of microspores, in a $4 \times 4$ Brassica napus diallel cross was high and additive effects were predominant (Zhang and Takahata 2001). A first effort to identify putative genes associated with microspore embryogenic potential was undertaken by Cloutier et al. (1995), who recognized 5 loci on linkage groups 01 and 18 possibly involved in androgenesis, based on a comparative segregation analysis using a $F_{2}$ population and two $F_{1}$ microspore-derived populations from the same cross between the microspore culture-responsive parent Topas and the non-responsive parent Westar. Further research on gene expression led to the identification of several candidate genes that were up-regulated in response to acquisition of microspore embryogenic potential and highly expressed during zygotic embryo development (Joosen et al. 2007; Malik et al. 2007; Stasolla et al. 2008; Elhiti et al. 2010; Elhiti et al. 2013).

The increasing understanding of the genetic factors involved in the reprogramming of microspore development into embryos and its further direct conversion into plants could be enormously advantageous for the early diagnosis of responsive and not responsive genotypes. Furthermore, the better understanding of the genetic regulation mechanisms of androgenesis is of great importance to establish strategies to overcome the poor microspore and embryo responsiveness in recalcitrant genotypes and species (Boutilier et al. 2005; Hosp et al. 2007).

In this context, the objectives of the present research were to develop a microsporederived DH population from the cross DH4079 x Express 617, to study the genetic variation and inheritance of the microspore embryogenic potential and direct embryo to plant conversion in a DH population, and to map QTL and evaluate the possible presence of candidate genes for those traits. Among the genotypes known, the Swedish spring cultivar Topas has been extensively studied for its excellent microspore 
embryogenic potential and the derived line DH4079 showed an outstanding embryo production of many thousand embryos per experiment, which has made DH4079 a standard in many investigations (Boutilier et al. 2002; Cloutier et al. 1995; Ferrie et al. 2005; Ferrie and Keller 2007; Malik and Krochko 2009; Malik et al. 2007). In contrast, a very low embryo yield is obtained from inbred line 617 of winter oilseed rape cultivar Express, in the range of nil up to 50 embryos per experiment under comparable conditions (Klutschewski 2013; Ecke et al. 2015). Moreover, a good direct embryo to plant conversion was found for Express 617, whereas the response was only moderate for DH4079 (Klutschewski 2013). 


\subsection{Materials and Methods}

\subsubsection{Development of the DH Population}

Seeds of the inbred line 617 of the winter oilseed rape cultivar Express were obtained from Norddeutsche Pflanzenzucht Hans-Georg Lembke KG (www.npz.de). Seeds of DH4079 (Ferrie 2003) were provided by Alison Ferrie (NRC Saskatoon, Canada). An F1 seed derived from the cross between Express 617 and DH4079 was germinated under aseptic conditions on Murashige and Skoog (1962) medium and the plantlet was in vitro clonally propagated. Cloned F1-plants were transferred to soil (Fruhstorfer T25-Erde) in multipot trays. After acclimatization, plants were cultivated in the greenhouse for 4 weeks. Subsequent to this period, plants were transferred to $13 \mathrm{~cm}$ pots and vernalized for 8 weeks in a cold chamber $\left(4^{\circ} \mathrm{C}, 8 \mathrm{~h}\right.$ light). After that, plants were again cultivated in the greenhouse. Upon bolting, plants were transferred to a growth chamber $\left(12 / 6{ }^{\circ} \mathrm{C}\right.$ light/dark, 16/8 h light/dark) and were used as microspore donors. The growth chamber was equipped with Sodium-vapor lamps (SON-T Agro Philips Master Green Power CGT 400 watt, light intensity of $400 \mu \mathrm{mol} \mathrm{m} \mathrm{m}^{-2} \mathrm{~s}^{-1}$ ). Donor plants were watered three times per week and fertilized once a week with N:P:K (15:11:15).

For microspore isolation, buds with 2.5 to $3.5 \mathrm{~mm}$ length with microspores at the late uninucleate stage were collected and treated as previously described by Möllers et al. (1994) with minor modifications. 16 buds were surface sterilized with $1 \%$ calcium hypochlorite solution and Tween-20 in constant stirring for 5 minutes. Buds were rinsed 3 times with autoclaved deionized water and transferred to a nylon sieve $(50 \mu \mathrm{m}$, $5 \mathrm{~cm}$ in diameter) in a Petri dish (92 x $16 \mathrm{~mm}$, Sarstedt AG and Co., Nürnbrecht, Germany) containing $5 \mathrm{ml}$ of liquid filter sterilized NLN13-medium (Lichter 1982; www.duchefa-biochemie.com, catalog no. N0252). Buds were squeezed in the sieve with the flat end of a pistil to release the microspores. The sieve was then rinsed with $7 \mathrm{ml}$ of NLN13-medium. The resultant $12 \mathrm{ml}$ microspore suspension was transferred to a tube, which was then centrifuged for 5 minutes at 1000 RPM. The supernatant was carefully decanted and the pellet was washed twice with NLN13-medium. The final microspore pellet was resuspended in $12 \mathrm{ml}$ NLN13-medium and transferred to a Petri dish (92 x $16 \mathrm{~mm}) .600 \mu \mathrm{l}$ of a colchicine stock solution $(2 \mathrm{mg} / \mathrm{ml}$ ) were added to obtain a final 
concentration of $100 \mathrm{mg} / \mathrm{L}$ and Petri dishes were sealed twice with Parafilm. Petri dishes were incubated $48 \mathrm{~h}$ at $32{ }^{\circ} \mathrm{C}$ in the dark. Then, the microspore suspension was transferred to a tube and centrifuged as described above. The supernatant was discarded and the pellet resuspended in $12 \mathrm{ml}$ NLN was transferred to a new Petri dish. Cultures were incubated 8 days at $28{ }^{\circ} \mathrm{C}$ in the dark and then transferred to an orbital shaker $(40 \mathrm{rpm})$ in a culture room $\left(22^{\circ} \mathrm{C}, 12 / 12 \mathrm{~h}\right.$ light/dark, Osram L Lumilux daylight $58 \mathrm{~W}$, with a light intensity of $70 \mu \mathrm{mol} \mathrm{m} \mathrm{m}^{-2} \mathrm{~s}^{-1}$ ), after adding $2 \mathrm{ml}$ of fresh NLN13medium.

Twenty-one days after culture initiation, microspore derived embryos were transferred to liquid medium at a density of 30 embryos/12 ml NLN13-medium in Petri dishes (92 x $16 \mathrm{~mm}$ ), which were incubated for 7 days on a shaker (40 rpm) in the same culture room and under the same conditions as described above. Well differentiated microspore derived embryos at the late cotyledonary stage with a size of 0.7 to $1.0 \mathrm{~cm}$ in length were transferred to plastic boxes $(10.5 \times 8 \times 5 \mathrm{~cm}$, Volume $0.24 \mathrm{~L}$, Huhtamaki GmbH and Co.KG, Alf, Germany) containing B5-medium complemented with $0.1 \mathrm{mg} / \mathrm{L}$ gibberellic acid and solidified with 0.9 \% Plant Agar (Gamborg et al. 1968; Plant Agar, C.E. Roeper $\mathrm{GmbH}$, Germany). Eight microspore derived embryos were placed in each plastic box, which were then incubated for 10 days at $2{ }^{\circ} \mathrm{C}$ in the dark (Cegielska-Taras et al. 2002). After that period, boxes with embryos were transferred to shelves in the same culture room and under the same light and temperature conditions as described above. Direct embryo to plant conversion was scored once a week for up to 10 weeks after transfer to B5-medium. The span (weeks) that occurred after the transfer of embryos to B5 medium until the appearance of the shoot was recorded as "Readiness". Regenerated plantlets were subcultured and finally transferred to soil and acclimatized as previously described.

Leaf samples were taken to confirm that diploidization had occurred (Möllers et al. 1994). Therefore, $1 \mathrm{~cm}^{2}$ leaf tissue samples were dissected and carefully chopped with a sharp blade. $1 \mathrm{ml}$ of staining buffer (fluorochrome solution 4'6-diamidino-2phenylindole solution, DAPI) was added and the resulting suspension was filtered by means of a nylon sieve $(40 \mu \mathrm{m})$ to remove plant tissue debris. The nucleus containing suspension was analyzed with a Partec Cell Analyser CA-II flow cytometer. Confirmed doubled haploid plants were vernalized for 8 weeks as described above. Then, plants 
were transferred to the greenhouse and at begin of flowering, inflorescences were covered with microspore crisp bags to secure self-pollination.

\subsubsection{Characterization of the DH Population}

In total 204 doubled haploid plants were regenerated fromF1-plants of the cross DH4079 x Express 617 from 4 independent microspore cultures. Consequently, 4 subpopulations of 56, 53, 48 and 47 DH lines were generated. Following self-pollination, seeds harvested from those lines, parental genotypes and F1 were sown in batches of 20 lines a two seeds per genotype in the greenhouse. Plants were grown until the 3 rd to 4th leaf stage and then they were vernalized for 8 weeks. Individual plants were then repotted to $13 \mathrm{~cm}$ pots using standardized soil (Fruhstorfer T25-Erde) and cultivated in the greenhouse. At bolting, plants were transferred to the growth chamber and cultivated under the same conditions as described above. At the begin of flowering, 2.5 to $3.5 \mathrm{~mm}$ long buds with microspores at the late uninucleate stage were collected and used for microspore isolation as previously described. Once the microspore pellet was obtained, it was resuspended in $12 \mathrm{ml}$ NLN13-medium containing $10 \mathrm{mg} / \mathrm{L}$ colchicine to induce diploidization. A drop of the microspore suspension was extracted and stored in

a $1 \mathrm{ml}$ microcentrifuge tube for a subsequent analysis of the microspore density (number of microspores/ml), which was accomplished using a Fuchs-Rosenthal counting chamber. The suspension was shifted to a Petri dish (92 $16 \mathrm{~mm})$ and it was sealed twice with Parafilm. Microspore preparations were incubated in the dark for 48 hours at $32{ }^{\circ} \mathrm{C}$. After this period, the microspore solution was transferred to a tube and centrifuged for $5 \mathrm{~min}$ as described before. The supernatant was discarded and the pellet was resuspended in $12 \mathrm{ml} \mathrm{NLN}$ in a Petri dish, which was incubated 8 days in the dark at $28{ }^{\circ} \mathrm{C}$. Subsequently, $2 \mathrm{ml}$ of fresh NLN13-medium were added and microspore cultures were transferred to an orbital shaker (40 rpm) in a culture room $\left(22{ }^{\circ} \mathrm{C}, 12 / 12\right.$ h light/dark, Osram L Lumilux daylight 58W, with a light intensity of $70 \mu \mathrm{mol} \mathrm{m}^{-2} \mathrm{~s}^{-1}$ ). 15 days after culture initiation, half of the developed microspore derived embryos were transferred to a new Petri dish containing $12 \mathrm{ml}$ NLN13-medium. For the remaining half of the embryos $10 \mathrm{ml}$ of NLN13-medium were carefully replaced by $10 \mathrm{ml}$ of fresh NLN13-medium. At day 20 after culture initiation, the number of regenerated embryos was recorded. In this way, microspore embryogenic potential was calculated as the percentage of microspores developing microspore derived embryos. 
At this stage, from each experiment 30 microspore derived embryos were transferred to each of three new Petri dishes with $12 \mathrm{ml}$ NLN13-medium. The cultures were incubated for approximately 7 days in the same culture room and under the same conditions as described above. Microspore derived embryos at late cotyledonary stage with a size of 0.7 to $1.0 \mathrm{~cm}$ in length were transferred to B5 medium supplemented with $0.1 \mathrm{mg} / \mathrm{L}$ gibberellic acid and solidified with 0.9 \% Agar (Plant Agar, C.E. Roeper GmbH, Germany). 8 microspore derived embryos were placed in each of the 7 plastic boxes per experiment and genotype (56 embryos/ experiment / genotype). Cold treatment of embryos and their subsequent transfer to a culture room were accomplished as described above. 6 weeks after transfer to B5-medium the traits survival, direct embryo to plant conversion, root regeneration and secondary embryogenesis of the microspore derived embryos were scored. The trait survival was determined as the percentage of microspore derived embryos that were alive after 6 weeks of being transferred to B5 medium. Root regeneration was calculated as the percentage of microspore derived embryos which developed roots from the hypocotyl. Direct embryo to plant conversion was determined as the proportion of microspore derived embryos that regenerated into plants, presenting both shoot and root. The trait secondary embryogenesis was scored as the percentage of microspore derived embryos that generated somatic embryos.

\subsubsection{Experimental design and Statistical Analysis}

To study the microspore density and the microspore embryogenic potential, five independent microspore cultures experiments were done per genotype, in which the number of microspores per $\mathrm{ml}$ of medium and percentage of microspores developing microspore derived embryos were scored. To evaluate the remaining traits, 56 microspore derived embryos derived from each of the previously mentioned experiments were cultured in plastic boxes, achieving a total of 7 replicates 7 boxes with 8 embryos each) per experiment and genotype to determine the traits survival, direct embryo to plant conversion, root regeneration and secondary embryogenesis. For the microspore culture experiments and the remaining traits, the following two ANOVA models were used:

(1) $Y_{i j}=\mu+g_{i}+g_{i} e_{j}$

(2) $\quad Y_{i j k}=\mu+g_{i}+e_{j}+g_{i} e_{j}+r_{k}: g_{i} e_{j}$ 
In model (1) $Y_{i j}$ is the trait value of the genotype ${ }_{\mathrm{i}}$ in the experiment $\mathrm{j}_{\mathrm{j}} \mu$ is the general mean, and $g_{i}$ and $e_{j}$ are the effects of the genotype and the experiment, respectively. $g_{i} e_{j}$ is the interaction between ${ }_{i}$ th genotype and ${ }_{j}$ th experiment. In model (2) $Y_{i j k}$ is the trait value of the genotype ${ }_{\mathrm{i}}$ in the experiment ${ }_{\mathrm{j}}$ and replicate (box) ${ }_{\mathrm{k}}, \mu$ is overall mean, $g_{i}$ and $e_{j}$ are effects of genotype $i$ and experiment $j$, respectively; $g_{i} e_{j}$ is the interaction between ${ }_{i}$ th genotype and ${ }_{j}$ th experiment, and $p_{k}: g_{i} e_{j}$ is the effect of the replicate $\mathrm{k}$ within the genotype i and the experiment $\mathrm{j}$.

The data were tested for outliers based on the Cook Distance using the "Influence.ME" package of R software version 3.2.2 (Nieuwenhuis et al. 2012; R Development Core Team 2016). To estimate variance components the PLABSTAT software was employed (Utz 2011). All factors were treated as random and broad-sense Heritability $\left(\mathrm{H}^{2}\right)$ was calculated as the following ratio (Hill et al. 1998):

$$
\begin{gathered}
\text { (1) } H^{2}=o^{2} g /\left(o_{g}^{2}+o^{2}{ }_{g e} / E\right) \\
\text { (2) } H^{2}=o^{2} g /\left({O^{2}}_{g}+{O^{2}}_{g e} / E+{O^{\prime}}_{\varepsilon} / E R\right)
\end{gathered}
$$

where $E$ is the number of experiments and $R$ the number of replicates. Spearman's rank correlation coefficients based on the mean values of each genotype were calculated between all traits. Correlations were computed using the "cor" function from the basic "stats" package of R software version 3.2.2 (R Development Core Team 2016). Results from the trait microspore embryogenic potential were square root transformed to achieve a better fit to normal distribution before statistical analysis.

\subsubsection{SNP Marker Analysis and Linkage Map Development}

$100 \mathrm{mg}$ of leaf tissue from young plants of each genotype of the DH population grown in the greenhouse were sampled in $2 \mathrm{ml}$ reaction tubes (Safe lock), which were immediately frozen in liquid nitrogen and stored at $-80^{\circ} \mathrm{C}$. DNA was isolated using the innuPREP Plant DNA Kit (Analytik Jena AG) and each sample was diluted to achieve a density of $50 \mathrm{ng} \mathrm{DNA} / \mu \mathrm{l}$ in a total volume of $20 \mu \mathrm{l}$. SNP analysis was performed by the company TraitGenetics GmbH, Gatersleben, based on the Illumina Infinium 60k Chip (Clarke et al. 2016). Samples from the parental lines DH4079 and Express 617 and the cross (F1) were also collected and analyzed. A linkage map was kindly provided by 
TraitGenetics GmbH (Gatersleben, Germany). From 52,157 SNP markers 22,714 resulted polymorphic. 3,143 markers were selected and a high-density genetic map was developed using the software JoinMap 4.1 (Stam 1993). The final linkage map covered 2,345.5 cM genome length with an average marker distance of $1.69 \mathrm{cM} .1,414$ markers distributed over the 19 linkage groups were used for the final framework map.

\subsubsection{QTL Analysis and Study of Distorted Marker Segregation}

Identification of quantitative trait loci (QTL) was performed using the software WinQTL Cartographer version 2.5 (Wang et al. 2012) and composite interval mapping (CIM) algorithm was employed. The linkage map developed by Trait Genetics GmbH was employed to determine QTL positions. Independent LOD significance thresholds $(\alpha=0.05)$ were estimated for each trait by 1000 permutation tests. Model 6 was employed and forward and backward stepwise regression method was used to set cofactors. The genome was scanned at $1 \mathrm{cM}$ intervals and the window size was set to 10 cM. $95 \%$ confidence intervals for QTL were determined by the one LOD score down method from the peak position. Names of QTL were established as a junction of the abbreviation of the trait name, the linkage group involved and the marker position on the linkage group.

Additive effects, as well as the percentage of phenotypic variance explained by a QTL, were also estimated by means of WinQTL Cartographer software version 2.5. Goodnessof-fit to the expected Mendelian segregation 1:1 (P $\leq 0.05)$ of SNP markers in the DH population was computed by a Chi-square test using the "chisq.test" function from the basic "stats" package of R software version 3.2.2.

\subsubsection{Synteny Analysis for Candidate Gene Localization}

QTL positions were studied and closely related SNP markers were characterized according to the maximum LOD score for the traits microspore embryogenic potential and direct embryo to plant conversion. $95 \%$ confidence intervals of related genomic regions previously identified were used to select the areas were putative candidate genes could be found. To achieve this, sequences of flanking SNP markers (Clarke et al. 2016) were used for BLAST analysis to identify physical positions of QTL using the $B$. 
oleracea Genome Database (Bolbase; http://www.ocri-genomics.org/bolbase/) and the B. rapa Genome Database (BRAD; http://brassicadb.org/brad/index.php). B. oleracea and B. rapa databases were consulted since the gene annotation is more comprehensive compared to B. napus reference genome (Chalhoub et al. 2014). Physical positions of genes reported in preceding studies associated with embryogenesis and microspore culture were compared to the positions of the QTL. In addition, a gene inquiry on $B$. oleracea and B. rapa databases was performed by applying the search tool for the following keywords: 'apical meristem', 'bilateral symmetry', 'cotyledon', 'embryogenesis', 'gametophytic', 'germination', 'gibberellin', 'microspore', 'regeneration'. Physical positions of the predicted genes resulting from such inquiry were also compared to the physical positions of the QTL presented in this manuscript. 


\subsection{Results}

\subsubsection{Phenotypic Analysis and Heritability}

The readiness with which the embryos derived from the F1-plants DH4079 x Express showed direct embryo to plant conversion was scored. Embryos started to form shoots after two weeks and shoot regeneration stopped after 9 weeks. The average shoot regeneration time was 4.9 weeks (Figure 3.1).

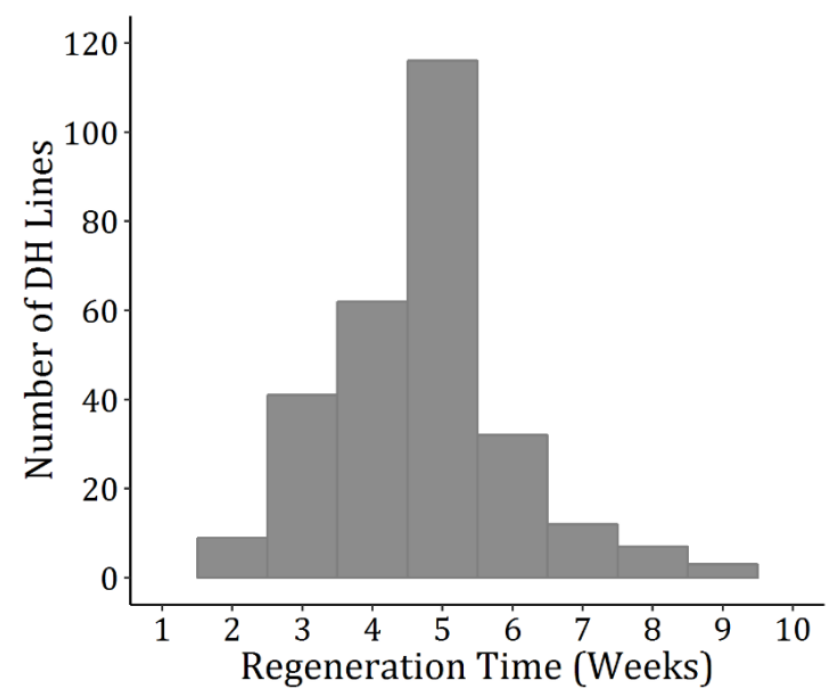

Figure 3.1 Frequency distribution of the direct embryo to plant conversion of the segregating microspore derived embryo population derived from the F1 DH4079 x Express 617.

A DH population of 204 lines was generated from the cross DH4079 x Express 617, from which 98 genotypes were phenotypically characterized for the trait density of microspores and 96 for the trait microspore embryogenic potential. Survival of embryos was assessed in $96 \mathrm{DH}$ lines and direct embryo to plant conversion in 85 genotypes, while root regeneration and secondary embryogenesis was studied in 82 and 86 lines, respectively. Analysis of variance revealed significant effects of the genotype on all studied traits (Table 3.1). Even though the effect of the genotype $\mathrm{x}$ experiment interaction was significant, the variance component values were smaller than the genotype effect. The effect of the factor experiment was only significant for the traits microspore density and embryo survival. Moderate to high broad-sense heritabilities for all traits ranged from 0.66 to 0.86 . 
Table 3.1 Variance components and heritabilities of the DH population.

\begin{tabular}{|c|c|c|c|c|c|c|}
\hline \multirow{2}{*}{ Trait } & \multirow{2}{*}{ DF } & \multicolumn{4}{|c|}{ Variance Components $^{d}$} & \multirow{2}{*}{$\begin{array}{c}\text { Heritability } \\
\left(H^{2}\right)\end{array}$} \\
\hline & & $O^{2} g$ & $O^{2} e$ & $o^{2} g e$ & $o^{2}{ }_{\varepsilon}$ & \\
\hline Microspore Density $(\mathrm{mic} / \mathrm{ml})^{a}$ & 97 & $230.6^{* *}$ & $48.5^{* *}$ & 603.6 & - & 0.66 \\
\hline Microspore Embryogenic Potential (\%) ${ }^{b}$ & 95 & $20.1^{* *}$ & 0.1 & 15.8 & - & 0.86 \\
\hline Embryo Survival (\%) & 95 & $206.4^{* *}$ & $7.8^{* *}$ & $432.5^{* *}$ & 287.3 & 0.69 \\
\hline DEPC $(\%)^{c}$ & 84 & $257.9 * *$ & 2.6 & $311.8 * *$ & 342.8 & 0.78 \\
\hline Root Regeneration (\%) & 81 & $319.9 * *$ & 2.9 & $270.8^{* *}$ & 374.2 & 0.83 \\
\hline Secondary Embryogenesis (\%) & 85 & $424.2 * *$ & 0.8 & $371.8^{* *}$ & 273.0 & 0.84 \\
\hline
\end{tabular}

a original values $/ 100$

${ }^{\mathrm{b}}$ square root transformed values $\times 100$

c DEPC = Direct Embryo to Plant Conversion

${ }^{\mathrm{d}} \mathbf{O}^{2} \mathrm{~g}=$ genetic variance, $\boldsymbol{O}^{2}{ }_{\mathrm{e}}=$ environmental variance, $\mathrm{O}^{2} \mathrm{ge}_{\mathrm{e}}=$ variance of genotype $\mathrm{x}$ environment interaction, $\boldsymbol{O}^{2}{ }_{\varepsilon}=$ residual error

** denotes significance at $\mathrm{P}<0.01$ 
Extremely contrasting culture responses were observed among the characterized lines of the DH population, as well as in the parental genotypes, not only for the studied traits but also in morphology, color and size (Figure 3.2).
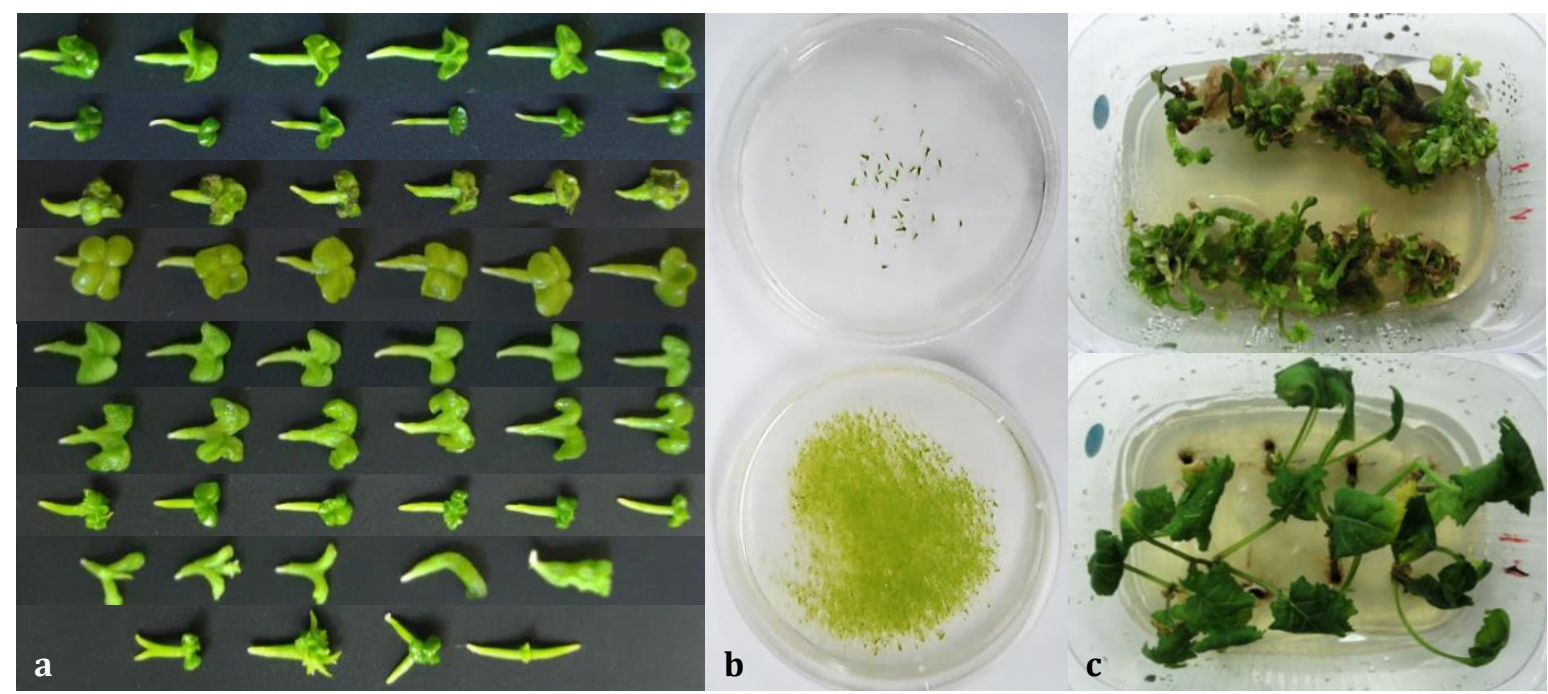

Figure 3.2 Highly different responses to microspore culture: a) morphological variation among genotypes of the DH population, b) quantity of microspore derived embryos produced by parental lines Express 617 (above) and DH4079 (below), and c) secondary embryogenesis response (above) and direct embryo to plant conversion (below).

The phenotypic traits showed weakly skewed and normally distributed residuals, except for microspore embryogenic potential that displayed a strongly right-skewed frequency distribution (Figure 3.3 and 3.4). The microspore density of cultures based on 16 buds per experiment varied in the DH population from 58,900 to 148,700 per $\mathrm{ml}$ as a mean over 5 experiments. Parental genotypes and the F1 did not differ significantly from each other (Table 3.2). The microspore embryogenic potential of the studied DH lines ranged from 0 to $3.6 \%$ with a mean of $0.2 \%$, meaning that 0 to 43,000 microspore derived embryos were developed as a mean over the experiments. Parental genotypes and F1 showed significant differences in their microspore embryogenic potential. The value for the microspore embryogenic potential of the parental line Express 617 was extremely low (0.003\%), no DH line with a significantly lower value was identified but four DH lines did not produce embryos. 16 out of 98 lines, showed a greater microspore embryogenic potential compared to the high responsive parent DH4079 (1.41\%). The 
F1 presented an intermediate value of $0.5 \%$. The extreme values of the highly responding $\mathrm{DH}$ lines compared to the parental genotype suggested transgressive segregation. The trait survival varied from $26 \%$ to $100 \%$ and displayed a mean of 77 $\%$; no significant differences were found among the parental genotypes and the F1 for the survival of embryos. Direct embryo to plant conversion ranged from $13 \%$ to $85 \%$ with a mean value of $49 \%$; no genotype showed higher value compared to the high responsive parent Express 617 (88 \%) and a lower value compared to the low responsive parent DH4079 (35\%); the mean value of the F1 (35\%) for direct embryo to plant conversion did not differ from the parental genotype DH4079. For the trait root regeneration, results of the $\mathrm{DH}$ population ranged from $14 \%$ to $95 \%$ and showed a mean value of $66 \%$. The mean value for root regeneration of the responsive parent Express 617 (96\%) significantly differed from the mean values of the low responsive parent DH4079 (42.9\%) and the F1 (52.1\%). In the studied population, the trait secondary embryogenesis varied from $4 \%$ to $91 \%$ with a mean of $38 \%$. Parental genotypes and the F1 displayed similar results. Phenotypic values of each DH line for all traits are shown in Appendix A.1. 

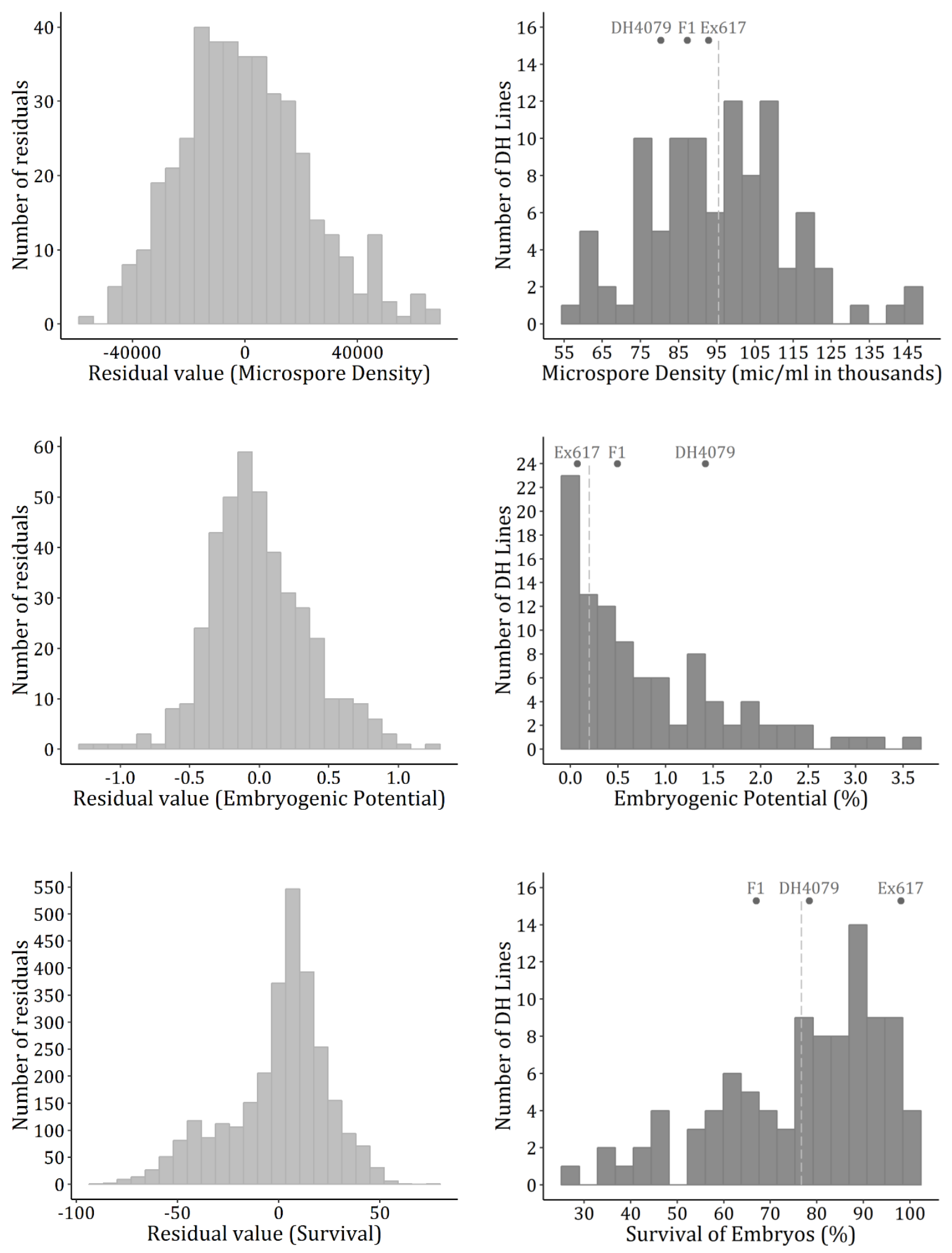

Figure 3.3 Frequency distribution of the residuals (left) and the traits (right) microspore density, embryogenic potential and survival of embryos of the DH population. Residual values are normally distributed. Frequency distribution of residuals of embryogenic potential was generated based on square root transformed data. Mean values of the DH population (vertical dashed line), of the cross (F1) and parental genotypes Express 617 (Ex617) and DH4079 (DH4079) are also presented. 

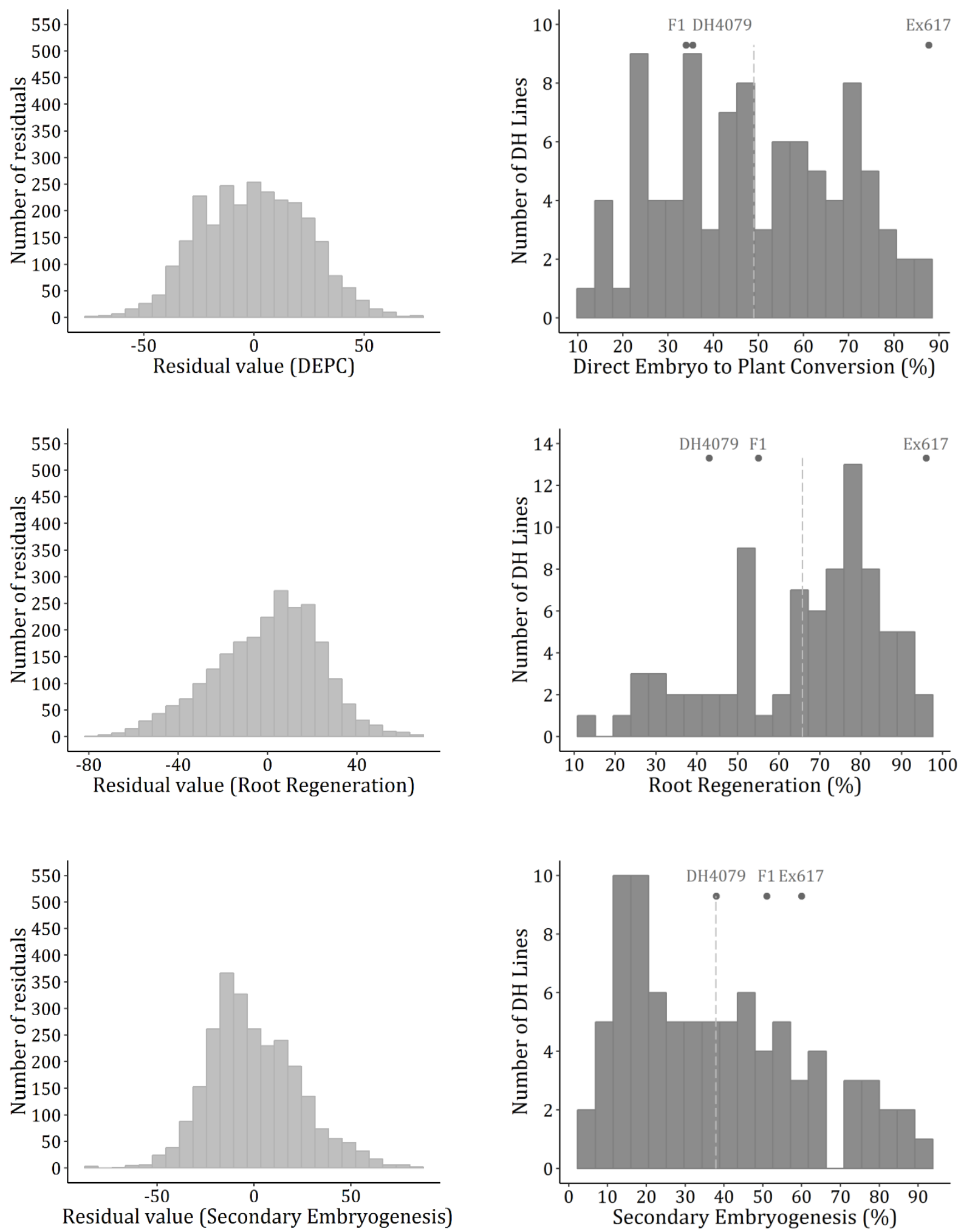

Figure 3.4 Frequency distribution of the residuals (left) and of the traits (right) direct embryo to plant conversion, root regeneration, and secondary embryogenesis of the DH population. Residual values are normally distributed. Mean values of the DH population (vertical dashed line), of the cross (F1) and parental genotypes Express 617 (Ex617) and DH4079 (DH4079) are also presented. 
Table 3.2 Descriptive statistics of the studied variables in the DH population, parental lines and progeny.

\begin{tabular}{|c|c|c|c|c|c|c|c|c|}
\hline \multirow{2}{*}{ Trait } & \multirow{2}{*}{ Min } & \multirow{2}{*}{ Max } & \multirow{2}{*}{ Mean } & \multirow{2}{*}{$\begin{array}{l}\text { LSD } \\
\mathbf{5} \%\end{array}$} & \multirow{2}{*}{ SD } & \multicolumn{2}{|c|}{ Parents ${ }^{c}$} & \multirow{2}{*}{$\mathbf{F 1}^{d}$} \\
\hline & & & & & & DH4079 & Express 617 & \\
\hline Microspore Density $(\mathrm{Mic} / \mathrm{ml})^{a}$ & 58.9 & 148.7 & 95.5 & 305.7 & 18.7 & 82.3 & 90.8 & 84.3 \\
\hline Microspore Embryogenic Potential (\%) & 0.0 & 3.6 & 0.2 & 0.24 & 0.8 & 1.4 & 0.003 & 0.5 \\
\hline Embryo Survival (\%) & 26.2 & 99.6 & 76.7 & 27.1 & 17.6 & 78.4 & 98.0 & 73.2 \\
\hline $\mathrm{DEPC}^{b}(\%)$ & 13.0 & 85.0 & 49.0 & 23.6 & 19.5 & 34.9 & 87.7 & 34.7 \\
\hline Root Regeneration (\%) & 13.6 & 95.4 & 65.8 & 22.4 & 19.6 & 42.9 & 95.6 & 52.1 \\
\hline Secondary Embryogenesis (\%) & 4.4 & 91.3 & 37.9 & 25.2 & 22.6 & 43.9 & 52.6 & 50.9 \\
\hline
\end{tabular}

a Mic $=$ number on microspores in thousands

b DEPC = direct embryo to plant conversion

c Depicted values are means over 15 experiments

$\mathrm{d}$ F1 $=$ cross DH4079 $\mathrm{x}$ Express 617 


\subsubsection{Correlation among Traits}

A correlation analysis between the means over all experiments for all traits, including direct embryo to plant conversion and the number of microspores per DH line, was performed. A weak negative correlation was found between microspore embryogenic potential and the density of microspores. A weak positive correlation was observed between the total number of microspore derived embryos and their survival after being transferred to B5 Medium. Survival of microspore derived embryos was strongly correlated with all the remaining traits: direct embryo to plant conversion, root regeneration and secondary embryogenesis. A very strong correlation was detected between the trait direct embryo to plant conversion and root regeneration. Correlation between secondary embryogenesis and direct embryo to plant conversion and root regeneration was weakly positive (Table 3.3). No correlation was identified for the readiness of direct embryo to plant conversion of the individual DH microspore derived embryos of the segregating population and the trait direct embryo to plant conversion $\left(r_{S}=-0.06\right)$.

Table 3.3 Spearman's rank correlations $\left(\mathrm{r}_{\mathrm{s}}\right)$ between microspore culture traits.

\begin{tabular}{|c|c|c|c|c|c|c|}
\hline Trait & $\operatorname{MIC} a$ & POT $^{b}$ & SURV $c$ & $\mathrm{DEPC}^{d}$ & ROOTe & SEEM \\
\hline POT $^{b}$ & $-0.17^{*}$ & & & & & \\
\hline SURV $c$ & 0.07 & 0.13 & & & & \\
\hline DEPC $^{d}$ & 0.05 & 0.10 & $0.78^{* *}$ & & & \\
\hline ROOTe & -0.05 & 0.13 & $0.78^{* *}$ & $0.66^{* *}$ & & \\
\hline SEEM $f$ & 0.14 & 0.01 & $0.58^{* *}$ & $0.37^{* *}$ & $0.37^{* *}$ & \\
\hline READ $g$ & $0.16^{*}$ & $-0.20 * *$ & -0.04 & -0.06 & $-0.14^{*}$ & -0.06 \\
\hline \multicolumn{7}{|c|}{$*$ and ${ }^{* *}$ denotes significance at $\mathrm{P}<0.05$ and 0.01 respectively } \\
\hline \multicolumn{7}{|c|}{ a MIC = Number of microspores } \\
\hline \multicolumn{7}{|c|}{ b POT = Microspore Embryogenic Potential } \\
\hline \multicolumn{7}{|c|}{ c SURV = Survival } \\
\hline \multicolumn{7}{|c|}{ d DEPC = Direct Embryo to Plant Conversion } \\
\hline \multicolumn{7}{|c|}{ e ROOT = Root Regeneration } \\
\hline \multicolumn{7}{|c|}{ f SEEM = Secondary Embryogenesis } \\
\hline \multicolumn{7}{|c|}{$\begin{array}{l}\mathrm{g} \text { READ = Readiness of the DEPC of individual DH microspore derived embryos of the segregating } \\
\text { population }\end{array}$} \\
\hline
\end{tabular}




\subsubsection{Genetic Map and Marker Segregation}

The developed molecular map consisted of 1,414 SNP markers distributed over 19 linkage groups (Table 3.4). Linkage group A07 was the largest chromosome of the DH population with $209.70 \mathrm{cM}$ length followed by C03 and C06 with 187.40 and $180.00 \mathrm{cM}$, respectively, even though A03 had the largest marker number with 114 SNPs. The average marker interval was $1.69 \mathrm{cM}$. The smallest linkage groups corresponded to A04, A08 and A10 with only 67.70, 79.10 and $76.20 \mathrm{cM}$ length. Large gaps without segregating markers were detected on A07 (40.7 cM) and C06 (37.3 cM).

A significant deviation of the expected Mendelian segregation ratio of 1:1 was observed in the population of 204 DH lines. $48.9 \%$ of the SNP markers exhibited distorted segregation $\left(X^{2} \geq 3.84, P \leq 0.05\right)$, from which $63.4 \%$ showed a significantly higher distortion towards the alleles of the responsive cultivar DH4079, and only $36.6 \%$ towards the parental inbreed line Express 617. Loci with distorted marker segregation were not randomly distributed along the genome and such distribution was mostly consistent among the 4 subpopulations constituting the DH population. Markers exhibiting an excess of DH4079 alleles clustered within linkage groups A03, A06, A07, A08, C03, C05, C07 and C09 (Figure 3.5 and 3.6). Markers showing an excess of Express 617 were predominantly present in linkage groups A01, A02, A09, A10, C02 and C06. Linkage groups A05, C01, C04 and C08 contained markers with distorted segregation in favor of both parental genotypes, which clustered in different regions along the chromosome. Distorted segregation was not observed in linkage group A04. 
Table 3.4 Marker distribution, size and mean distances between markers of each linkage group of the A and C genomes in the linkage map of the DH population DH4079 x Express 617.

\begin{tabular}{|c|c|c|c|c|c|c|}
\hline \multirow{2}{*}{$\begin{array}{l}\text { Linkage } \\
\text { Group }\end{array}$} & \multicolumn{3}{|c|}{ No. of markers } & \multirow{2}{*}{$\begin{array}{l}\text { Length } \\
\text { [cM] }\end{array}$} & \multirow{2}{*}{$\begin{array}{c}\text { Average marker } \\
\text { interval } \\
{[\mathrm{cM}]}\end{array}$} & \multirow{2}{*}{$\begin{array}{l}\text { Biggest } \\
\text { Gap } \\
\text { [cM] }\end{array}$} \\
\hline & $\begin{array}{c}\text { Polymorphic } \\
\text { markers }\end{array}$ & $\begin{array}{c}\text { Map } \\
\text { development }\end{array}$ & $\begin{array}{c}\text { Framework } \\
\text { map markers }\end{array}$ & & & \\
\hline A01 & 1013 & 290 & 83 & 147.10 & 1.77 & 11.5 \\
\hline A02 & 992 & 196 & 84 & 103.60 & 1.23 & 3.9 \\
\hline A03 & 1264 & 171 & 114 & 121.30 & 1.06 & 3.5 \\
\hline A04 & 777 & 130 & 60 & 67.70 & 1.13 & 12.1 \\
\hline A05 & 1089 & 171 & 72 & 116.60 & 1.62 & 12.1 \\
\hline A06 & 872 & 169 & 86 & 111.20 & 1.29 & 5.9 \\
\hline $\mathrm{A} 07$ & 1101 & 267 & 78 & 209.70 & 2.69 & 40.7 \\
\hline A08 & 912 & 109 & 52 & 79.10 & 1.52 & 5.9 \\
\hline A09 & 1079 & 140 & 79 & 135.80 & 1.72 & 18.8 \\
\hline A10 & 905 & 137 & 68 & 76.20 & 1.12 & 10.4 \\
\hline C01 & 2244 & 217 & 70 & 107.00 & 1.53 & 10.6 \\
\hline $\mathrm{C} 02$ & 2315 & 197 & 74 & 103.60 & 1.40 & 11 \\
\hline $\mathrm{C} 03$ & 1108 & 186 & 101 & 187.40 & 1.86 & 14.6 \\
\hline C04 & 2487 & 137 & 90 & 142.20 & 1.58 & 8.9 \\
\hline C05 & 519 & 117 & 64 & 121.40 & 1.90 & 5.9 \\
\hline C06 & 1138 & 191 & 64 & 180.00 & 2.81 & 37.3 \\
\hline $\mathrm{C} 07$ & 1468 & 131 & 68 & 122.40 & 1.80 & 13.9 \\
\hline C08 & 836 & 91 & 52 & 107.20 & 2.06 & 6.9 \\
\hline C09 & 595 & 96 & 55 & 106.00 & 1.93 & 14.8 \\
\hline A Genome & 10004 & 1780 & 776 & 1168.30 & 1.52 & 40.7 \\
\hline C Genome & 12710 & 1363 & 638 & 1177.20 & 1.87 & 37.3 \\
\hline Total & 22714 & 3143 & 1414 & 2345.50 & 1.69 & 40.7 \\
\hline
\end{tabular}




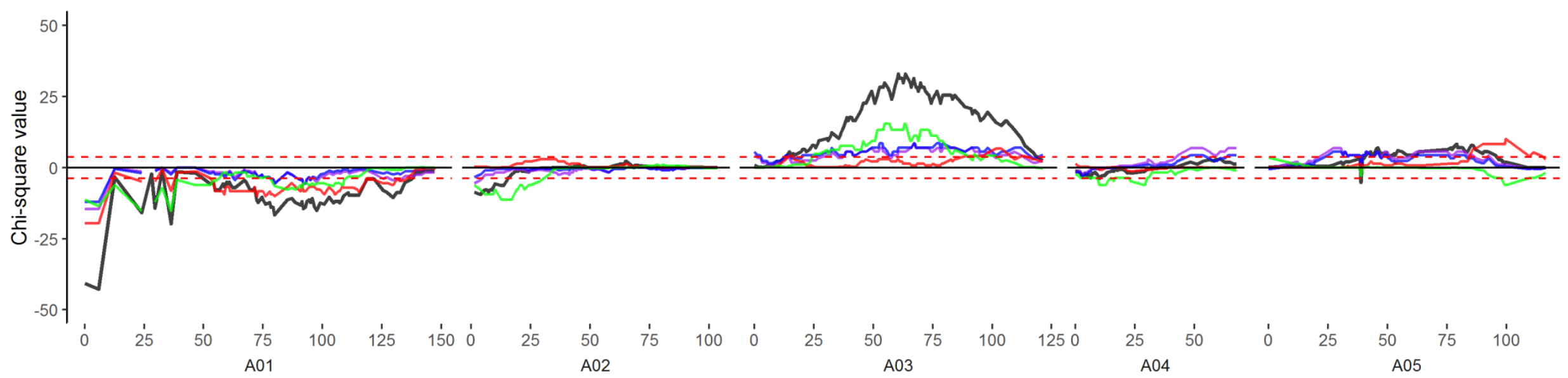

Position (cM)

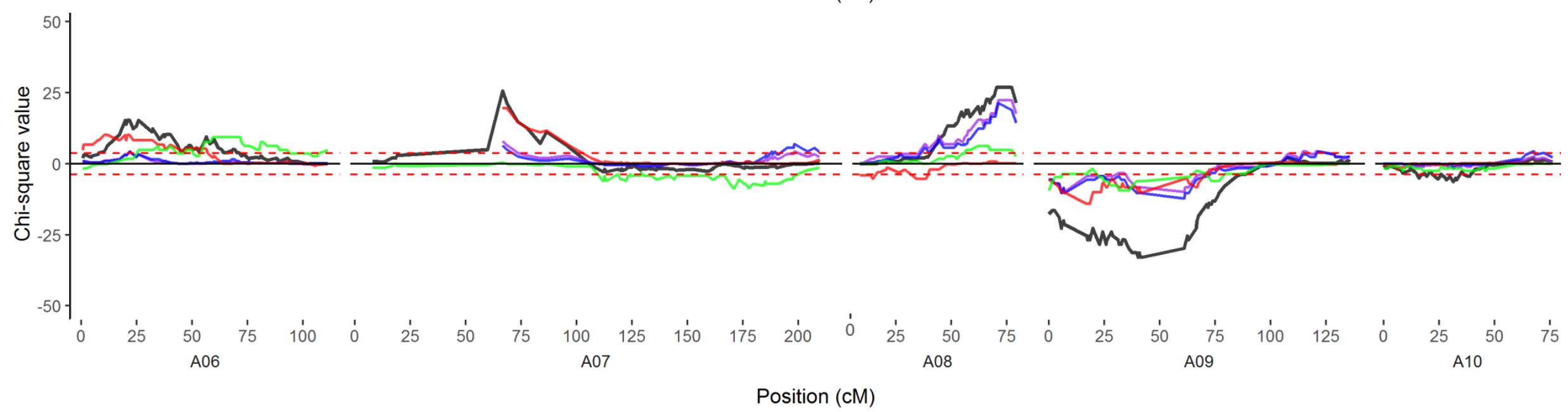

Figure 3.5 Distribution of distorted marker segregation along the 19 linkage groups in the DH population DH4079 x Express 617 (black line). Distorted marker segregation of the 4 subpopulations is defined by colored lines. Significant skewed marker segregation $(\mathrm{P}<0.05)$ above the absolute Chi-squared value $\left(\mathrm{x}^{2}=3.84\right)$ is represented by a dashed red line $(---)$. Alleles segregating in favor of parental genotype DH4079 have positive values, alleles segregating in favor of parental genotype Express 617 have negative values. 


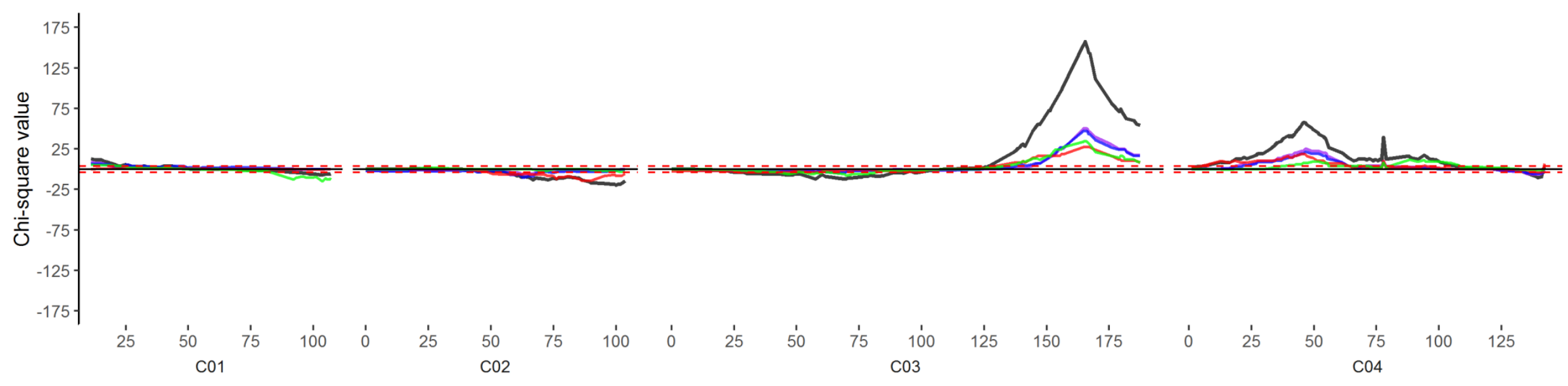

Position (CM)

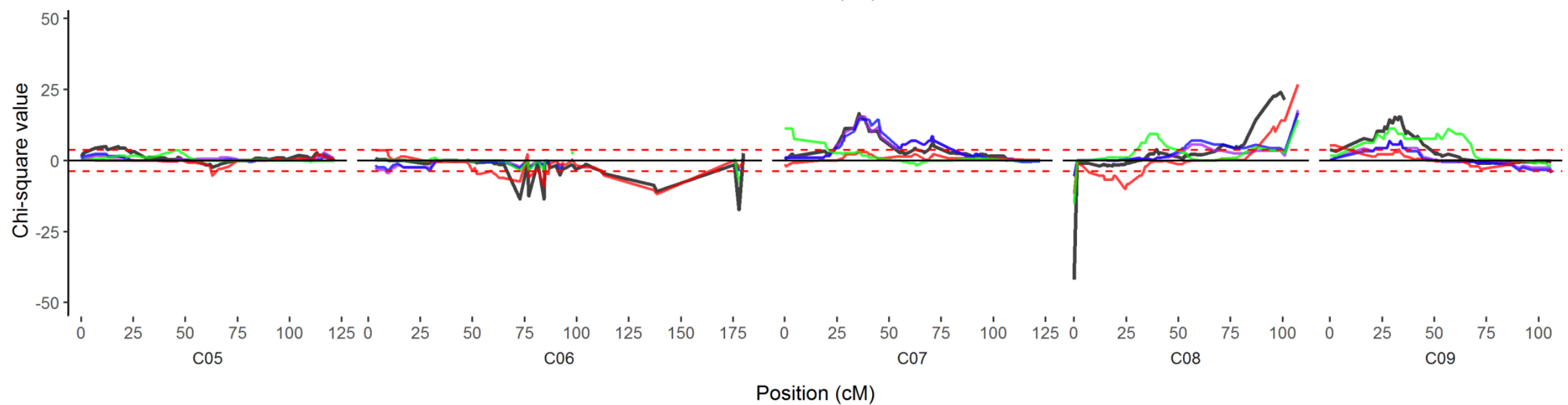

Figure 3.5 (Continue from previous page) Distribution of distorted marker segregation areas along the 19 linkage groups in the DH population DH4079 x Express 617 (black line). Distorted marker segregation of the 4 subpopulations is defined by colored lines. Significant skewed marker segregation $(\mathrm{P}<0.05)$ above the absolute Chi-squared value $\left(\mathrm{x}^{2}=3.84\right)$ is represented by a dashed red line $\left(--{ }^{-}\right)$. Alleles segregating in favor of parental genotype DH4079 have positive values, alleles segregating in favor of parental genotype Express 617 have negative values. 


\subsubsection{QTL analysis}

QTL analysis allowed the identification of ten QTL for the different traits (Table 3.5 and Figures 3.6 and 3.7). Eight QTL were identified in the A genome, while only two QTL were positioned in C genome. No QTL was detected for the trait density of microspores.

Three QTL were found for the trait microspore embryogenic potential, which together explained $31.7 \%$ of the phenotypic variance observed in the DH population. The QTL POT.A01.67 and P0T.C06.134 were located in linkage groups A01 and C06, respectively, positioned in areas of distorted marker segregation in favor of Express 617. The QTL POT.A02.73 was located in chromosome A02 in a region without biased segregation. The three QTL identified for the trait microspore embryogenic potential displayed negative additive effects. This indicates that the alleles increasing the trait value are derived from the parental genotype Express 617.

Only one major QTL $\left(R^{2} \geq 25 \%\right)$, was determined for the trait survival of microspore derived embryos that explained $26.1 \%$ of the phenotypic variance. This QTL, SURV.A05.43, was located in a region with distorted marker segregated in favor of DH4079 on linkage group A05 and showed a negative additive affect. The QTL allele was derived from the parental genotype Express 617 which displayed a higher survival performance compared to DH4079.

Two QTL located in areas of non-distorted marker segregation on linkage groups A05 and A10 were detected for the trait secondary embryogenesis, which explained $34.8 \%$ of the phenotypic variance in the DH population. The two QTL related to secondary embryogenesis displayed additive effects in opposite directions: SECEMB.A05.21 showed a negative phenotypic effect and SECEMB.A10.20 a positive additive effect.

Two QTL explaining $35 \%$ of the phenotypic variance were identified for the trait direct embryo to plant conversion. The additive effect was negative for the QTL DEPC.A02.49, positioned in an area with non-distorted marker segregation on linkage group A02. The additive effect for QTL DEPC.A05.41 was also negative; it was located on A05 in a region with biased marker segregation in favor of DH4079. 
For the trait root regeneration two QTL, R00T.A05.41 and RO0T.C04.140, were determined in chromosome A05 and C04, which showed negative additive effects and together explained $34.5 \%$ of the phenotypic variance in the DH population. The QTL ROOT.A05.41 was located in an area with distorted marker segregation in favor of DH4079, whereas the QTL ROOT.C04.140 was located in a region with biased marker segregated in favor of Express 617.

QTL for the traits survival, secondary embryogenesis, direct embryo to plant conversion and root regeneration were located in linkage group A05. All of those QTL, except the one for secondary embryogenesis, showed small overlapping confidence intervals (Figure 3.7). 
Table 3.5 Mapped QTL and their most likely positions for the studied traits in the DH population DH4079 x Express 617.

\begin{tabular}{|c|c|c|c|c|c|c|c|c|}
\hline QTL & Trait & $\begin{array}{c}\text { Linkage } \\
\text { Group } \\
\end{array}$ & $\begin{array}{c}\text { Position } \\
\text { (cM) }\end{array}$ & $\begin{array}{c}\mathrm{CI}^{a} \\
(\mathrm{cM}) \\
\end{array}$ & LOD & $\begin{array}{c}\text { Additive } \\
\text { effect }^{b}\end{array}$ & $\begin{array}{l}\mathrm{R}^{2 c} \\
(\%) \\
\end{array}$ & $\begin{array}{l}\mathrm{TR}^{2 d} \\
(\%) \\
\end{array}$ \\
\hline POT.A01.67 & \multirow{3}{*}{ Microspore Embryogenic Potential } & $\mathrm{A} 01$ & 67 & $67-70$ & 3.35 & -0.28 & 9.60 & \multirow{3}{*}{31.7} \\
\hline POT.A02.73 & & A02 & 73 & $69-75$ & 3.23 & -0.26 & 8.07 & \\
\hline POT.C06.134 & & $\mathrm{C} 06$ & 134 & $112-144$ & 5.14 & -0.34 & 13.99 & \\
\hline SURV.A05.43 & Survival & A05 & 43 & $43-45$ & 9.39 & -0.93 & 26.09 & 26.1 \\
\hline SECEMB.A05.21 & \multirow{2}{*}{ Secondary Embryogenesis } & A05 & 21 & $2-25$ & 4.26 & -0.77 & 11.23 & \multirow[t]{2}{*}{32.5} \\
\hline SECEMB.A10.20 & & A10 & 20 & $16-23$ & 7.92 & 0.11 & 21.30 & \\
\hline DEPC.A02.49 & \multirow{2}{*}{ Direct Embryo to Plant Conversion } & A02 & 49 & $49-53$ & 3.71 & -0.07 & 12.10 & \multirow{2}{*}{35.0} \\
\hline DEPC.A05.41 & & A05 & 41 & $40-43$ & 6.49 & -0.09 & 22.90 & \\
\hline R00T.A05.41 & \multirow{2}{*}{ Root Regeneration } & A05 & 41 & $39-43$ & 3.72 & -0.07 & 11.99 & \multirow{2}{*}{34.5} \\
\hline ROOT.C04.140 & & $\mathrm{C} 04$ & 140 & $127-140$ & 6.73 & -0.09 & 22.49 & \\
\hline
\end{tabular}

a $\mathrm{CI}=$ Confidence Interval

b Negative additive effects indicate that alleles increasing the trait value originate from Express 617, while positive effects indicate the alleles increasing the trait value originate from DH4079

${ }^{c} R^{2}$ represents the percentage of phenotypic variance explained by the QTL

$\mathrm{d} \mathrm{TR}^{2}$ represents the total percentage of phenotypic variance explained by all QTL 
A01

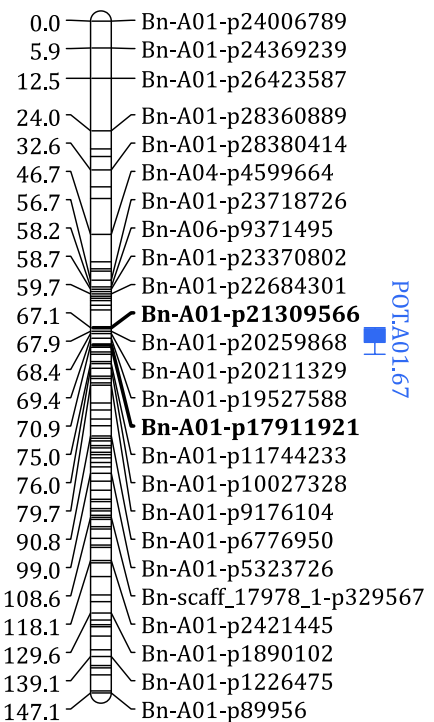

$\mathbf{A 1 0}$

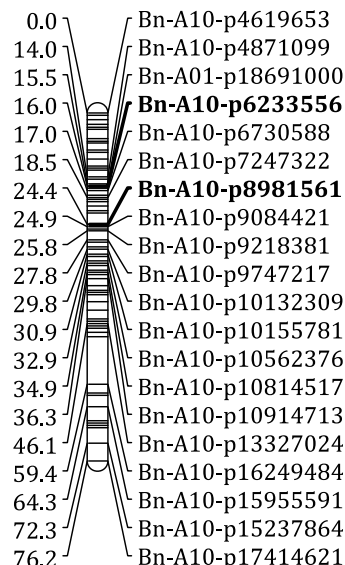

A02

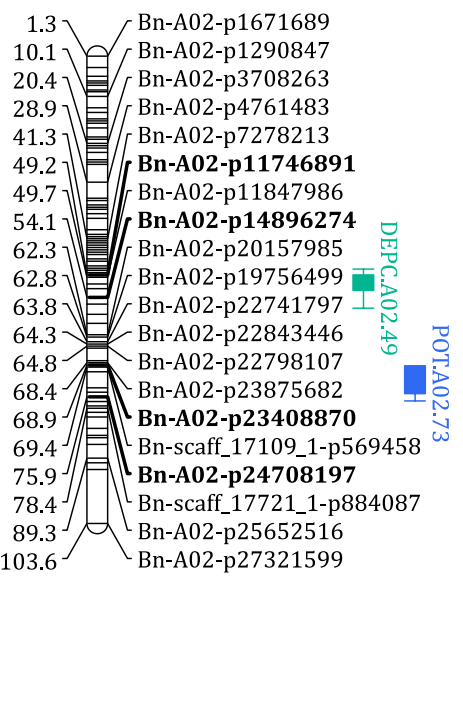

C04

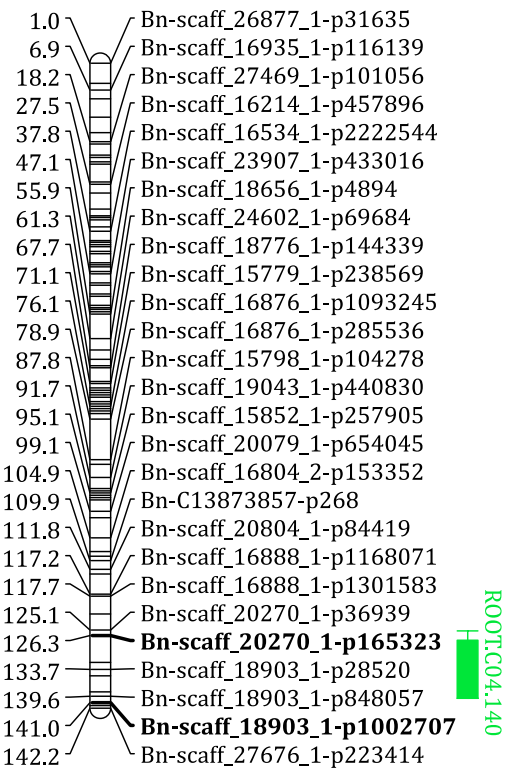

A05

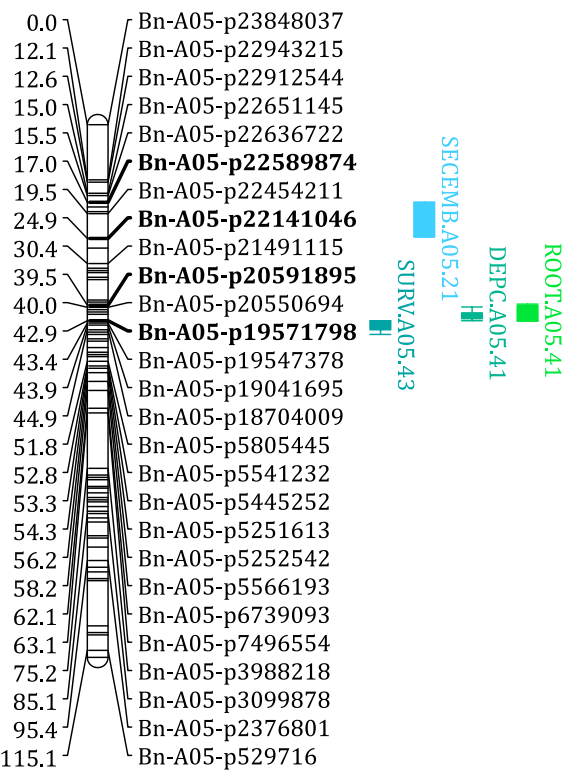

C06

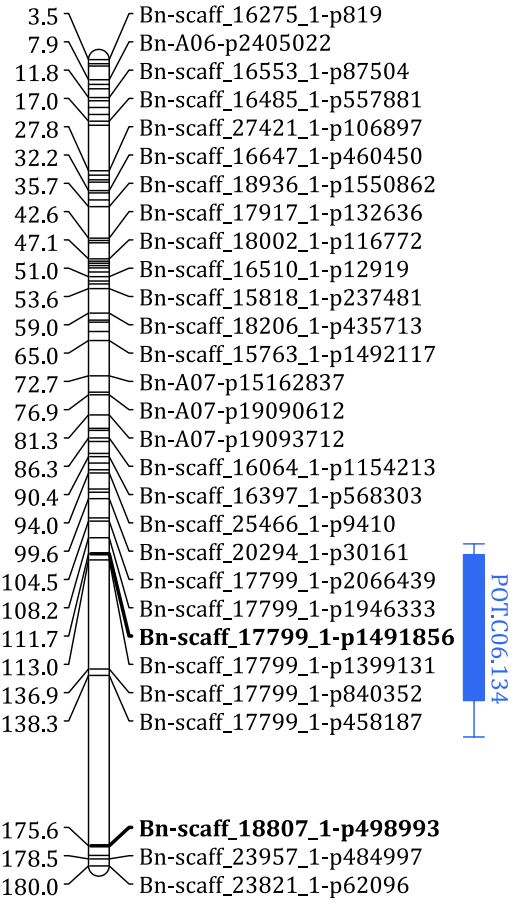

Figure 3.6 Positions of QTL for the traits microspore embryogenic potential (POT), survival (SURV), direct embryo to plant conversion (DEPC) and root regeneration (ROOT). QTL for the traits survival, direct embryo to plant conversion and root regeneration showed overlapping confidence intervals in linkage group A05. Genetic positions of markers flanking genomic regions related to the QTL are shown (bold). 
Figure 3.7 Molecular linkage map of the DH population DH4079 x Express 617. Marker segregation, mapped QTL and their most likely positions are shown.

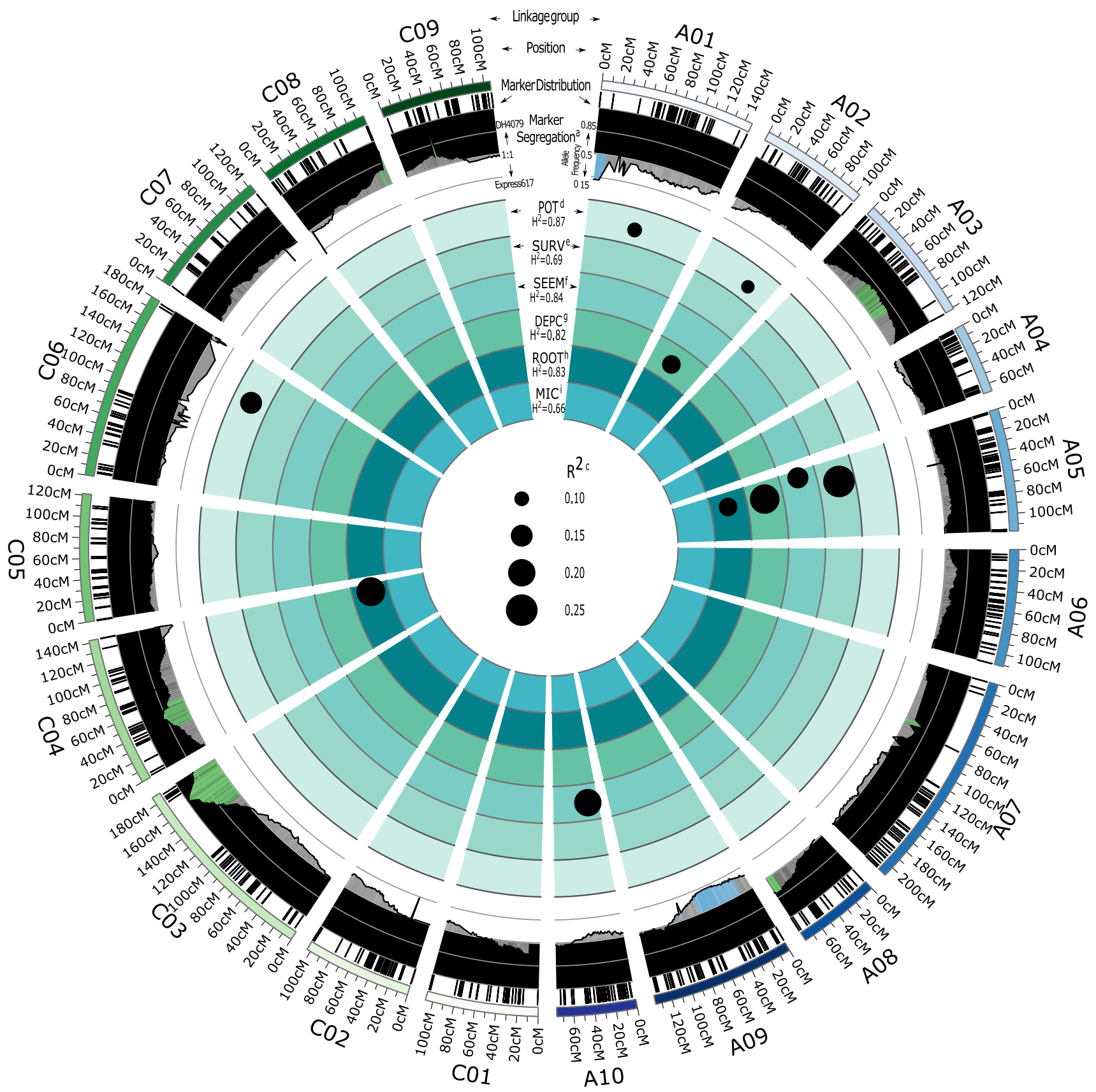

a Significant strongly distorted marker segregation $(>3: 1)$ is represented by green color when alleles segregate in favor of parental genotype D H4079, and blue color when alleles segregate towards parental genotype Express 617

${ }^{b} \mathrm{H}^{2}$ represents the value of broad-sense heritability for each trait

${ }^{c} \mathrm{R}^{2}$ represents the percentage of the phenotypic variance explained by the QTL

d POT = Embryogenic Potential

e SURV = Survival

f SEEM = Secondary Embryogenesis

$\mathrm{g}$ DEPC $=$ Direct Embryo to Plant Conversion

${ }^{\mathrm{h}} \mathrm{ROOT}=$ Root Regeneration

${ }^{\mathrm{i}} \mathrm{MIC}=$ Microspore Density 


\subsubsection{Identification of candidate genes within QTL regions}

An investigation to identify putative genes responsible for microspore culture success was performed. BLASTN analysis against the B. rapa and B. oleracea genomes indicated the presence of nineteen genes located within the $95 \%$ confidence interval of QTL related to microspore embryogenic potential, secondary embryogenesis and direct embryo to plant conversion.

Five candidate genes possibly related to microspore embryogenic potential were localized between the flanking markers of the QTL on A01 and C06 (Table 3.6 and Figure 3.8). In chromosome A01, a copy of the gene Bra028326 was found which encodes an isopentenyltransferase-7 involved in the biosynthesis of the cytokinin hormone group. The Bra037583, also located in A01, translates into a formylglycinamidine ribonucleotide synthase, associated with purine biosynthesis. Bra038210 positioned on chromosome A01, encodes a late embryogenesis abundant protein. The gene Bol042965 was located in linkage group C06. Bol042965 encodes CLE25, a homolog of the CLAVATA3 gene binding protein that is related to cell differentiation and maintenance of the meristem identity. The gene Bol016999 in C06 encodes an oleosin protein related to lipid storage.

Table 3.6 Candidate genes of B. rapa and B. oleracea located within the $95 \%$ confidence interval of the QTL accounted for microspore embryogenic potential.

\begin{tabular}{ccccl}
\hline Chromosome & Candidate Gene & $\begin{array}{c}\text { Orthologous } \\
\text { Gene in } \\
\text { A. thaliana }\end{array}$ & E value & Gene/Protein Description \\
\hline A01 & Bra028326 & AT3G23630 & $2 \mathrm{E}-126$ & $\begin{array}{l}\text { IPT7; } \\
\text { isopentenyltransferase-7 }\end{array}$ \\
A01 & Bra037583 & AT1G74260 & 5E-66 & $\begin{array}{l}\text { PUR4; formylglycinamidine } \\
\text { ribonucleotide synthase }\end{array}$ \\
A01 & Bra038210 & AT3G19430 & 4.00E-163 & $\begin{array}{l}\text { LEA; late embryogenesis } \\
\text { abundant protein-related }\end{array}$ \\
C06 & Bol042965 & AT3G28455 & 2E-17 & $\begin{array}{l}\text { CLE25; homologous to the } \\
\text { CLAVATA3 gene; protein } \\
\text { binding / receptor binding }\end{array}$ \\
& Bol016999 & AT5G61610 & $5 \mathrm{E}-43$ & Oleosin family protein
\end{tabular}

Gene descriptions were obtained from BRAD and TAIR. 


\section{Genetic map (cM) B. napus}

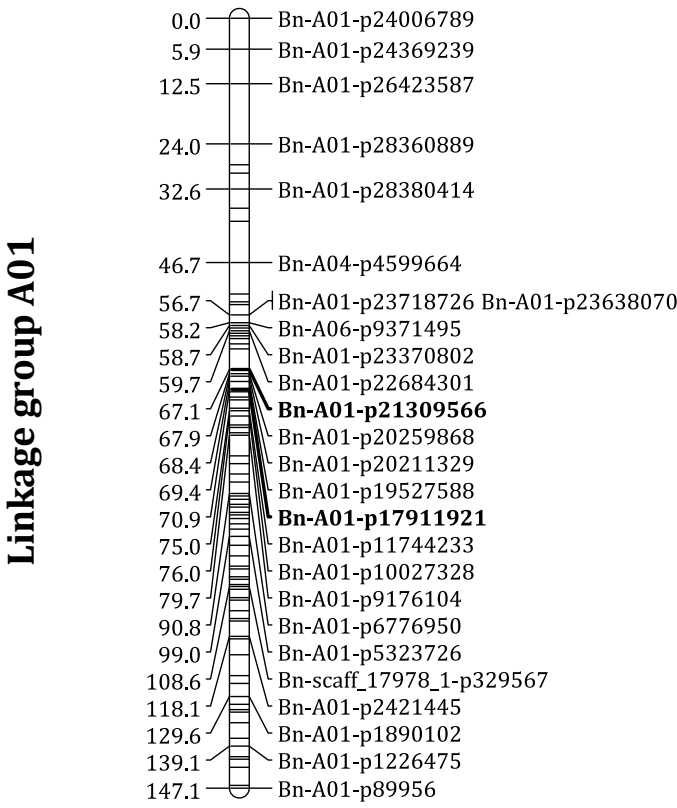

Genetic map (cM) B. napus

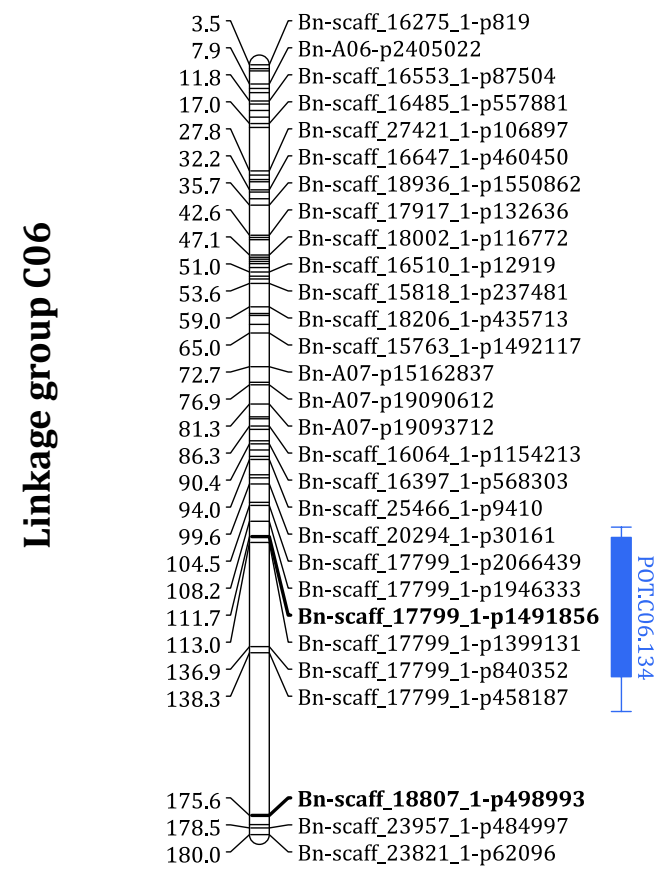

\section{Physical Map (bp) B. rapa}

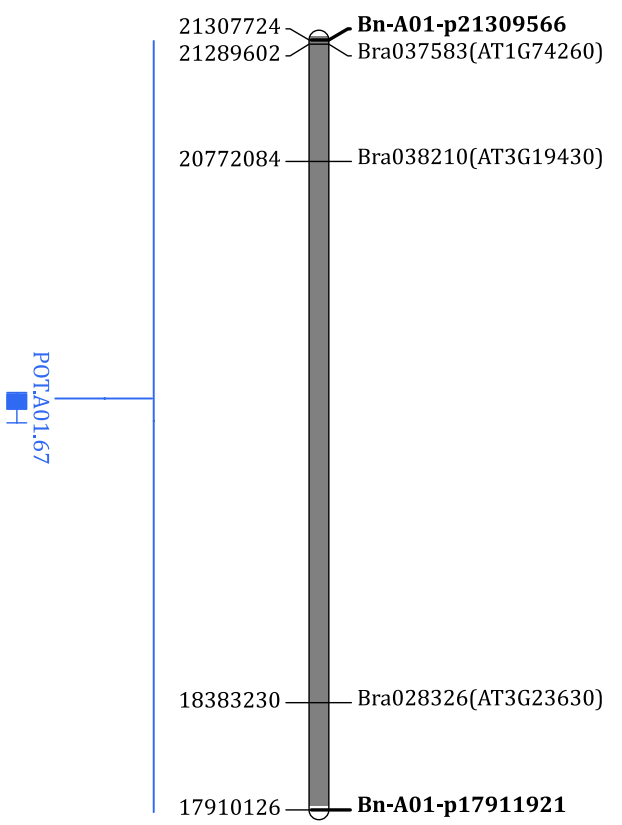

\section{Physical Map (bp) B. oleracea}

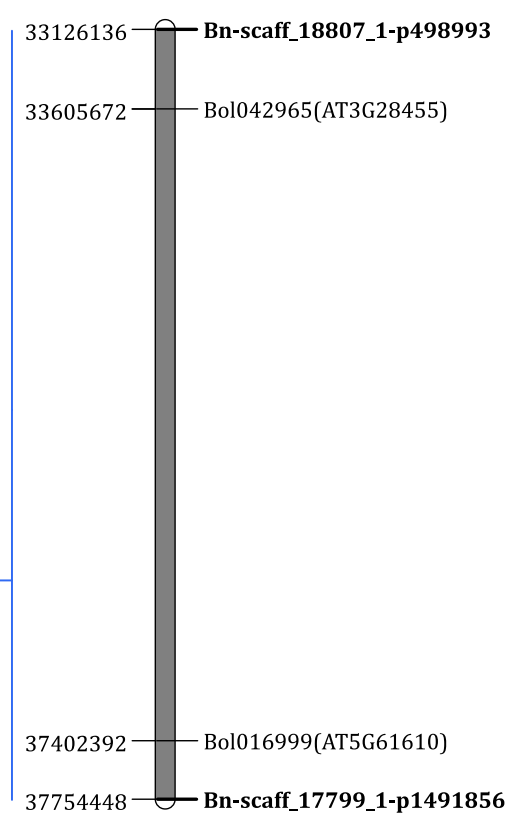

Figure 3.8 QTL positioned in linkage groups A01 and C06 associated with the trait microspore embryogenic potential (POT.A01.67 and POT.C06.7134) in the genetic map of B. napus. Physical positions of possible candidate genes within the SNP markers (bold) delimiting the $95 \%$ confidence interval of the QTL for microspore embryogenic potential in the map of B. rapa and $B$. oleracea are shown. 
Seven genes were found between the flanking markers of the QTL for secondary embryogenesis in linkage groups A05 and A10 (Table 3.7 and Figure 3.9). Five genes encode transcription factors from which Bra034855 and Bra002576, localized on chromosomes A05 and A10 respectively, encode WUSCHEL related homebox-5 and WUSCHEL related homebox-2, that are important to determine cell fates during plant development. A similar function is associated with SCARECROW transcription factor family proteins that are encoded by two genes, Bra002894 and Bra002564, located on chromosome A10 and which are responsible for maintaining stem cells in the meristems. Bra002859 on A10 is the gene of the No Apical Meristem domain transcriptional regulator superfamily protein related to the formation of boundary cells of the meristems. A gene positioned on linkage group A10, Bra002957, encodes the enzyme thiamine-4, responsible for thiamine biosynthesis and which is involved in the response to oxidative stress. Finally, a gene with unknown activity, Bra002994 localized on chromosome A10, encodes a late embryogenesis abundant related-protein.

Table 3.7 Candidate genes of B. rapa and located within the $95 \%$ confidence interval of the QTL accounted for secondary embryogenesis.

\begin{tabular}{|c|c|c|c|c|}
\hline Chromosome & Candidate Gene & $\begin{array}{c}\text { Orthologous } \\
\text { Gene in } \\
\text { A. thaliana } \\
\end{array}$ & E value & Gene/Protein Description \\
\hline A05 & Bra034855 & AT3G11260 & $1 \mathrm{E}-36$ & $\begin{array}{l}\text { WOX5; WUSCHEL related } \\
\text { homebox-5 }\end{array}$ \\
\hline A10 & Bra002576 & AT5G59340 & $3 \mathrm{E}-62$ & $\begin{array}{l}\text { WOX2; WUSCHEL related } \\
\text { homebox-2 }\end{array}$ \\
\hline A10 & Bra002894 & AT4G08250 & 0.0 & $\begin{array}{l}\text { SCARECROW transcription } \\
\text { factor family protein }\end{array}$ \\
\hline A10 & Bra002564 & AT5G59450 & $2.00 \mathrm{E}-168$ & $\begin{array}{l}\text { SCL11; SCARECROW-like } \\
\text { transcription factor-11 }\end{array}$ \\
\hline A10 & Bra002859 & AT1G60340 & $1.00 \mathrm{E}-24$ & 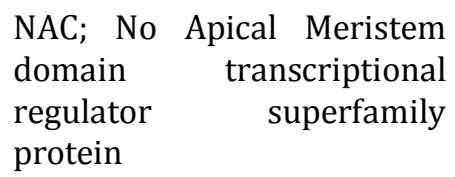 \\
\hline A10 & Bra002957 & AT5G54770 & $2 \mathrm{E}-175$ & THI4; thiamine-4 \\
\hline A10 & Bra002994 & AT5G54370 & $1.00 \mathrm{E}-138$ & $\begin{array}{l}\text { LEA; late embryogenesis } \\
\text { abundant protein-related }\end{array}$ \\
\hline
\end{tabular}

Gene descriptions were obtained from BRAD and TAIR. 


\section{Genetic map (cM) B. napus}
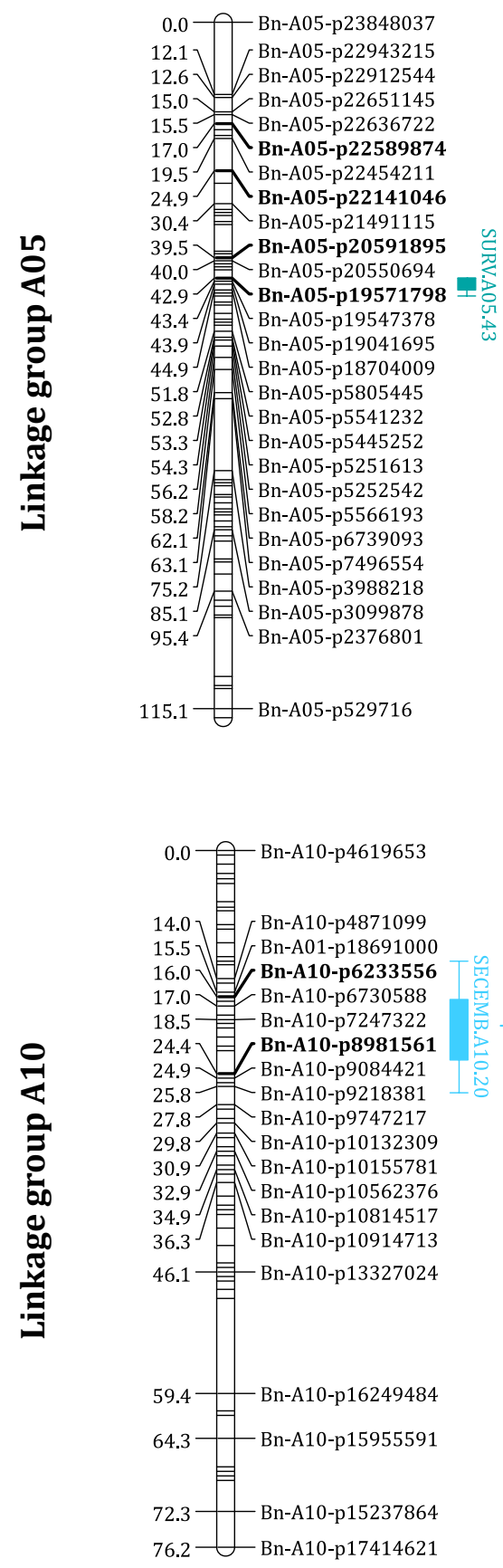

\section{Physical Map (bp) B. rapa}

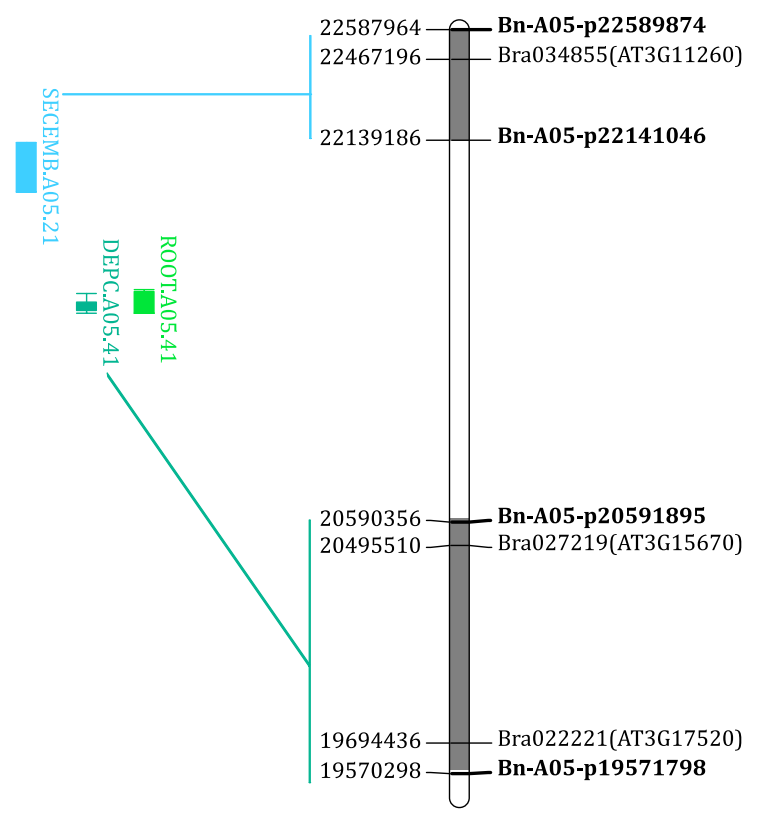

Figure 3.9 QTL positioned in linkage groups A05 and A10 associated with the traits survival (SURV.A05.43), direct embryo to plant conversion (DEPC.A05.41), root (ROOT.A05.41) regeneration and secondary embryogenesis (SEEM.A05.21, SEEM.A10.20) in the genetic map of B. napus. Physical positions of possible candidate genes within the SNP markers (bold) delimiting the $95 \%$ confidence interval of the QTL for direct embryo to plant conversion and secondary embryogenesis in the map of $B$. rapa are shown. 
Five genes possibly related to direct embryo to plant conversion were localized within the $95 \%$ confidence interval of the QTL on A02 and A05 (Table 3.8 and Figure 3.9 and 3.10). One transcription factor gene, Bra008259 on linkage group A02, encode CUPSHAPED COTYLEDON-3 which is involved in cell fate. Two enzyme genes, Bra008362 and Bra008421 on A02, encode the enzymes gibberellin 2-oxidase-1 and a phosphoglycerate kinase. Gibberellin 2-oxidase-1 is involved in hormone biosynthetic processes while the phosphoglycerate kinase is associated with glycolysis. A gene Bra008076, homologous to the CLAVATA3 gene, is positioned on chromosome A02 and encodes CLE1, a DNA-binding protein highly related to cell differentiation and maintenance of the meristem identity. Two genes, Bra022221 and Bra027219, with unknown activity were also located in the confidence intervals of the QTL for the trait direct embryo to plant conversion on linkage group A05, which encodes for late embryogenesis abundant domain-containing proteins.

Table 3.8 Candidate genes of B. rapa and located within the $95 \%$ confidence interval of the QTL accounted for direct embryo to plant conversion.

\begin{tabular}{|c|c|c|c|c|}
\hline Chromosome & Candidate Gene & $\begin{array}{l}\text { Orthologous } \\
\text { Gene in } \\
\text { A. thaliana }\end{array}$ & E value & Gene/Protein Description \\
\hline A02 & Bra008259 & AT1G76420 & $9 \mathrm{E}-127$ & $\begin{array}{l}\text { CUC3; cup shaped cotyledon- } \\
3\end{array}$ \\
\hline A02 & Bra008362 & AT1G78440 & $3.00 \mathrm{E}-143$ & $\begin{array}{l}\text { ATGA20X1; gibberellin 2- } \\
\text { oxidase-1 }\end{array}$ \\
\hline A02 & Bra008421 & AT1G79550 & 0.0 & $\begin{array}{l}\text { PGK; phosphoglycerate } \\
\text { kinase }\end{array}$ \\
\hline A02 & Bra008076 & AT1G73165 & $7 \mathrm{E}-23$ & $\begin{array}{l}\text { CLE1; homologous to the } \\
\text { CLAVATA3 gene; protein } \\
\text { binding / receptor binding }\end{array}$ \\
\hline A05 & Bra022221 & АT3G17520 & $2.00 \mathrm{E}-74$ & $\begin{array}{l}\text { LEA; late embryogenesis } \\
\text { abundant domain-containing } \\
\text { protein }\end{array}$ \\
\hline A05 & Bra027219 & АТ3G15670 & $2.00 \mathrm{E}-59$ & $\begin{array}{l}\text { LEA; late embryogenesis } \\
\text { abundant domain-containing } \\
\text { protein putative }\end{array}$ \\
\hline
\end{tabular}

Gene descriptions were obtained from BRAD and TAIR. 
Genetic map (cM) B. napus

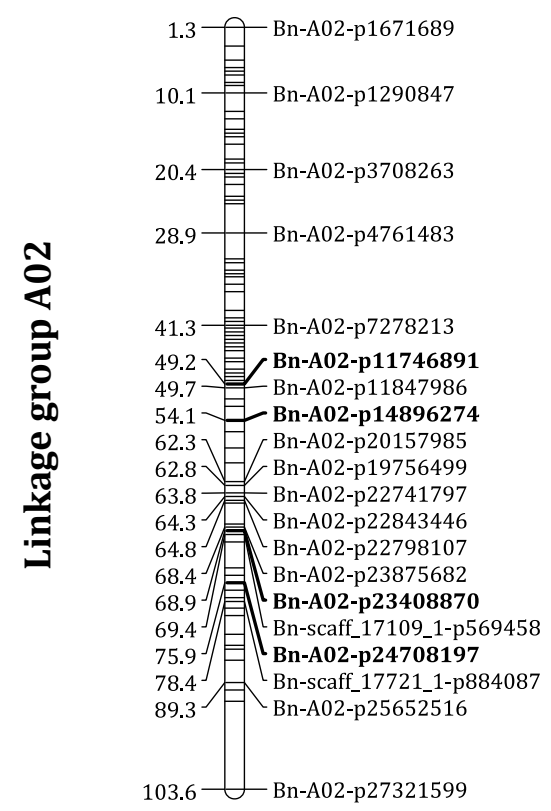

\section{Physical Map (bp) B. rapa}

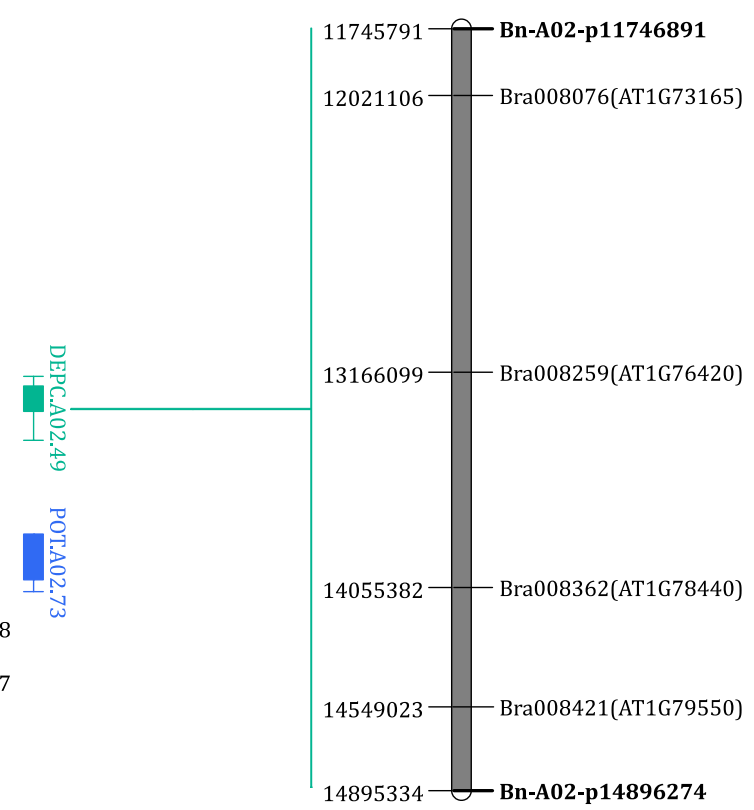

Figure 3.10 QTL positioned in linkage group A02 associated with the traits, microspore embryogenic potential (POT.A02.73) and direct embryo to plant conversion (DEPC.A02.49) in the genetic map of B. napus. Physical positions of possible candidate genes within the SNP markers (bold) delimiting the $95 \%$ confidence interval of the QTL for direct embryo to plant conversion in the map of B. rapa are shown. 


\subsection{Discussion}

Along the intensive research developed during the last decades, it has become clear that there are 3 major bottlenecks that hamper microspore culture success: diploidization, microspore embryogenic potential and direct embryo to plant conversion. In this research, the latter two traits were assessed. Secondary embryogenesis was also considered, since very similar factors may control the correct development of embryos in both the microspore and the somatic embryogenesis system. For the trait direct embryo to plant conversion, other related traits were studied: survival of embryos and root regeneration. The significance of survival of embryos is determined by the fact that the actual origin of the unsuccessful regeneration of DH plants may arise from the early death of embryos when transferring them to a suitable medium for conversion to plantlets. Therefore, survival of embryos might be considered an additional selective step during the development of double haploid populations. Independent scoring of root regeneration was performed with the aim to evaluate the presence of DH lines with the ability to form only roots but no shoots.

\subsubsection{Phenotypic variation in the DH4079 $x$ Express 617 population}

In Brassica napus, very early studies showed the potential of microspores to produce embryos (Thomas and Wenzel 1975 cited in Lichter 1982). It was observed that different hybrids with a common parent had similar behavior under the same culture conditions, revealing the genetic control of androgenesis (Dunwell and Cornish 1985). In concordance to this, a variety of oilseed rape lines have exhibited enormous differences in microspore embryogenic potential (Chuong et al. 1988; Dunwell et al. 1983; Siebel and Pauls 1989; Thurling and Chay 1984). In this study, a huge variation in response was found in the DH4079 x Express 617 population, which made clear the presence of a complex genetic network involved in microspore embryogenic potential and direct embryo to plant conversion. The significance of genetic factors involved in the in vitro culture related traits was supported by the relatively high broad-sense heritability values observed in the DH4079 x Express 617 DH population (see Table 3.1). A heritability value of $86 \%$ was identified for the trait microspore embryogenic potential. In agreement with that, Zhang and Takahata (2001) found very high broad- 
sense heritabilities of microspore embryogenic ability of $97 \%$ and $96 \%$ for B. napus and B. campestris ssp. pekinensis, respectively. Seo et al. (2013) reported heritabilities of in vitro shoot regeneration from hypocotyls in B. rapa of $75 \%$, while in the DH4079 x Express 617 DH population a heritability value of $82 \%$ was observed for direct embryo to plant conversion.

Since the embryogenic potential of microspores in Brassicas has been evaluated under very different culture conditions, it is very difficult to compare results among scientific studies. Nonetheless, all of them concluded that there is a small percentage of young pollen grains which switch their developmental pathway from gametophytic to sporophytic (Chuong and Beversdorf 1985; Dunwell and Cornish 1985; Gland et al. 1988; Iqbal et al. 1994). Results obtained in this research showed that microspore embryogenic potential ranged from 0 to $3.9 \%$, meaning that up to 43,000 embryos per 16 buds as a mean of five experiments could be obtained for very successful DH lines. Even, when many authors claimed that the optimal microspore density to obtain the highest microspore embryogenic potential ranges between 10,000 and 100,000 microspores/ml (Huang et al. 1990; Orr et al. 1990; Telmer et al. 1992; Weber et al. 2005), the microspore density of the cultures in the DH4079 x Express $617 \mathrm{DH}$ population varied broadly from 58,900 to 148,700 microspores/ml and microspore embryogenesis was achieved for 96 of the 99 studied DH lines. However, only a weak negative linear correlation was identified among the traits microspore density and microspore embryogenic potential. Such result may be derived from the fact that the relationship between those two variables could be curvilinear. Another factor influencing the lack of strong correlation among those traits could be related to the developmental stage of microspores. Late uninucleate stage has shown to be the optimal condition to generate microspore derived embryos in the cultivar Topas (Fan et al. 1988; Pechan and Keller 1988). However, a developmental asynchrony of microspores has been detected in the same anther and buds of the same plant and between individuals in similar development stage in some genotypes (Kott et al. 1988a; Pechan and Keller 1989; Kontowski and Friedt 1994), while in others, a more homogeneous microspore population has been found increasing the embryo yield (Kontowski and Friedt 1994). In the present research, the quantity of microspores at the optimal developmental phase was not recorded and would be interesting to assess 
in order to evaluate the presence of a linear correlation between microspore density and microspore embryogenic potential.

In previous studies, hybrids derived from multiples crosses of high and low responsive genotypes showed variable responses, displaying a superior performance on embryo yield compared to the parental genotypes or a value in between both parents (Kontowski and Friedt 1994; Zhang and Takahata 2001; Zhang et al. 2003), consistent with the F1 value for microspore embryogenic potential $(0.49 \%)$ of the present research, as well as for the traits root regeneration and secondary embryogenesis. This result could be the outcome of a cross between DH4079, a highly responsive genotype (Ferrie 2003), and Express 617, very recalcitrant (Klutschewski 2013; Ecke et al. 2015).

Direct embryo to plant conversion ranged from $13 \%$ to $85 \%$ in the DH population. Surprisingly, the F1 showed a value of $34.7 \%$, which was not significantly different from the less responsive parent DH4079 (34.9\%), but much below the $87.7 \%$ of Express 617, which has been recognized before for its excellent direct embryo to plant conversion (Klutschewski 2013). Such F1 response was also observed for embryo survival, even though the trait values for the parental genotypes were not statistically different based on the LSD ( $c f$. Tables 3.1 and 3.2). Klutschewski (2013) evaluated the conversion of embryos into plants in 5 cultivars and 8 crosses of oilseed rape, identifying a very contrasting response among the hybrids compared to the parents. Thus, while some hybrids performed similar to either one of the parental genotypes, others showed lower direct embryo to plant conversion values in comparison to both parents.

A correlation between the readiness of direct embryo to plant conversion of the individual DH microspore derived embryos of the segregating F1 derived embryo population and their performance for direct embryo to plant conversion was expected since the early development of a shoot could be an evidence of a genetic predisposition to easily convert directly into plants. Nonetheless, both traits showed no correlation. Genetic factors controlling direct embryo to plant conversion may be independent of the timing of shoot development. 
16 genotypes of the DH population showed a higher microspore embryogenic potential compared to the high responsive parental line DH4079, which suggested transgressive segregation. Parental genotypes DH4079 and Express 617 present contrasting response for microspore embryogenic potential and direct embryo to plant conversion, which is usually a consequence of fixed allele sets with opposite effects. Transgressive segregation in the microspore derived DH population studied in the present research could be then explained by the recombination of positive parental alleles with additive effects of complementary genes. However, QTL analysis revealed only alleles with positive additive effects from the parent Express 617. Unmasking of recessive alleles expressed in the DH derived progeny is a possibility that should not be discarded since Express 617 is an inbred line and complete homozygosity is not guaranteed (Rieseberg et al. 1999).

\subsubsection{Distorted segregation in the DH4079 x Express 617 DH population}

Skewed marker segregation has been reported very often in microspore derived DH populations of Brassica species (for a review see Ferrie and Möllers 2011). Biased marker segregations ranged from $9 \%$ to $65 \%$ and it occurred mostly in favor of the responsive parental genotype. Skewed marker segregation is considered to be caused by linkage of those markers to a gene or genes involved in microspore embryogenesis, diploidization, survival of embryos and consequently plant regeneration (Gupta 2013). As a result, specific parental alleles favoring androgenesis and/or direct embryo to plant conversion would occur more frequent in a DH population generated by microspore culture. In order to confirm the specific effect of loci on microspore culture responsiveness, skewed marker segregation should not occur in F2 populations of the same cross (Lombard and Delourme 2001). However, distorted segregation has been also reported in F2 (Chyi et al. 1992; Teutonico and Osborn, 1994), BC- (Ecke et al., 2015) and RIL- Brassica populations (Ding et al. 2012; Yu et al. 2013), which implies that factors influencing skewed allele frequency are already effective during meiosis and are not necessarily restricted to embryogenic processes.

In the DH4079 x Express 617 DH population skewed marker allele segregation was identified for $48.9 \%$ of the SNP markers. SNP markers showing strongly skewed segregation in the DH population did show the expected 1:1 Mendelian segregation in 
the $\mathrm{BC}_{1}$ population, except for one out of 15 markers, Bn-A09-p23144404 in linkage group A09 (Freudenthal 2016). Such outcome confirmed that factors influencing skewed allele frequency are not effective during meiosis and could be related to the embryogenic process. $63.4 \%$ of the SNP markers were biased in favor of the highly responsive parent DH4079 and $36.6 \%$ towards the recalcitrant parental genotype Express 617 for the trait microspore embryogenic potential in the DH population. This is in accordance with results of Tanhuanpää et al. (1994), who found that 7 out of 15 RAPD markers showed distorted segregation in favor of the highly responsive Topas in a microspore derived DH population from the cross Topas x R4. Cloutier et al. (1995) reported $23 \%$ and $31 \%$ of RFLP markers with distorted segregation in two microspore derived populations, from which $59 \%$ and $60 \%$ were biased towards the responsive parental genotype Topas and $41 \%$ and $40 \%$ in favor of the poor responsive genotype Westar. In contrast to the observed outcome in the DH4079 x Express 617 population, $31 \%$ of the mapped markers in a DH population from the cross Express x R53 showed a skewed segregation ratio with $69.3 \%$ of the alleles favoring the low responding parent Express (Radoev 2007). However, the microspore embryogenic potential of R53 is not known (Radoev 2007). Ecke et al. (2015) identified biased segregation in $48 \%$ of the markers mapped in a segregating population of haploid microspore derived embryos of a cross between Express 617 and the resynthesized oilseed rape line RS239. 19 out of 34 regions with skewed marker segregation were in favor of the parental genotype RS239. When the segregation distortion was studied in a population of DH plantlets derived from the same parental lines, $59.9 \%$ of the markers showed biased segregation. $50 \%$ of such genomic regions biased towards Express 617 alleles and $50 \%$ in favor of RS239 alleles (Kampouridis et al. 2016). Microspore embryogenic potential of the genotype RS239 is not known.

Even though previous studies suggested that gametic selection during androgenesis is a major factor acting in microspore embryogenic potential (Cloutier et al. 1995; Foisset and Delourme 1996), posterior selection during the development of embryos and plantlet regeneration could take place. Radoev et al. (2008), Ecke et al. (2015) and Kampouridis et al. (2016) identified numerous genomic regions with skewed segregation towards the androgenic recalcitrant but high direct embryo to plant conversion responsive parental genotype Express 617. Based on those results, segregation pattern in $\mathrm{DH}$ populations generated by microspore culture would be 
influenced by the differences in the response of the parental genotypes with respect to their embryogenic, diploidization and direct embryo to plant conversion potential. Since DH4079 and Express 617 were chosen according to their extremely contrasting androgenic responses, it could be expected that selection during microspore embryogenesis would play a major role and skewed segregation would be mostly in favor of the parental responsive line DH4079, even though further selection during direct embryo to plant conversion, in which Express 617 displayed a higher direct embryo to plant conversion, could also influence marker allele segregation.

\subsubsection{QTL analysis}

In the present research, 10 QTL were detected: three for microspore embryogenic potential, one for survival of the embryos, and two each for secondary embryogenesis, direct embryo to plant conversion and for root regeneration. This represents the first QTL study assessing microspore embryogenic potential and direct embryo to plant conversion in Brassica species.

In the present study, QTL for microspore embryogenic potential were located on linkage groups A01, A02 and C06, and QTL of the trait secondary embryogenesis were located in chromosomes A05 and A10. Compatible with our findings, Ecke et al. (2015) indicated the presence of important regions for microspore embryogenic potential in chromosomes A01, A02, A05 and A10.

The QTL DEPC.A02.49 on chromosome A02 was responsible for $12.1 \%$ of the phenotypic variance for direct embryo to plant conversion. The second QTL DEPC.A05.41 explained $22.15 \%$ of the phenotypic variance and its presence was supported by the QTL ROOT.A05.41 that explained $12 \%$ of the phenotypic variance for the trait root regeneration. Kampouridis et al. (2016) identified two important regions on linkage groups A02 and A05 possibly influencing direct embryo to plant conversion. In a previous research, the ability of hypocotyls to generate callus and shoots in B. rapa was evaluated (Seo et al. 2013). A QTL responsible for callus regeneration explaining $9.2 \%$ of the phenotypic variance was identified in linkage group A02. It can then be assumed that chromosome A02 carries important genes influencing in vitro direct shoot regeneration ability of B. napus. Additional to the previously mentioned QTL localized in 
linkage group A05, a QTL SURV.A05.43 in the same chromosome explained $26.1 \%$ of the phenotypic variance observed in the population for the survival of embryos. Overlapping confidence intervals of QTL in linkage group A05 indicate the presence of a clustered region carrying important factors responsible for the direct embryo to plant conversion. Alternatively, one locus in this region may exert pleiotropic effects on different traits. Significant and high correlation coefficients $\left(r_{s}=0.66-0.78, P<0.01\right)$ for embryo survival, direct embryo to plant conversion and root regeneration indicates the presence of a 'hotspot' for loci controlling the regeneration of plants resulting from microspore derived embryos. An additional QTL, ROOT.C04.140, was found on chromosome C04 that explained $22.5 \%$ of the phenotypic variance. Since a significant Spearman's correlation $\left(\mathrm{r}_{\mathrm{s}}=0.66, \mathrm{P}<0.01\right)$ was found between root regeneration and direct embryo to plant conversion, it is likely that a gene or genes related to plant regeneration are located in this area of chromosome $\mathrm{C} 04$.

Seo et al. (2013) reported the presence of two QTL on linkage group A06 and of two more on linkage groups A07 and A09 for plant regeneration, explaining $12.3 \%, 14.0 \%$, $8.4 \%$ and $11.2 \%$ of the phenotypic variance in the B. rapa population, respectively. In contrast, neither in the DH4079 x Express 617 DH population nor in the Express $617 \mathrm{x}$ RS239 DH populations (Ecke et al. 2015; Kampouridis et al. 2016), evidence for the presence of QTL related to direct embryo to plant conversion on such chromosomes was identified.

Although a large number of genomic regions with distorted marker segregation in the direction of both parents were identified ( $c f$. Figures 3.5 and 2.6), no QTL influencing microspore embryogenic potential and direct embryo to plant conversion were identified on linkage groups A03, A04, A08 and C01, C02, C03, C05 and C09. The lack of collocation of distorted marker regions on this chromosomes with QTL for microspore embryogenic potential and direct embryo to plant conversion are consistent with results obtained by Ecke et al. (2015), Kampouridis et al. (2016) and Seo et al. (2013).

Physical positions of QTL detected in the DH population DH4079 x Express 617 and physical positions of putative genomic regions related to microspore embryogenic potential and direct embryo to plant conversion detected by Ecke et al. (2015) and Kampouridis et al. (2016) were compared. Even though conspicuous genomic regions 
were found in linkage groups A01, A02, A05 and A10 for all populations, there were no overlapping regions accounting for any trait (e. g. Figure 3.11). This supports the hypothesis about the existence of many different loci influencing embryogenesis of $B$. napus in those linkage groups.

\section{Genetic map (cM) B. napus}

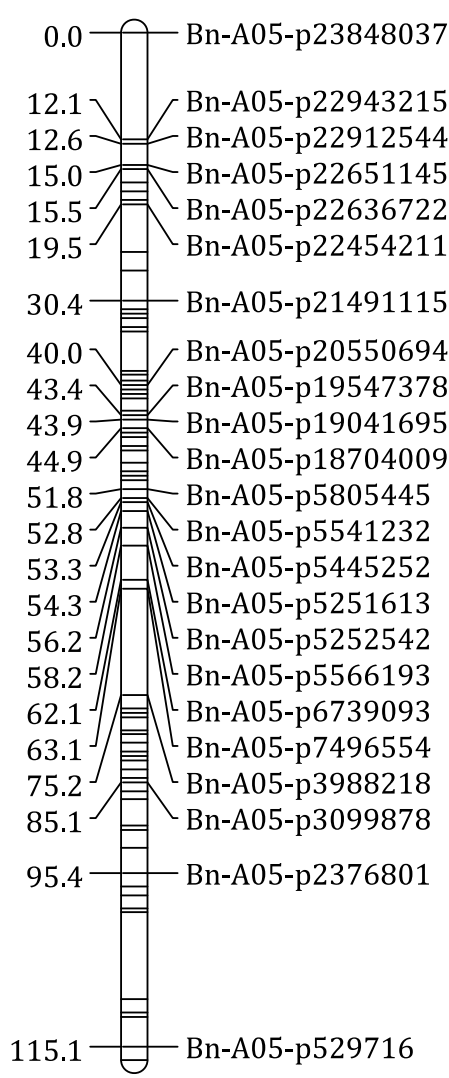

\section{Physical Map (bp) B. rapa}

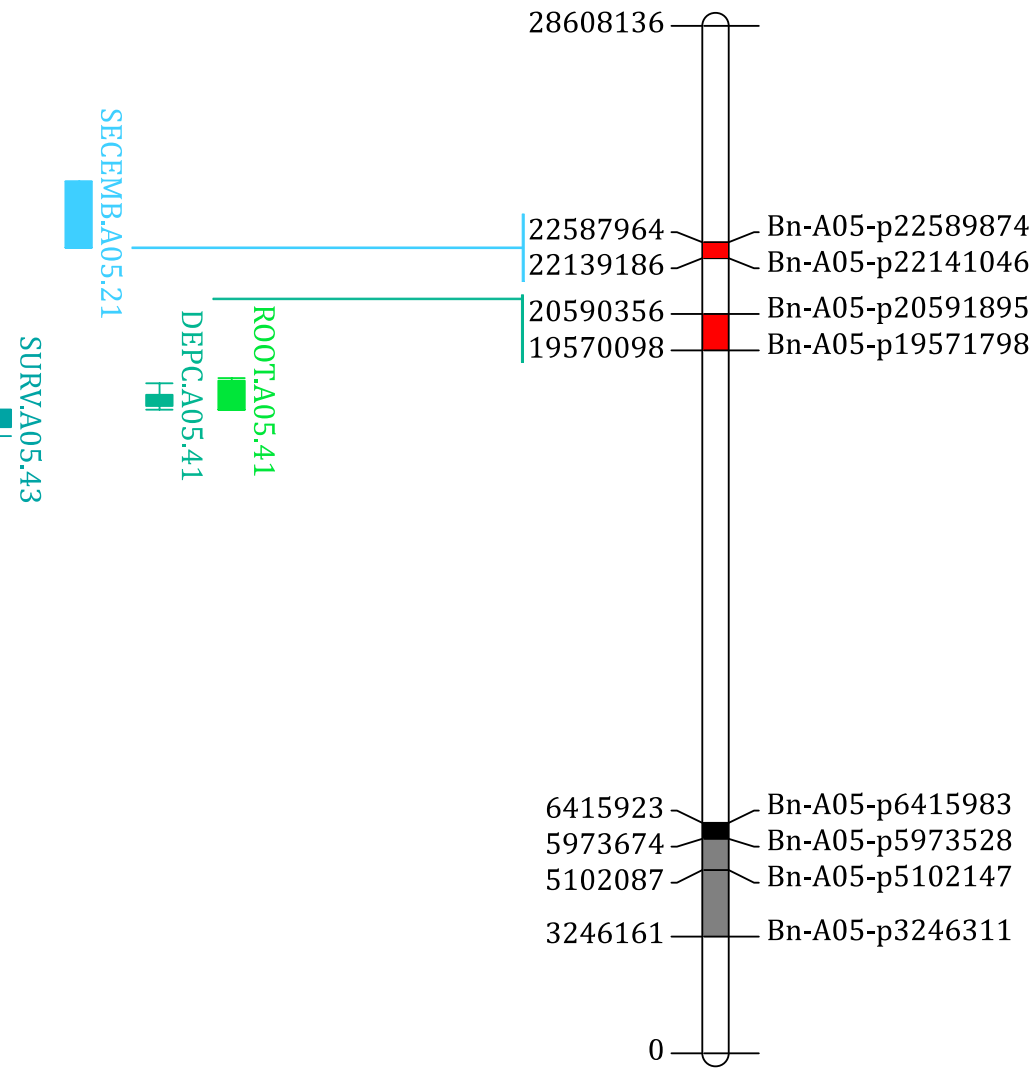

Figure 3.11 QTL positions in linkage group A05 associated with the traits survival (SURV), direct embryo to plant conversion (DEPC) and root (ROOT) regeneration showed overlapping confidence intervals in the genetic map of B. napus. Physical positions in the B. rapa map within the $95 \%$ confidence interval of the QTL accounting for DEPC and SECEMB are shown (red), as well as physical positions of markers flanking putative genomic regions related to microspore embryogenic potential (black) and DEPC (grey) according to Ecke et al. (2015) and Kampouridis et al. (2016).

As previously reported for B. napus, B. oleracea and B. rapa (Zhang and Takahata 2001; Zhang et al. 2003; Sparrow et al. 2004; Holme et al. 2004), and in agreement with the effects of all QTL identified in the present study, in vitro culture response (plant regeneration from protoplast and petioles, androgenesis and direct embryo to plant 
conversion) seems to be under genetic control of loci with additive effects. However, an important contribution of dominant effects and epistatic interactions has been also stated (Zhang and Takahata 2001; Sparrow et al. 2004; Seo et al. 2013), an issue that was not assessed at the DH population DH4079 x Express 617.

Except for the QTL SECEMB.A10.20 for secondary embryogenesis, the QTL alleles increasing the value of all traits are derived from Express 617. This result is consistent with the trait direct embryo to plant conversion since Express 617 is the much better responding parental genotype. However, for microspore embryogenic potential, our findings are opposite to the expected results, taking into account that DH4079 displayed a higher microspore embryogenic potential $(1.4 \%)$ compared to Express 617 (0.003 $\%)$. In order to avoid errors and to confirm the position and direction of the effects, a second QTL analysis was performed using QTL Network 2.1 (Yang et al. 2008), which resulted in a very similar output compare to WinQTL Cartographer 2.5 (Wang et al. 2012). Seo et al. (2013) evaluated the callus and shoot regeneration potential of B. rapa germinated hypocotyls, identifying an unexpected opposite direction of the effects on $50 \%$ of the QTL identified. A possible explanation for this result is the presence of epistatic interactions between loci, a very common fact influencing complex traits (Walley et al. 2012).

It is very important to point out that the mapping population of this research was restricted to $99 \mathrm{DH}$ lines due to the methodological difficulties to determine the microspore embryogenic potential and direct embryo to plant conversion of a satisfactory number of individuals. The size of the population is a critical factor affecting the power of QTL detection since it depends on recombination frequencies. Then, in small populations, the effect of QTL tend to be overestimated, while QTL with small effects are not detected (Zhang et al. 2014a). High-resolution mapping in larger populations has shown that usually single QTL splits in multiple closely linked QTL with often opposite effects (Mackay et al. 2009; Zhao et al. 2005). Even though DH4079 and Express 617 showed contrasting responses for both traits, microspore embryogenic potential and direct embryo to plant conversion, another reason why several QTL could not be detected, may be the absence of polymorphisms between the parental lines in loci influencing such traits. Absence of polymorphic markers may also cause the large gaps observed in linkage groups A07 and C06 (cf. figure 3.5). 


\subsubsection{Candidate Gene Analysis}

In order to identify the genetic factors involved in microspore culture, a BLAST analysis was performed on B. rapa and B. oleracea genome databases to locate physical positions of the QTL for microspore embryogenic potential, secondary embryogenesis and direct embryo to plant conversion. An exhaustive search of candidate genes was performed for the $95 \%$ confidence interval of the QTL. This resulted in the identification of nineteen candidate genes with a strong relation to microspore culture success.

On linkage group A01, the gene Bra028326 collocalized with the QTL POT.A01.67 for the trait microspore embryogenic potential. Bra028326 encodes for the enzyme isopentenyltransferase-7 (IPT7) which is involved in the biosynthetic pathway of cytokinins. Elhiti et al. (2012) identified a notable increase in IPT7 transcripts in androgenic cultures of $B$. napus and its up-regulation has also been observed during the induction period of somatic embryogenesis in zygotic embryos of Arabidopsis lines overexpressing the B. oleracea gene SHOOTMERISTEMLESS (STM). STM encodes a knotted-like homeobox protein required for the formation and maintenance of meristems and its overexpression correlated with an increment on somatic embryogenic potential (Elhiti et al. 2010). The enhancement of isopentenyltransferase7 transcripts may result in an increase of cytokinins which possibly induce microspore embryogenesis due to their ability to control mitosis and cytokinesis, being able to induce cell division and differentiation in in vitro cultures (Zur et al. 2015).

A collocation of the gene Bra037583 and the QTL POT.A01.67 in A01 was also identified. Bra037583 encodes a formylglycinamidine ribonucleotide synthase (PUR4, PURINE BIOSYNTHESIS 4), associated with purine biosynthesis (TAIR 2016). PUR4 function affects male gametophyte development and a significant increase in its expression has been observed when comparing microspore derived embryos with pollen (Joosen et al. 2007). Nevertheless, its possible influence on microspore embryogenic potential has not been established.

The gene Bol016999, on linkage group C06, encoding an oleosin was collocated with the QTL POT.C06.134 associated with microspore embryogenic potential. Oleosin belongs to an embryo specific protein group related to lipid storage which transcript has been 
detected in zygotic, somatic and microspore embryos in B. napus. Oleosin is upregulated on different stages during embryo development (Tsuwamoto et al. 2007; Malik et al. 2007; Malik et al. 2008), and transcripts seem to be accumulated altogether with major storage protein genes, napin and cruciferin (Hays et al. 2001). However, a direct link to microspore embryogenic potential was not identified.

On linkage groups C06 and A05, the genes Bol042965 and Bra008076 collocate with the QTL POT.C06.134 and DEPC.A02.49 respectively. Such QTL were identified for the traits microspore embryogenic potential and direct embryo to plant conversion. Bol042965 encodes CLAVATA3/EMBRYO SURROUNDING REGION-RELATED PEPTIDE 25 (CLE25), while Bra008076 encodes CLAVATA3/EMBRYO SURROUNDING REGION-RELATED PEPTIDE 1 (CLE1), both homologous to the CLAVATA3 (CLV3) gene of $A$. thaliana. CLE25, CLE1 and CLV3 belong to the CLAVATA family of receptor kinase proteins that enhances cell differentiation of peripheral cells of the meristem by repressing the expression of WUSCHEL (WUS), a gene related to apical meristem identity maintenance. CLAVATA1 (CLV1) is implicated in the regulation of the shoot apical meristem establishment. CLV1 mutants show an enlarged area of WUS expression, since a normal pattern of cell division and differentiation is missing, and larger meristems are formed (Schoof et al. 2000). In Arabidopsis ectopically overexpressing the B. napus CLV1 gene, WUS expression decreased and the size of the apical meristem and embryogenic capacity of hypocotyl explants was diminished too (Elhiti et al. 2010). CLAVATA genes are also essential for the maintenance of the apical meristem during all developmental stages for further organogenesis (Clark et al. 1997). Matsuo et al. (2009) reported the up-regulation of CLAVATA3/EMBRYO SURROUNDING REGION-RELATED PEPTIDE 2 (CLE2) during the first days of induction as a result of the overexpression of the Arabidopsis ENHANCER OF SHOOT REGENERATION 1 (ESR1), which is a key factor influencing in vitro shoot regeneration in root explants (Banno et al. 2001). The function of CLE1 and CLE25 is poorly understood but is has been observed that even though CLE1 can rescue loss-of-function clv3-mutant, CLE25 cannot replace CLV3 activity (Ni and Clark 2006). On the other hand, the CLE25 peptide caused a size reduction of the apical meristem in Arabidopsis as CLV1, while CLE1 did not (Kinoshita et al. 2007).

The genes Bra002576 and Bra034855 encode the transcription factor genes WUSCHEL related homebox-2 (WOX2) and WUSCHEL related homebox-5 (WOX5), which 
collocalized with the QTL SECEMB.A05.21 and SECEMB.A10.20, respectively. During embryogenesis, the process to develop shoot and root meristems is relevant for the correct establishment of polarity. The organization is regulated by the WOX transcription factors in the root meristem and the WUSCHEL (WUS) transcription factors in the shoot meristem. Both are mainly expressed in the meristem quiescent center and are required to maintain the structural and functional integrity of the stem cells (Laux et al. 1996; Sarkar et al. 2007). Presumably, WUS transcription factors act by repressing the transcription of response regulators implicated in the differentiation processes determining cell fates during zygotic embryogenesis and plant development (Maraschin 2005; De Smet et al. 2010). In Arabidopsis, ectopically expressing the $B$. napus SHOOTMERISTEMLESS gene, the expression of WUSCHEL (WUS) was enhanced and it has been related to lines with increased embryogenic capacity by the formation of meristematic cells in hypocotyl explants (Elhiti et al. 2010). WOX2 and WOX8 have been detected in the female gamete and zygote, while WOX9 was identified starting from the bicellular stage of zygotic embryos of Arabidopsis. WOX1 has also been identified during the first stages of embryo formation and its expression during heart and torpedo stages is linked to the initiation of vascular primordium of the cotyledons. Then, after the first mitotic divisions of embryos, WOX expression is confined to the quiescent center of the meristem (Haecker 2004), suggesting that cell identity to generate meristems is determined already at the first zygotic division and its maintenance during the embryo development depends also on the expression of WUS in the shoot and WOX in the root (Elhiti et al. 2010; Soriano et al. 2013). The activity of WUS on embryogenesis was confirmed in Arabidopsis gain-of-function mutants which showed an enhanced somatic embryogenic capacity of root explants, promoting the switch from the vegetative to embryonic growth (Zuo et al. 2002).

In such way, while WUS and STM genes maintain the identity of meristematic cells, CLAVATA genes act negatively inducing cell differentiation and preventing the spread of the stem cell population (Laux et al. 1996; Elhiti et al. 2010). The kinase-associated protein phosphatase (KAPP) seem to be a key factor in the WUS-CLV interaction to determine stem cell identity in the root and apical meristems since KAPP binds to CLV receptors and in this way decreases the CLV repression activity on WUS (Carles and Fletcher 2003). KAPP 4.9 fold up-regulation has been identified in Arabidopsis ectopically expressing the B. napus SHOOTMERISTEMLESS gene, which may be an 
evidence of similar molecular factors involved in zygotic and in vitro embryogenesis (Elhiti et al. 2010). In this way, the integrity of the meristems is regulated by a direct interaction of WUS and CLV genes through a positive-negative feedback loop of both, while the expression of WUSCHEL may start the formation of the apical and subapical meristems while CLV1, CLV2 and CLV3 specifies the tissue domains during embryo development (Brand 2000; Boutilier et al. 2005; Malik et al. 2007; Zhang et al. 2014b). WOX and CLE are then required for proper development of embryos (Malik et al. 2007; Elhiti et al. 2012), participating from the earliest stages to establish the apical and basal domains and maintenance of meristem structural and functional integrity, which is certainly associated with the capacity of cells to regenerate a shoot from the apical meristem and a root from the subapical meristem.

Two genes, Bra002894 and Bra002564 encoding a SCARECROW protein and a SCARECROW-like transcription factor-11, collocated with the physical position of the QTL SECEMB.A10.20, which was related to the trait secondary embryogenesis. SCARECROW (SCR) transcription factor family proteins seem to be also responsible for maintaining stem cells in the meristems and like WUS, are mainly expressed in the quiescent center of root meristems during embryo development and under the regulation of SHORTROOT (SHR) transcription factors (Helariutta et al. 2000). SCR gene product controls the periclinal division that originates the cortex and endodermis cells, participating in cell fate to produce and maintain a radial organization in the root (Sabatini 2003). Expression of SCR in rapeseed microspore cultures starts after 3 days of induction and is maintained during embryo development (Custers et al. 2001), while in zygotic embryogenesis of Arabidopsis, SCR expression is observed in very early stages of embryo development (Jenik et al. 2007), suggesting that similar expression patterns of SCR expression to establish radial symmetry are followed in vivo and in vitro (Tsuwamoto and Takahata 2008). In hypocotyl explants of Arabidopsis ectopically expressing the B. napus STM genes that induce somatic embryogenesis, up-regulation of two SCARECROW-LIKE genes was observed (Elhiti et al. 2013).

The genes Bra002859 and Bra008259 collocated with the QTL SECEMB.A10.20 in linkage group A10 and DEPC.A02.49 in A02 respectively. Bra002859 and Bra008259, related to the traits secondary embryogenesis and direct embryo to plant conversion, encode two analogous of the NO-APICAL-MERISTEM (NAM) from Petunia and CUP- 
SHAPED COTYLEDON 3 (CUC3) from Arabidopsis. NAM and CUC belong to NAC domain transcriptional regulator superfamily related to the formation of boundary cells of the meristems, contributing to the formation of the apical meristems and cotyledon detachment (Zimmermann and Werr 2005), shifting from radial to bilateral symmetry of the embryo during the globular-heart stage transition (Zhang et al. 2014b). Petunia nam- and Arabidopsis cuc1- and cuc2- mutants fail to develop shoot meristems, and cotyledons are usually fused, blocking the normal development of the shoot (Souer et al. 1996; Aida et al. 1997; Aida et al. 1999). The NAC activity in the meristem initiation may be related to the SHOOTMERISTEMLESS (STM) gene, whose expression depends on CUC1-3 (Aida et al. 1999; Takada et al. 2001). Development of axillary meristems and shoot and leaf arrangement are also dependent on CUC2 and CUC3 expression (Hibara et al. 2006). Arabidopsis thaliana-CUC3 expression has also been detected in boundaries of lateral roots and trichomes, suggesting that this gene could be related to the establishment of cell lineage boundaries in the root (Xie 2000). Root explants of Arabidopsis overexpressing the ENHANCER OF SHOOT REGENERATION 1 (ESR1) gene, and which expression is positively correlated with the shoot regeneration efficiency, showed up-regulation of CUC1 when transferred to shoot induction medium (Matsuo et al. 2009). Such results indicated that NAC transcriptional regulators are key factors in the shoot apical meristem initiation, primordia position and organ boundary establishment. In such way, NAC transcription factors are required for the correct multicellular organism development and growth of lateral organs of the plant, influencing not only the embryo body but also the direct embryo to plant conversion.

A gene positioned in linkage group A10 within the confidence interval of the QTL SECEMB.A10.20 accounting for secondary embryogenesis, Bra002957, encode the enzyme thiamine-4, responsible for thiamine biosynthesis. Up-regulated thiamine genes have been identified in various stages of embryo development, but with a special transcription enhancement during the first induction days of androgenesis of B. napus (Joosen et al. 2007; Malik et al. 2007; Malik et al. 2008). A pair function in thiamine biosynthesis and mitochondrial DNA damage tolerance has been associated with thiamine enzymes expressed during all seed developmental stages and seedling development stage (TAIR 2016). Thiamine enzymes are involved in the response to oxidative and cold stress, and these functions may be associated with the competence of 
microspores to develop embryos and the ability of such embryos to convert into plants after cold treatment.

Three genes, Bra002994 on A10 which collocated with the QTL SECEMB.A10.20, and Bra022221 and Bra027219 that collocated with the QTL DEPC.A05.41 in linkage group A05, encode for late embryogenesis abundant domain-containing proteins (LEA). In Arabidopsis, somatic and zygotic embryogenesis was accompanied by an up-regulation of LEA genes at the latest developmental stage (Holdsworth et al. 2008; Wickramasuriya and Dunwell 2015), as observed in microspore embryogenesis in $B$. napus (Malik et al. 2007; Tsuwamoto and Takahata 2008; Malik et al. 2008). LEA protein transcripts are accumulated during the maturation phase of zygotic embryos (Saleh and Pages 2003). Increased expression of LEA genes is correlated with the exogenous application of the glutathione inhibitor buthionine sulfoximine (BSO) that accelerated microspore derived embryo maturation and increased the growth and performance of embryos during direct embryo to plant conversion in B. napus (Belmonte et al. 2006; Stasolla et al. 2008). A similar outcome was observed in embryos, where the exogenous application of ABA increased the expression of LEA genes resulting in the successful direct embryo to plant conversion (Haddadi et al. 2008), which supports the association of LEA proteins to such trait, even though LEA function is still unknown.

A gene, Bra008362, encoding the gibberellin 2-oxidase-1, collocates with the QTL DEPC.A02.49 for direct embryo to plant conversion on linkage group A05. Gibberellin 2oxidase-1 (GA2ox1) expression has been detected in mature embryos and its function is associated with gibberellin catabolic process (TAIR 2016). Gibberellins (GAs) are essential plant hormones involved in seed germination, and their regulation is associated with different metabolic pathways, but GA 2-oxidation is considered the major inactivation regulating system in Arabidopsis (Rieu et al. 2008a; Yamaguchi 2008). Therefore, the reduced expression of gibberellin 2-oxidases is correlated with a fast seed germination, while defective GA biosynthesis resulted in a prolonged seed dormancy (Lo et al. 2008). During vegetative and early reproductive phases, AtGA20ox1 and AtGA20ox2 are the mostly expressed gibberellin 2-oxidases and their function is considered to be partially redundant (Rieu et al. 2008b). In Arabidopsis, the loss-of-function deactivation of the gene GA2ox2 caused an increment of GA4 content accompanied by the enhancement of germination during imbibition (Yamauchi et al. 
2007). It was confirmed with a double AtGA20ox1/AtGA20ox2-mutant, which showed more efficient germination when compared to the wild type. Germination of the double mutant was enhanced when a cold treatment was applied (Rieu et al. 2008b). It is well known that gibberellin 2-oxidases inhibit seed germination in the absence of light and cold conditions (Yamaguchi 2008). Cold treatment stimuli have shown to be effective for the direct embryo to plant conversion in B. napus (see Chapter 2). In this way, the expression level of the enzyme gibberellin 2-oxidase-1 could be associated with the further development of plants resulting from microspore derived embryos, acting similarly in in vivo and in vitro systems.

The gene Bra008421 was localized on chromosome A02 within the confidence interval of the QTL DEPC.A02.49 for direct embryo to plant conversion. The gene Bra008421 encodes a phosphoglycerate kinase, an enzyme of the glycolytic pathway catalyzing the transfer of a phosphate group from 1,3-bisphosphoglycerate to ADP to produce ATP (Liu et al. 2015). Phosphoglycerate kinase was up-regulated at various stages of microspore derived embryo development of B. napus (Joosen et al. 2007; Elhiti et al. 2013). In primed oilseed rape seeds, showing an improved germination, an accumulation of phosphoglycerate kinase was observed (Kubala et al. 2015). In Arabidopsis, phosphoglycerate kinase was accumulated before radicle protrusion (Gallardo et al. 2001), and storage lipid mobilization may be required for a correct germination. In this regard, up-regulation of phosphoglycerate kinase in microspore derived embryos may be an important factor related to their direct conversion into plants.

Previous studies assessing gene expression during androgenesis have suggested additional important microspore embryogenesis-related genes for instance BABYBOOM1 (BBM1), ABSCISIC ACID INSENSITIVE3 (ABI3), ARABIDOPSIS THALIANA SEED GENE 1 (ATS1), LEAFY COTYLEDON group (LEC1, LEC2 and (FUSCA3), FAD1, SHOOTMERISTEMLESS (STM), CUP-SHAPED COTYLEDON1 (CUC1) and ZWILLE (Boutilier et al. 2002; Malik et al. 2007; Tsuwamoto and Takahata 2008; Elhiti et al. 2013; Zhang et al. 2014b). The physical positions of those candidate genes did not collocate with the $95 \%$ confidence interval of the QTL identified for microspore embryogenic potential and direct embryo to plant conversion. Nevertheless, very 
important genes previously related to these traits, such as CLE1, CLE25, WOX2, WOX5, SCARECROW, CUC3, NAC and LEA were found.

\subsubsection{Conclusions and Outlook}

The development of doubled haploid plants are of major interest for oilseed rape breeding and has been used since some decades for basic and practical research (Ferrie and Möllers 2011). Microspore culture in oilseed rape is a very important tool whose relevance has increased over the years (Tanhuanpää et al. 1994). Nevertheless, even with very efficient protocols, enormous differences in the microspore embryogenic potential remain among different $B$. napus genotypes, that is indeed restricted by a poor embryo production and a low direct embryo to plant conversion (Chuong et al. 1988), making the development of DH populations still expensive and time-consuming.

Until now, many studies have focused on examining genomic regions with distorted marker segregation in Brassica species that indicate areas enriched with alleles that may confer the ability to generate DH plants. However, distorted marker segregation is a very common phenomenon as previously stated, which is not only influenced by the genotype but also by the environment (e.g. experimental conditions) and random effects (Foisset et al. 1996). In the present research, 6 out of 10 QTL collocated with biased marker segregation areas. Therefore, distorted marker segregation is not a definitive proof of regions controlling microspore culture response, because not all genomic areas related to androgenesis success are identical to regions with distorted marker segregation. A further detailed examination on highly distorted areas (segregation ratio $>1: 3$ ) on linkage groups A01, A03, A08, A09, C03, C04 and C08 could reveal the presence of previously reported candidate genes (e. g. STM, CLV, WUS, WOX) related to microspore culture response.

Microspore culture is a very time-consuming method and exhaustive work is needed to study the microspore embryogenic potential of a mapping population. For this reason, no previous QTL analysis for microspore embryogenesis was performed (Ecke et al. 2015), and the present research represents a pioneer study. 
Further characterization of additional DH lines from the DH4079 x Express 617 mapping population would increase the power of QTL detection and could allow a more reliable identification of genes responsible for microspore embryogenic potential and direct embryo to plant conversion. Additional specific studies should also focus on the QTL hot-spot region on linkage group A05.

Possible candidate genes underlying QTL associated with the microspore embryogenic potential to generate embryos and their ability to convert into plants were proposed. Suggested candidate genes are involved in relevant biosynthetic pathways (e.g. gibberellin catabolic pathway), metabolic processes (e.g. storage product mobilization) and at the transcriptional level (e.g. apical and subapical meristem initiation). Further allele characterization of the suggested candidate gene loci in high- and low-responsive lines from the DH4079 x Express 617 DH population, could provide substantial information about their influence in microspore embryogenic potential and direct embryo to plant conversion. The analysis of different parental allele effects could also facilitate the transfer of the microspore embryogenic potential from responsive to nonresponsive lines.

The development and evaluation of gain- and loss-of-function mutants of candidate genes in B. napus would support their employment as markers to categorize high- and low- responsive genotypes. At the same time, the corroboration of the level and process of influence of such genes may direct the focus on specific experimental microspore culture improvements, either to enhance the ability of microspores to respond to inductive signals and initiate and maintain the embryo development, or to increase the capacity of embryos to convert into plants.

Since heritability values were high and many QTL were identified, it was confirmed that genetic factors are involved in microspore success, which makes feasible the transfer of the embryogenic ability from highly responding to non- or low-responding genotypes. Thus, the microspore culture ability can be genetically improved in Brassicas (Zhang and Takahata 2001). It would be then an important issue to determine whether genes involved in microspore embryogenesis are closely linked to genes of agronomic interest. Previously, skewed segregation in microspore culture was considered a disadvantage for breeding programs (Zhang et al. 2003; Bélanger et al. 2016) because prevalent 
genomic regions could be linked to undesirable agronomic traits (Tanhuanpää et al. 1994). Nevertheless, several studies on DH oilseed rape microspore derived populations have shown that a very broad phenotypic diversity is conserved for most characters of interest: oil quality (Delourme et al. 2006; Qiu et al. 2006; Zhao et al. 2005), fiber and protein content (Suprianto 2014; Zhang et al. 2006), glucosinolate content (Brandes 2014; Uzunova et al. 1995), resistance (Su et al. 2015; Voorrips et al. 1997), phytosterols (Amar et al. 2008; Teh and Möllers 2016) and yield (Lichter et al. 1988; Chen et al. 2007). Considering this, DH plant population development by microspore culture technique is a very suitable tool that complement conventional breeding methods by selecting profitable combinations of alleles (Walley et al. 2012). 


\section{Chapter 4}

Effect of a low temperature treatment on gene expression in microspore derived embryos of Brassica napus $\mathrm{L}$. 


\subsection{Abstract}

The success of microspore culture success is a concern for the production of doubled haploid populations. Since the response to microspore culture is genotype dependent, the contrasting performances of the direct conversion of microspore derived embryos to plants observed in several oilseed rape genotypes makes Brassica napus the perfect model plant to analyze functional genomics involved in the development of microspore derived embryos and its subsequent plant regeneration. Cold pretreatment of embryos has shown to enhance direct embryo to plant conversion and the study of the effect of low temperature on gene expression would give important insights of the genetic factors involved in plant regeneration from microspore derived embryos, which is still considered a constraint of the development of double haploids. In this context, the aim of the present research was to study gene expression profiles through MACE (Massive Analysis of cDNA Ends) of cold-induced embryos derived from the genotypes DH4079 and Express 617. These genotypes show a contrasting direct embryo to plant conversion performance, which is useful to identify differentially modulated genes with a similar expression pattern in both genotypes that is possibly related to the enhancement of direct embryo to plant conversion under low-temperature induction. Microspore culture of the winter oilseed rape genotype Express 617 and the spring genotype DH4079 was performed following a standard protocol. Microspore derived embryos at the late cotyledonary stage were cold treated for 10 days at $2{ }^{\circ} \mathrm{C}$, while control embryos were incubated for 10 days at $20^{\circ} \mathrm{C}$. The procedure was repeated for each genotype. After this, embryos were frozen and MACE was performed by GenXPro GmbH, Frankfurt am Main, Germany. MACE revealed 86,557 different transcripts from which 1,970 genes were exclusively expressed in cold treated embryos of both genotypes. As a whole, 2,606 transcripts exhibited a differential expression (log2FoldChange>2.3) and 207 transcripts were up- or down-regulated in both genotypes in cold treated embryos compared to the control treatment. Of the 207 transcripts, 187 transcripts were annotated to known gene sequences and the top ten up- and down-regulated cDNA sequences were used for BLAST analysis to identify orthologous genes in the B. napus, B. oleracea, B. rapa and A. thaliana databases. Genes involved in plant defense, stress tolerance and cell detoxification were up-regulated (MATE efflux family protein, AP2/ERF transcription factors, Betv1/MLP like protein), as 
well as genes encoding for carbohydrate and lipid transporters (SWEET12 and SRPBCC ligand-binding domain-containing protein), and genes translated into enzymes with hydrolase activity (HAD and END01). Three more genes, BnaC06g15680D, BnaC05g26290D and BnaC07g40880D, encoding B. napus proteins with unknown function were highly up-regulated. Conversely, genes encoding proteins related to stress tolerance with an increasing accumulation during embryo development (M17, LEA49 and glycine-rich protein), were down-regulated in cold-treated embryos. Furthermore, three genes encoding proteins with enzymatic activity (GDSL lipase/esterase and Xyloglucan endotransglucosylase) and a transcription factor (AGL67) were highly down-regulated. Four gene transcripts, BnaC05g11200D, BnaC05g30620D, BnaA01g09770D and BnaC05g05660D, with unknown function were also down-regulated. Results of this study showed that exposure to cold temperature regulates gene expression of microspore derived embryos that could be associated with an enhancement of direct embryo to plant conversion. Further studies on differential gene expression, assessed by MACE and the detection of gene alleles associated to the contrasting response of genotypes, may lead to the identification of candidate genes related to plant regeneration from microspore derived embryos under cold-stress conditions. 


\subsection{Introduction}

Androgenesis is a crucial issue for the production of haploid and doubled haploid individuals. The enormous microspore embryogenic potential of some oilseed rape genotypes and a high sequence similarity of many genes to Arabidopsis thaliana (Brunel et al. 1999; Fourmann et al. 2002) have made Brassica napus a model plant to analyze functional genomics of microspore embryogenesis, replacing in this regard the nonandrogenic A. thaliana (Hosp et al. 2007). Nevertheless, much less attention has been given to the genetic factors involved in the subsequent direct embryo to plant conversion, which is considered a bottleneck for the development of double haploid populations (Möllers and Iqbal 2009).

Pioneer studies on gene expression have shown that a major feature influencing the successful plant regeneration from microspore derived embryos is the proper formation and maintenance of the shoot apical meristem (Belmonte et al. 2006; Stasolla et al. 2008). For instance, the SHOOTMERISTEMLESS (STM), ZWILLE (ZLL), ARGONAUTE1 (AG01), CLAVATA (CLV), WUSCHEL (WUS), and WUSCHEL RELATED HOMEOBOX (WOX) genes have been shown to be involved in the initiation of the shoot and root meristems, as well as in the preservation of stem cell identity and regulation of cell fates in embryo cultures of Brassicas and A. thaliana. The increased expression of the mentioned genes positively regulates CUP-SHAPED COTYLEDON (CUC) and SCARECROW-LIKE genes, which are also involved in cell fate decision (Tsuwamoto and Takahata 2008; Stasolla et al. 2008; Elhiti et al. 2010; Elhiti et al. 2012; Elhiti et al. 2013). Therefore, an increased expression of genes involved in the establishment of an appropriate structure and functionality of the shoot apical meristem may enhance direct embryo to plant conversion.

Since microspore culture response is genotype dependent, very contrasting performances regarding the direct microspore derived embryo to plant conversion under similar culture conditions were observed. This limits the efficiency of the androgenic system for many genotypes (Ferrie and Möllers 2011). However, it has been observed that direct embryo to plant conversion can be very much improved by a short term low-temperature treatment at $1-4{ }^{\circ} \mathrm{C}$ for a period of 3 to 14 days (e. g. Coventry et 
al. 1988; Cegielska-Taras et al. 2002; Zhang et al. 2006). The short term lowtemperature treatment of microspore derived embryos to enhance their direct embryo to plant conversion has shown to be effective in many different genotypes (e. g. Coventry et al. 1988; Cegielska-Taras et al. 2002; Klutschewski 2013). Hence, it can be accepted, that the cold treatment has a genotype independent positive effect on this trait. In general, cold stress decreases the expression of several genes by the direct inhibition of the cell metabolic activity and by a cold-induced osmotic and oxidative stress (Chinnusamy et al. 2007). As a consequence, low-temperature treatment implicates the reprogramming of gene expression and metabolism (Viswanathan and Zhu 2002).

Massive Analysis of cDNA Ends (MACE) is a new RNA sequencing technology developed by GenXpro GmbH, Frankfurt am Main, Germany, which provides sequence information about transcribed genes and transcript abundance. MACE allows a simultaneous genotyping and transcript quantification with a high resolution since only one sequence read is produced from the $3^{\prime}$-end of each polyadenylated transcript and very lowabundant transcripts are identified in comparison to the RNA-Seq method (Zawada et al. 2014; Bojahr et al. 2016). Hence, MACE is a next generation sequencing system that enables a detailed characterization of differentially expressed genes.

The objectives of the present research were to study the effect of a short term low temperature treatment of microspore derived embryos on gene expression profiles of the genotypes DH4079 and Express 617, and to identify differentially expressed genes with a similar expression pattern in both parental lines, which possibly may be related to the enhanced direct embryo to plant conversion. Among the known rapeseed genotypes, the line DH4079 of the Swedish spring cultivar Topas shows an outstanding microspore embryogenic potential, but only a moderate direct embryo to plant conversion (see chapter 3). In contrast, a very low embryo production is obtained from inbred line 617 derived from the winter oilseed rape cultivar Express, but which shows a good direct embryo to plant conversion under comparable conditions (Klutschewski 2013; Ecke et al. 2015, and see Chapter 3). 


\subsection{Materials and Methods}

\subsubsection{Plant material}

Seeds of the winter oilseed rape genotype Express 617 and of the spring genotype DH4079 were cultivated in the greenhouse. Upon bolting, plants were transferred to a growth chamber for their further use as microspore donor plants (Buds of length 2.5 to $3.5 \mathrm{~mm}$ with microspores at the late uninucleate stage were collected and surface sterilized with $1 \%$ calcium hypochlorite solution and Tween-20. Microspore isolation and culture were performed following a standard protocol. For further details on donor plant growth conditions, microspore preparation and culture see Materials and Methods of Chapter 3. Twenty-one days after culture initiation a subsample of 30 microspore derived embryos was transferred to a Petri dish (92 x 16 mm, Sarstedt AG and Co., Nürnbrecht, Germany) with 12 ml LN13-medium (Lichter 1982; www.duchefabiochemie.com, catalog no. N0252). Petri dishes were incubated for 7 days on a shaker (40 rpm) in a culture room $\left(22^{\circ} \mathrm{C}, 12 / 12 \mathrm{~h}\right.$ light/dark, Osram L Lumilux daylight $58 \mathrm{~W}$, with a light intensity of $70 \mu \mathrm{mol} \mathrm{m} \mathrm{m}^{-2} \mathrm{~s}^{-1}$ ). Well differentiated microspore derived embryos at the late cotyledonary stage with a size of 0.7 to $1.0 \mathrm{~cm}$ in length were transferred to plastic boxes $(10.5 \times 8 \times 5 \mathrm{~cm}$, Volume $0.24 \mathrm{~L}$, Huhtamaki $\mathrm{GmbH}$ and Co.KG, Alf, Germany) containing B5-medium supplemented with $0.1 \mathrm{mg} / \mathrm{L}$ gibberellic acid and solidified with 0.9 \% Plant Agar (Gamborg et al. 1968). Eight microspore derived embryos were placed in each plastic box, to give a total of 2 boxes per genotype. One box each from the genotypes Express 617 and DH4079, were incubated for 10 days at $2{ }^{\circ} \mathrm{C}$ in the dark (Cegielska-Taras et al. 2002). The other two boxes with embryos were incubated in a culture room at $22{ }^{\circ} \mathrm{C}$. After 10 days, boxes with cold treated embryos were transferred to the same culture room at $22{ }^{\circ} \mathrm{C}$ and incubated for another 10 days along with non-cold treated embryos. After 20 days in total, the cold and noncold treated embryos of both genotypes were frozen in liquid nitrogen and stored at -80 ${ }^{\circ} \mathrm{C}$. 


\subsubsection{MACE}

To identify genes that are differentially expressed after a cold treatment to induce direct embryo to plant conversion, a Massive Analysis of cDNA Ends (MACE) was performed on treated and control microspore derived embryos. Therefore, bulked embryos from each genotype and each treatment ( 4 bulks of 8 embryos) were used. RNA extraction and MACE was performed at GenXPro GmbH, Frankfurt am Main, Germany. From each bulk a RNA library was generated. Raw read counts were normalized by dividing the number of each transcript through the sum of the total reads of the library and multiplied by $10^{6}$ ((RawCount/LibraryCount)*1.000.000 = tags per million). Transcript distribution along the libraries was plotted in Venn diagrams using the InteractiVenn tool (Heberle et al. 2015).

\subsubsection{Gene annotation and gene expression profiling}

The transcripts were analyzed by GenXPro GmbH, Frankfurt am Main, Germany through BLAST against the B. napus Genome Database (GENOSCOPE; http://www.genoscope.cns.fr/brassicanapus/). Gene Ontology (GO) term enrichment was obtained according to the functional classification of genes of the Gene Ontology Consortium (http://geneontology.org/).

Significant differentially expressed transcripts were determined by paired comparisons between the libraries of the temperature treated against control embryos of each genotype. The following 2 comparisons were made: untreated embryos against coldtreated embryos of genotype Express 617 and untreated embryos against cold-treated embryos of genotype DH4079. A transcript was considered differentially expressed when the fold change of the normalized expression value was at least five-fold increased or decreased, resulting in a log2FoldChange value $\geq 2.3$, with a false discovery rate $(F D R) \leq 0.05$ and an adjusted P-value $\leq 0.05$. $\log 2$ FoldChange was calculated as the log2 value of normalized read counts in untreated embryos subtracted from the log2 value of normalized read counts in the cold-treated embryos (log2FoldChange = $\log 2$ (normalized read counts cold-treated) - $\log 2(($ normalized read counts untreated)). 
Expression patterns of differential expressed transcripts were assessed by a hierarchical clustering using "dendextend" package of R software version 3.2.2 (Galili 2015; R Development Core Team 2016). Top ten up- and down-regulated cDNA sequences were used for BLAST analysis to identify orthologous genes in the B. napus Genome Database (GENOSCOPE; http://www.genoscope.cns.fr/brassicanapus/), $B$. oleracea Genome Database (Bolbase; http://119.97.203.210/bolbase/index.html), B. rapa Genome Database (BRAD; http://brassicadb.org/brad/index.php) and A. thaliana Database (TAIR; http://www.arabidopsis.org/). Gene annotations were obtained from the Swiss-Prot and TrEMBL UniProtKB (http://www.uniprot.org/uniprot/), InterPro (http://www.ebi.ac.uk/interpro/) and TAIR (https://www.arabidopsis.org/) Databases. 


\subsection{Results}

Massive Analysis of cDNA Ends (MACE) was performed to identify differentially expressed genes of embryos after a cold treatment at $2{ }^{\circ} \mathrm{C}$ for 10 days that enhances the direct embryo to plant conversion. Genome-wide gene expression profiling was performed in bulked set of eight control embryos and eight $2{ }^{\circ} \mathrm{C}$-treated embryos from the two genotypes DH4079 and Express 617. The RNA was isolated only 10 days after the cold treatment, because the idea was not to detect direct gene expression differences caused by the cold treatment, but rather to detect long term differences affecting the enhanced direct embryo to shoot conversion. The total number of reads was $28,572,782$, comprising 13,418,891 reads from the two control libraries (untreated embryos) and 15,153,891 from the two cold treated embryo libraries (Table 4.1). $27,285,187$ reads accounted to known gene sequences in reference to $B$. napus, $B$. rapa and B. oleracea genomes, whereas $1,286,377$ reads were uncharacterized. The total number of reads accounted for 86,557 different transcripts.

Table 4.1 Summary of statistics of mRNA reads in the four libraries of B. napus microspore derived embryos.

\begin{tabular}{llrrr}
\hline & & Control & $\begin{array}{c}\text { Cold } \\
\text { Treated }\end{array}$ & Total \\
\hline Number of uncharacterized reads & Express 617 & 325,582 & 336,051 & \\
& DH4079 & 308,421 & 316,323 & \\
& Total & 634,003 & 652,374 & $1,286,377$ \\
& & & & $4.5 \%$ \\
Number of characterized reads & Express 617 & $6,155,654$ & $6,601,050$ & \\
& DH4079 & $6,517,010$ & $8,011,473$ & \\
& Total & $12,672,664$ & $14,612,523$ & $27,285,187$ \\
& & & & $95.5 \%$ \\
& Express 617 & $6,481,515$ & $6,825,788$ & \\
& DH4079 & $6,937,376$ & $8,328,103$ & \\
& Total & $13,418,891$ & $15,153,891$ & $28,572,782$ \\
\hline
\end{tabular}




\subsubsection{Gene expression}

From the 86,557 identified transcripts, 50,630 were expressed in all four libraries (Figure 4.1). Similar numbers of genes, between 67,895 and 70,050, were expressed in the cold treated and untreated embryos of Express 617 and DH4079. Of the 86,557 transcripts 1,970 genes were exclusively expressed in cold treated embryos of both genotypes; and 1,188 genes were only expressed in untreated control embryos

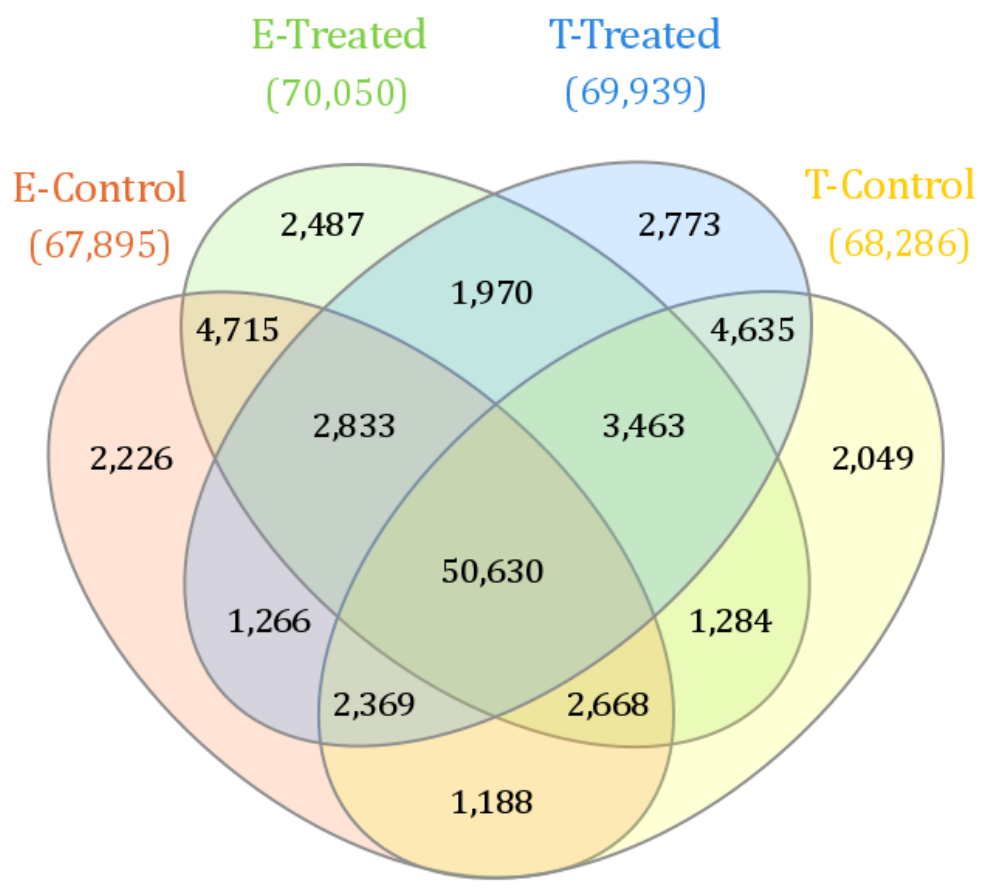

Figure 4.1 Genes expressed in control and cold treated embryos in Express 617 (E) and DH4079 (T) genotypes of B. napus. 50,630 genes were simultaneously detected in each of the four libraries and 1,970 genes were specifically expressed in cold treated embryos of both genotypes.

\subsubsection{Functional classification of genes}

From the 86,557 different entities, 40,997 were assigned to at least one gene ontology (GO) term grouped in the three main functional categories: biological process, molecular function and cellular component (Figure 4.2). The majority of genes $(22,071$, $38.1 \%)$ were assigned to the category molecular function, followed by biological process $(19,256,33.2 \%)$ and cellular component $(16,627,28.7 \%)$. More emphasis was 
made on molecular function and biological process categories since a better understanding of the embryo response to cold treatment could be obtained compared to the cellular component category. The GO terms "binding" (13,787 genes, G0:0005488) and "catalytic activity" (11,765 genes, GO:0003824) were dominant for the main category of molecular function, while "metabolic process" (16,107 genes, G0:0008152) and "cellular process" (12,657, GO:0009987) were dominant for the main term biological process.
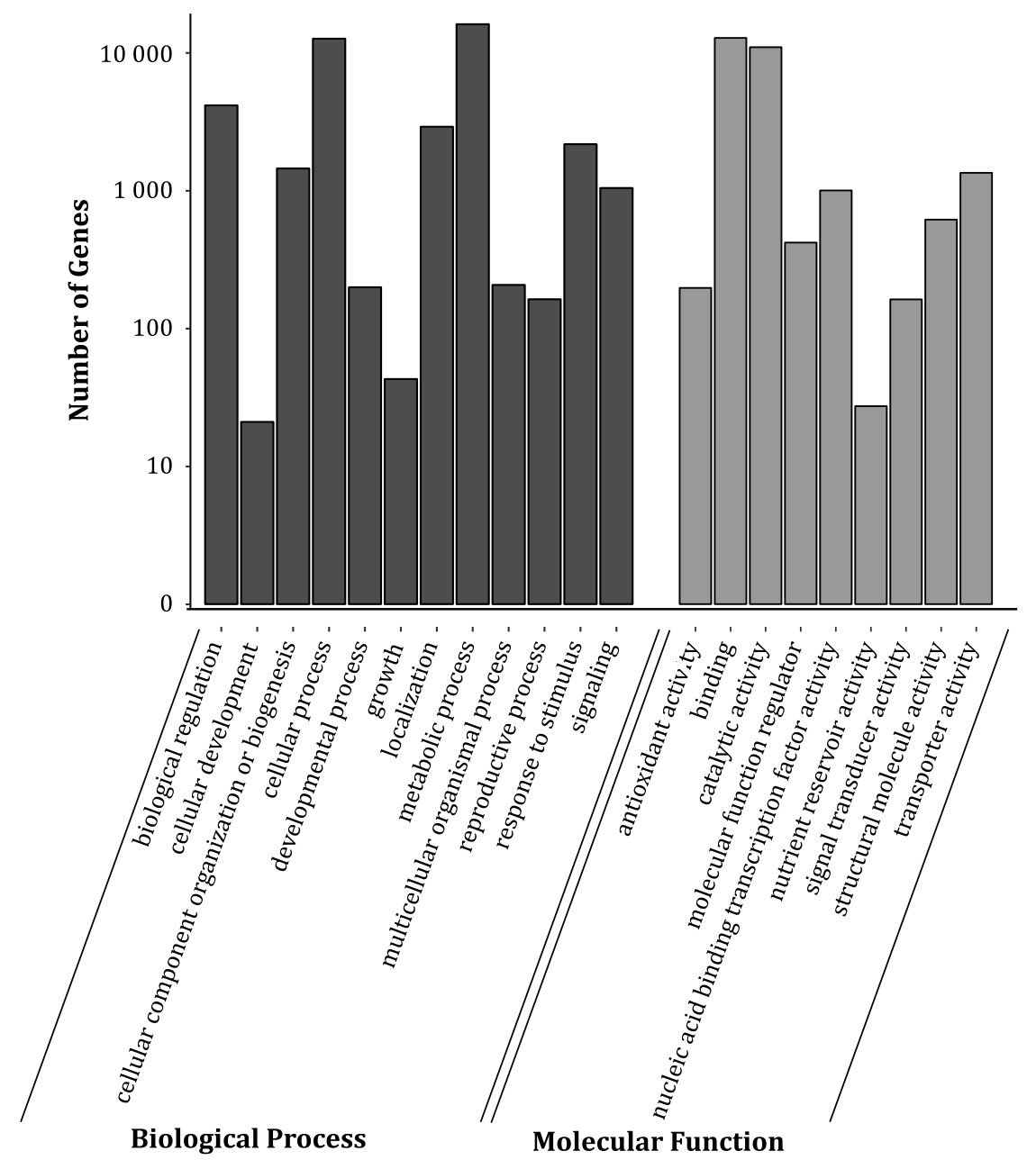

Figure 4.2 Gene ontology (GO) classification of annotated genes in two main GO categories: molecular function and biological process. The number of genes ( $\mathrm{x}$-axis) belonging to the most representative 21 sub-categories (y-axis) are shown. 


\subsubsection{Differentially expressed genes in cold treated embryos of both parental lines}

When analyzing the two parental lines separately, 2,606 transcripts out of the 86,557 exhibited a differential expression with a $\log 2$ ratio $\geq 2.3$ in cold treated embryos compared to the control treatment. Differential expression of the transcripts was visualized by a hierarchical cluster analysis to examine similar and unequal patterns in both genotypes (Figure 4.3). As a result, the expression of 207 (7.9\% of the 2,606 transcripts) transcripts were simultaneously up- or down-regulated in both genotypes, when embryos were cold treated, even when the direction of the regulation was different among the two genotypes for 17 transcripts. From the 207 transcripts, 20 (9.7 \%) corresponded to uncharacterized RNA sequences and 187 (90.3 \%) were annotated to known gene sequences (Figure 4.4). From the annotated transcripts that exhibited a significant dissimilar expression in both genotypes, 67 (35.8 \%) were down-regulated and 120 (64.2 \%) were up-regulated in Express 617. In DH4079, 82 (43.9 \%) transcripts were down-regulated and 105 (56.1\%) transcripts were up-regulated (Figure 4.5b). As a whole, 66 annotated transcripts were down-regulated and 104 transcripts were upregulated in a similar pattern in both parental genotypes.

Only in Express 617, a total of 843 transcripts expressed differentially and 726 (86.1\%) were annotated to gene sequences while 117 (13.9\%) corresponded to uncharacterized RNA. Among the annotated transcripts, 174 (24\%) were down-regulated and 552 (76 \%) up-regulated (Figure 4.5a). In DH4079, the expression of 1,970 transcripts was differentially regulated, from which 236 (12 \%) corresponded to uncharacterized RNA and 1,734 (88\%) matched gene sequences. From the characterized sequences, 873 (50.3\%) were down-regulated and 861 (49.6\%) transcripts were up-regulated in DH4079 (Figure 4.5a). 


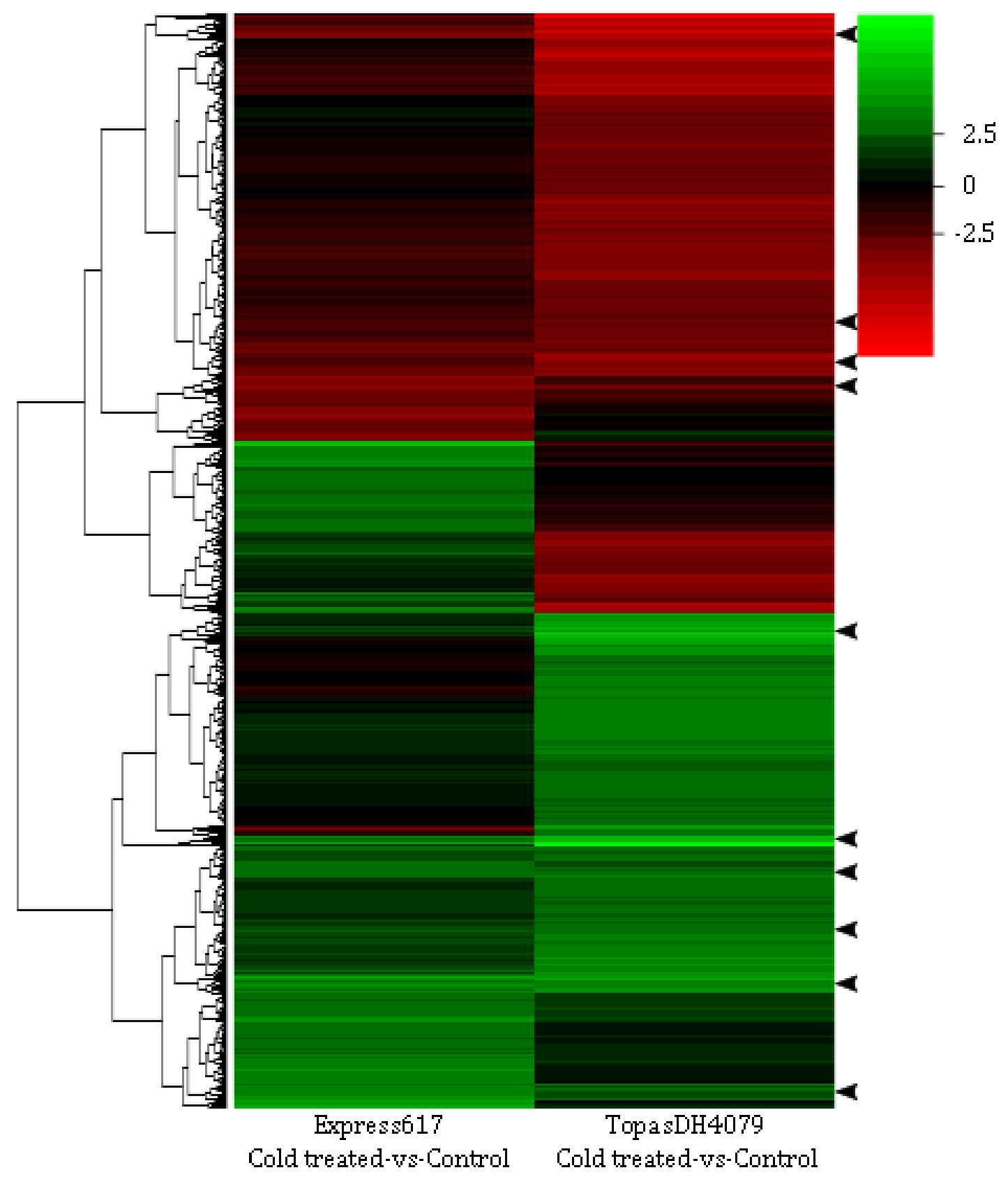

Figure 4.3 Hierarchical cluster analysis of the 2,606 differentially expressed genes in cold treated embryos compared to control embryos from two different rapeseed genotypes, Express 617 and DH4079 (columns). Similar expression patterns in both genotypes are shown (arrows). Hierarchical cluster analysis was based on log2FoldChange values, displaying expression patterns of genes (rows). The expression of a gene was significantly different over a threshold of 2.3 log2FoldChange absolute value. Green palette represents up-regulated expression and red palette represents down-regulated expression. 


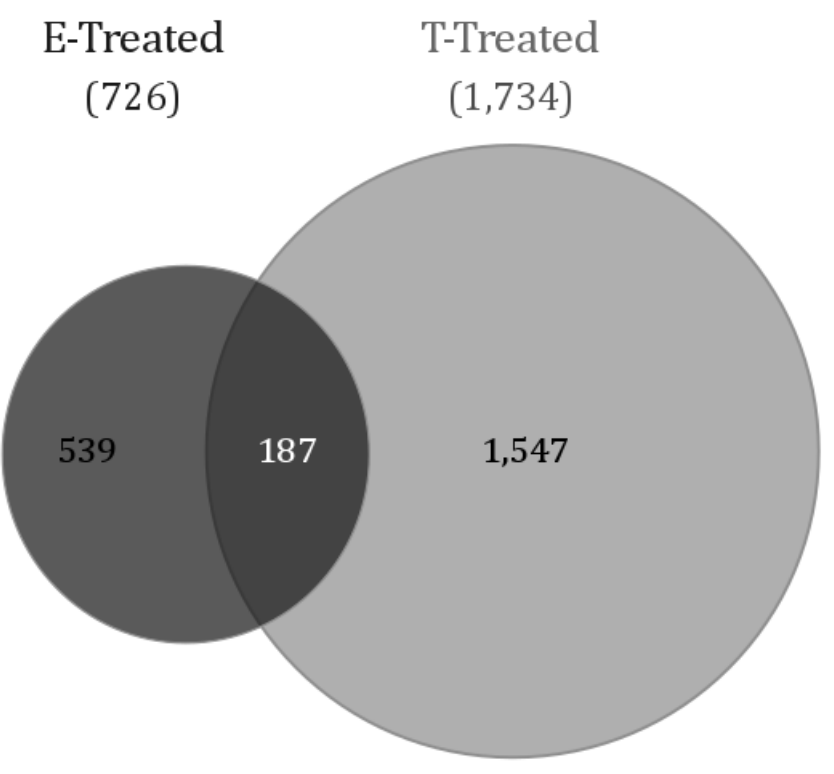

Figure 4.4 Differentially expressed genes ( $>\log 2$ FoldChange 2.3) of cold treated embryos $(p<0.05, \mathrm{FDR}<0.05)$ in comparison to control embryos: 539 genes were exclusively differentially regulated (up- or down- regulated) in Express 617 (E-Treated) and 1,547 in DH4079 (TTreated), whereas 187 genes were differentially expressed in both genotypes.
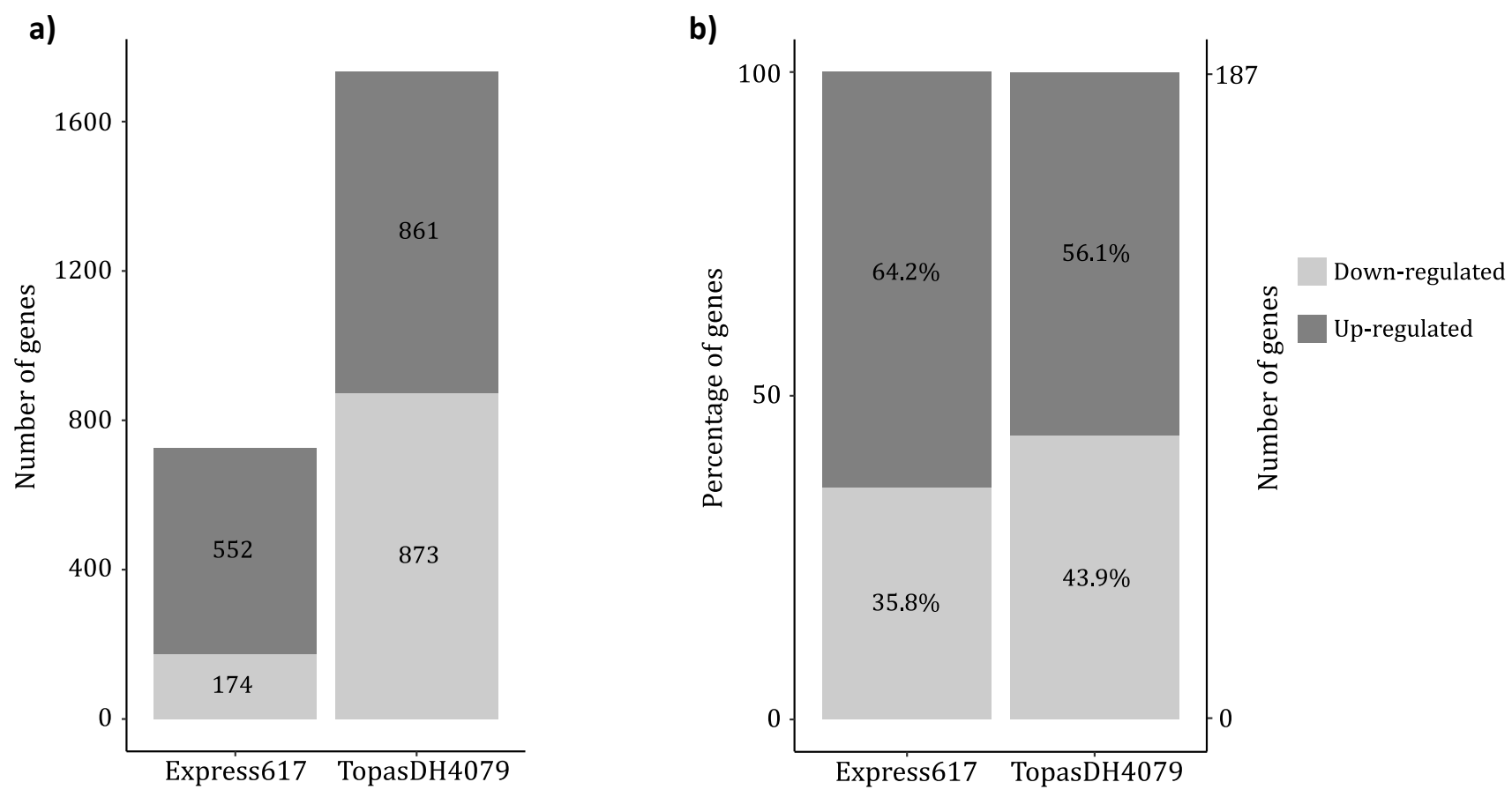

Figure 4.5 Direction of gene regulation in differentially expressed transcripts identified in cold treated embryos in comparison to control embryos in genotypes Express 617 and DH4079. a) A total of 726 genes were differentially expressed in Express 617 and 1,734 in DH4079. b) 187 genes were simultaneously modulated in both genotypes, even though the direction of the gene regulation was not identical in Express 617 and DH4079 for all genes, as revealed by the different percentages shown for the up-and down-regulated expression. 
From the 187 differentially expressed genes in cold treated embryos of both genotypes, the top ten genes showing the highest log2FoldChange in expression were further studied (Tables 4.2 and 4.3).

BnaA09g05190D and BnaA01g11760D were the two most up-regulated genes in cold treated microspore derived embryos (Table 4.2). They encode for integral components of the plasma membrane with transporter activity. The bidirectional sugar transporter SWEET is the protein product of BnaA09g05190D expression, orthologous to SWEET12 in A. thaliana, whereas BnaA01g11760D translates into a Multi Antimicrobial Extrusion (MATE) efflux family protein involved in cell detoxification. A third gene probably involved in transporter activity, BnaC05g01260D, has an A. thaliana orthologue At1g02470, which encodes an SRPBCC (START/RHOalphaC/PITP/Bet v1/CoxG/CalC) ligand-binding domain-containing protein, belonging to the polyketide cyclase/dehydrase and lipid transport superfamily. BnaA09g50010D belongs to the ten top up-regulated genes, which translates into an APETALA2/ETHYLENE RESPONSE FACTOR (AP2/ERF) domain, which belongs to a transcription factor family regulated by ethylene and by abiotic stresses. The orthologue in $A$. thaliana is OCTADECANOIDRESPONSIVE ARABIDOPSIS AP2/ERF 59 (ORA59) involved in plant defense. The upregulated gene BnaAnng33440D encodes for a Betv1/MLP like protein, an orthologue to MLP423 in A. thaliana with a defense-related function. Two genes with enzymatic activity, BnaA05g06860D and BnaA09g47700D, were up-regulated. BnaA05g06860D encodes a Haloacid Dehalogenase (HAD) with hydrolase activity. An A thaliana orthologue was not found. BnaA09g47700D encodes an S1/P1 nuclease, whose orthologue in $A$. thaliana corresponds to the ENDONUCLEASE 1 (ENDO1). BnaC06g15680D was up-regulated and encodes a Yippee family putative zinc-binding protein with unknown function. Another two B. napus genes, BnaC05g26290D and BnaC07g40880D, were up-regulated but the translated proteins have not been characterized.

In the group of the ten top down-regulated genes in cold treated microspore derived embryos, the transcript of BnaA07g33810D with the orthologous AGAMOUS-LIKE 67 transcription factor was found in A. thaliana. The two genes encoding proteins with enzymatic activity, BnaC05g43060D and BnaC09g32760D, were highly down-regulated. BnaC05g43060D encodes a GDSL lipase/esterase, whereas BnaC09g32760D translated 
into a Xyloglucan endotransglucosylase, both with hydrolase activity as their orthologous counterparts in A. thaliana. The A. thaliana orthologous for the downregulated BnaC03g23240D and BnaA03g12360D encode two Late Embryogenesis Abundant (LEA) proteins, M17 and LEA 49, which are related to stress tolerance. The orthologous gene of BnaA09g18220D in A. thaliana encodes a glycine-rich protein also related to dehydration tolerance during cold stress. Four down-regulated gene transcripts, BnaC05g11200D, BnaC05g30620D, BnaA01g09770D and BnaC05g05660D, have not been characterized in B. napus and their function in the orthologous A. thaliana remains unknown. 
Table $\quad 4.2$ Top ten up-regulated transcripts in B. napus cold treated microspore derived embryos of Express 617 and DH4079 genotypes.

\begin{tabular}{|c|c|c|c|c|c|c|c|c|}
\hline \multicolumn{2}{|c|}{ log2FoldChange } & \multicolumn{3}{|c|}{ Best match in B. napus } & \multicolumn{3}{|c|}{ Orthologous genes } & \multirow[b]{2}{*}{ Annotation* } \\
\hline Express 617 & DH4079 & Gene & E-value & ID\% & A. thaliana & B. oleracea & B. rapa & \\
\hline 3.7 & 4.9 & BnaA09g05190D & $1.5 \mathrm{E}-143$ & 98.2 & AT5G23660 & Bo9g014010 & Bra026487 & $\begin{array}{l}\text { SWEET, SWEET sucrose } \\
\text { transporter family proteins }\end{array}$ \\
\hline 3.7 & 5.0 & BnaAnng33440D & 0 & 98.3 & AT1G24020 & Bo7g054680 & Bra012397 & MLP423, MLP like protein \\
\hline 3.7 & 5.9 & BnaC06g15680D & $4.5 \mathrm{E}-131$ & 100 & AT3G55890 & Bo6g069870 & Bra003223 & Yippee-like family protein \\
\hline 3.9 & 6.3 & BnaA01g11760D & $1.3 \mathrm{E}-63$ & 100 & AT4G21903 & Bo1g025740 & Bra013572 & MATE efflux family protein \\
\hline 3.9 & 6.2 & BnaC05g01260D & $9.4 \mathrm{E}-127$ & 98.4 & AT1G02470 & Bo5g003200 & - & $\begin{array}{l}\text { Polyketide cyclase/dehydrase and } \\
\text { lipid transport superfamily protein }\end{array}$ \\
\hline 4.1 & 6.5 & BnaA05g06860D & $3.4 \mathrm{E}-83$ & 100 & & - & Bra005174 & HAD hydrolase \\
\hline 4.2 & 4.9 & BnaA09g47700D & $4.4 \mathrm{E}-48$ & 100 & AT1G11190 & - & Bra031758 & END01, endonuclease \\
\hline 4.4 & 4.1 & BnaA09g50010D & $1.9 \mathrm{E}-152$ & 100 & AT1G06160 & Bo8g114710 & Bra032433 & $\begin{array}{l}\text { ORA59, ethylene-responsive } \\
\text { transcription factor }\end{array}$ \\
\hline 4.5 & 6.8 & BnaC05g26290D & $4.8 \mathrm{E}-144$ & 100 & AT1G49800 & Bo5g076470 & - & BnaC05g26290D protein \\
\hline 4.8 & 4.3 & BnaC07g40880D & $1.4 \mathrm{E}-97$ & 100 & - & Bo7g111410 & - & BnaC07g40880D protein \\
\hline
\end{tabular}

* Gene descriptions were obtained from TAIR ID\% of what to what on RNA sequence level? 
Table 4.3 Top ten down-regulated transcripts in B. napus cold treated microspore derived embryos of Express 617 and DH4079 genotypes.

\begin{tabular}{|c|c|c|c|c|c|c|c|c|}
\hline \multicolumn{2}{|c|}{ log2FoldChange } & \multicolumn{3}{|c|}{ Best match in B. napus } & \multicolumn{3}{|c|}{ Orthologous genes } & \multirow[b]{2}{*}{ Annotation* } \\
\hline Express 617 & DH4079 & Gene & E-value & ID\% & A. thaliana & B. oleracea & B. rapa & \\
\hline-3.5 & -5.3 & BnaC05g11200D & 0 & 98.7 & AT3G21380 & Bo5g020350 & Bra 026177 & $\begin{array}{l}\text { JAL36, mannose-binding lectin } \\
\text { superfamily protein }\end{array}$ \\
\hline-3.4 & -4.2 & BnaC05g30620D & $4.9 \mathrm{E}-28$ & 89.2 & АT3G21480 & Bo5g101000 & Bra031286 & $\begin{array}{l}\text { BRCT domain-containing DNA } \\
\text { repair protein }\end{array}$ \\
\hline-3.2 & -5.2 & BnaA07g33810D & $1.1 \mathrm{E}-130$ & 100 & AT1G77950 & - & Bra015645 & $\begin{array}{l}\text { AGL67, MADSbox family } \\
\text { transcription factor }\end{array}$ \\
\hline-3.2 & -4.7 & BnaA01g09770D & 0.49 & 95.2 & AT4G18975 & - & Bra013365 & Uncharacterized protein \\
\hline-3.2 & -6.1 & BnaC05g43060D & $6.2 \mathrm{E}-119$ & 100 & AT3G09930 & Bo5g137850 & - & GDSL esterase/lipase \\
\hline-3.1 & -6.9 & BnaC09g32760D & $2.9 \mathrm{E}-70$ & 100 & AT5G57550 & Bo9g133240 & Bra002722 & XTH25, glycoside hydrolase family \\
\hline-3.0 & -4.6 & BnaC03g23240D & 0 & 100 & AT2G41260 & Bo3g035510 & - & $\begin{array}{l}\text { M17, late embryogenesis abundant } \\
\text { protein }\end{array}$ \\
\hline-2.9 & -4.6 & BnaA09g18220D & 0 & 100 & AT2G05580 & Bo9g069430 & - & Glycine-rich protein family \\
\hline-2.9 & -4.9 & BnaA03g12360D & $1.1 \mathrm{E}-163$ & 100 & AT5G53260 & Bo3g022820 & Bra029085 & $\begin{array}{l}\text { LEA49, late embryogenesis } \\
\text { abundant protein }\end{array}$ \\
\hline-2.8 & -4.3 & BnaC05g05660D & $2.6 \mathrm{E}-40$ & 100 & AT1G07985 & - & Bra018667 & Uncharacterized protein \\
\hline
\end{tabular}

* Gene descriptions were obtained from TAIR 


\subsection{Discussion}

A major constraint to the development of DH populations through microspore culture is the efficient plant regeneration from microspore derived embryos, that is enhanced by the application of a short cold pretreatment (e. g. Coventry et al. 1988; Cegielska-Taras et al. 2002). The exposure to cold temperature is a stressful system that consequently reprograms the expression of multiple genes (Viswanathan and Zhu 2002). In this research, the expression profiles of cold treated and not cold treated embryos were assessed in order to find putative genes influencing cold-induced direct embryo to plant conversion. The study was complemented by the inclusion of two genotypes, DH4079 and Express 617, which showed a different performance for this trait.

\subsubsection{Gene Expression and Functional Classification}

In a first approach, 86,557 different transcripts were identified through MACE analysis. However, only 50,630 were detected in all of the four libraries, and 1,970 were exclusively expressed in cold treated embryos of both genotypes, showing that the gene expression may be regulated by low temperature.

When transcripts were classified according to their biological function, the most representative Gene Ontology (GO) terms were metabolic and cellular processes followed by response to stimulus. However, the most dominant GO terms for the molecular function category were binding and catalytic activity, in which antioxidant activity was also highly represented. This pattern was observed in the expression profiles of developing zygotic embryos of B. rapa (Zhang et al. 2014b), suggesting a high transcriptional and metabolic activity as well as the activation of a protective mechanism against environmental stress during embryo maturation and conversion into plants.

\subsubsection{Differential genes expression profiling}

In total, 207 transcripts were either up- or down-regulated in both genotypes when embryos were cold treated, from which 187 were annotated to known gene sequences. Of the 187 transcripts, $64 \%$ and $56 \%$ were up-regulated in Express 617 and DH4079, 
respectively. Therefore, the direction of the gene regulation was not equal for all genes in both genotypes, but such higher percentages of up-regulated genes could be an indication of an increase in gene expression resulting from the cold treatment. Similarities in gene expression patterns of cold treated embryos in both genotypes can be then associated with their response to low temperature that enhances direct embryo to plant conversion.

To study in detail the effect of cold treatment on the expression of important genes possibly influencing direct embryo to plant conversion, the top ten up- and downregulated transcripts in both Express 617 and DH4079 were further evaluated.

The gene BnaA09g05190D (Table 4.2) was highly up-regulated and encodes a bidirectional sugar transporter member of the SWEET family, whose orthologue in A. thaliana, SWEET12, is responsible for carbohydrate transport particularly expressed in the phloem (Chen et al. 2012). The SWEET family protein members are expressed at several stages of embryo development and seedlings and have been related to an improvement in seed yield in A. thaliana (Schmid et al. 2005; Chen et al. 2015). The bidirectional sugar transporter SWEET15 was highly up-regulated in a prior germination phase after PEG 6000 mediated osmopriming in B. napus that resulted in an increase of germinated seeds (Kubala et al. 2015). Therefore, SWEET transporters have been involved not only in seed maturation but also in ending seed dormancy (TAIR 2016) that could influence direct plant conversion of microspore derived embryos.

BnaA01g11760D translates into the DETOXIFICATION protein that is a member of the Multi Antimicrobial Extrusion (MATE) efflux family that mediate resistance to several toxins (UniProt 2015). In B. napus, the repression of SHOOTMERISTEMLESS (STM) expression resulted in the down-regulation of MATE efflux genes during microspore embryogenesis (Elhiti et al. 2012). STM is responsible for the proper formation and maintenance of the shoot apical meristem and has been related to an enhancement of embryo yield and improved direct embryo to plant conversion (Elhiti et al. 2013). In the present study, DETOXIFICATION gene was up-regulated in cold-treated microspore derived embryos, which is in concordance with previous research on seed germination, in which a MATE efflux family protein was up-regulated after PEG 6000 mediated 
osmopriming of B. napus seeds prior to germination and its expression was enhanced in 24 h-imbibed seeds of $A$. thaliana (Nakabayashi et al. 2005; Kubala et al. 2015).

BnaA09g50010D was part of the ten top up-regulated genes and encodes an APETALA2/ETHYLENE RESPONSE FACTOR (AP2/ERF) domain (UniProt 2015), which belongs to the transcription factor AP2/ERF family regulated by ethylene and biotic and abiotic stresses via ETHYLENE-INSENSITIVE2 (EIN2) (Fujimoto 2000). AP2/ERF family proteins are expressed in $A$. thaliana at the four- to twelve-leaves stage and its expression has also been associated with cold and oxidative stress response and tolerance in B. napus and A. thaliana seedlings (Schmid et al. 2005; Du et al. 2016; Lv et al. 2016). The A. thaliana AP2/ERF 59 (ORA59) is involved in plant defense and is an essential integrator of the signal transduction pathways that involve jasmonic acid and ethylene. Consistent with the present research, some ERF were up-regulated after PEG 6000 mediated B. napus seed osmopriming prior to germination and in microspore derived embryo shoot meristems of oilseed rape overexpressing STM (Elhiti et al. 2013; Kubala et al. 2015). It has been shown before that the AP2/ERF transcription factors diminish the expression of AGAMOUS in A. thaliana (Drews et al. 1991). This result is consistent with the outcome of the present research, since the $B$. napus gene, BnaA07g33810D, orthologous of the transcription factor AGAMOUS-LIKE 67 (AGL67) in A. thaliana, was down-regulated in cold treated embryos. This result is also supported by the findings of Elhiti et al. (2013), because a decreased STM expression caused an upregulation of AGAMOUS-like TF in shoot meristems of B. napus embryos.

The up-regulated gene BnaC05g01260D is probably involved in the mobilization of storage products during germination since it is orthologous in A. thaliana. At1g02470, encodes an SRPBCC ligand-binding domain-containing protein with lipid transport activity (TAIR 2016), and expression in A. thaliana embryos and up-regulation upon PEG 6000 mediated osmopriming of B. napus seeds prior to germination has been observed (Schmid et al. 2005; Kubala et al. 2015).

The genes BnaA05g06860D and BnaA09g47700D encode a HAD and an S1/P1 nuclease, respectively. Both genes with hydrolase activity were up-regulated as well as the BnaC06g15680D gene that encodes a Yippee family putative zinc-binding protein with unknown function (Mitchell et al. 2015; UniProt 2015). A plant defense-related gene 
that was up-regulated in cold-treated embryos was BnaAnng33440D that encodes for a Betv1/MLP like protein with unknown function (Wen et al. 1997). Betv1/MLP like gene expresses in mature and imbibed seeds and seedlings of $A$. thaliana following application of biotic and abiotic stress (Giavalisco et al. 2005; Nakabayashi et al. 2005; Schmid et al. 2005). Another two B. napus genes, BnaC05g26290D and BnaC07g40880D which encode for proteins are not yet characterized. They were up-regulated in coldtreated oilseed rape microspore derived embryos. An up-regulation of those genes following cold treatment of embryos and its relation to direct embryo to plant conversion has not been reported.

On the other hand, BnaC03g23240D and BnaA03g12360D belong to the group of downregulated genes. The A. thaliana orthologous gene encode two Late Embryogenesis Abundant (LEA) proteins, M17 and LEA 49 (UniProt 2015). LEA proteins are considered cold responsive proteins with an important role in stress protection and desiccation tolerance (Yuan et al. 2012; Candat et al. 2014), and have been involved in embryo development since they are highly up-regulated during embryo maturation in $A$. thaliana, B. oleracea, B. rapa and B. napus (Raynal et al. 1999; Li et al. 2005; Soeda et al. 2005; Zhang et al. 2014b). However, in agreement with the results of the present study, LEA genes showed a decreased expression through seed priming and imbibition and disappeared during germination (Raynal et al. 1999; Soeda et al. 2005; Ge et al. 2013; Kubala et al. 2015).

The $B$. napus gene C09g32760D, which translates into a XYLOGLUCAN ENDOTRANSGLYCOSYLASE/HYDROLASE (XTHs), was down-regulated in cold-treated microspore derived embryos. Consistent with this, Elhiti et al. (2012 and 2013) reported the down-regulation of XYLOGLUCAN ENDOTRANSGLYCOSYLASE 6 and 9, and other genes belonging to the cell wall category in shoot apical meristems of microspore derived embryos overexpressing the B. napus STM gene, that also resulted in the enhancement of direct embryo to plant conversion. Such observation seems contrary to what is expected since XTHs are involved in the modification of primary plant cell wall structure, cleaving xyloglucan polymers in growing tissues (Hyodo et al. 2003).

The transcripts of the BnaC05g43060D and BnaA09g18220D genes were also downregulated. BnaC05g43060D encodes a GDSL lipase/esterase with hydrolase activity as 
its orthologous counterpart in A. thaliana (Mitchell et al. 2015), whereas the orthologous in A. thaliana of BnaA09g18220D translates into a glycine-rich protein that is probably related to cold stress tolerance (TAIR 2016). Four down-regulated gene transcripts, BnaC05g11200D, BnaC05g30620D, BnaA01g09770D and BnaC05g05660D have not been characterized in B. napus; therefore, their function is still unknown. The relationship of the diminished expression of those genes to cold-induced direct embryo to plant conversion was not established.

\subsubsection{Conclusions and Outlook}

The outcome of this study indicated that cold stress modulates gene expression of microspore derived embryos. Promising MACE results on differential gene expression suggested that this procedure could be applied to identify candidate genes related to plant regeneration from microspore derived embryos following cold-stress treatment.

The expression of certain genes responsible for the shoot apical and root subapical meristems initiation and maintenance, such as SHOOTMERISTEMLESS, ZWILLE, ARGONAUTE1, CLAVATA, WUSCHEL and WUSCHEL RELATED HOMEOBOX (WOX), has previously been related to direct embryo to plant conversion success (e. g. Stasolla et al. 2008; Elhiti et al. 2010). A further more comprehensive BLAST analysis of all expressed genes against B. oleracea, B. rapa and $A$. thaliana databases would allow to identify $B$. napus orthologous genes and analyze their possible differential expression associated to a direct embryo to plant conversion enhancement in cold-induced embryos.

Supplementary experimental biological replications with the inclusion of additional low- and high-responsive genotypes would allow a more precise detection of SNP polymorphisms to establish a relation of dissimilar gene alleles to differential response of genotypes.

A comprehensive study of clusters of genes with similar expression patterns as well as a pathway-based analysis (Kyoto Encyclopedia of Genes and Genomes; http://www.genome.jp/kegg/) could increase the understanding of gene interactions and biological processes involved in cold-induced direct embryo to plant conversion. 


\section{Chapter 5}

\section{General Discussion}

Microspore culture is a routinely employed technic for the development of double haploid populations for breeding programs. Despite the progress achieved in the improvement of microspore culture methods, many species remain recalcitrant (Ferrie and Möllers 2011) and the development of successful protocols is much more expensive compared to conventional breeding (Haddadi et al. 2008). Because of that reason, there is an interest to study the genetic basis underlying microspore culture response and the development of molecular markers for this purpose may be useful in breeding programs. Among Brassicas, B. napus is considered to be the most embryogenic species (Ferrie 2003) and remarkable differences among oilseed rape genotypes have been reported (Ferrie and Keller 2007). This makes oilseed rape an outstanding model plant to study functional genetics of androgenesis and zygotic embryogenesis (Custers et al. 2001; Touraev et al. 2001). Therefore, a DH population derived from the cross between DH4079 and Express 617, which show very contrasting responses in their microspore embryogenic potential and direct embryo to plant conversion, was assessed in this study.

\subsection{Genetic Variation in microspore culture response of the DH4079 x Express Population}

Large and significant differences among the genotypes were observed for the traits microspore density, microspore embryogenic potential, embryo survival, direct embryo to plant conversion, root regeneration and secondary embryogenesis. In agreement, a huge variety in the microspore embryogenic potential and direct embryo to plant conversion has been observed among rapeseed genotypes (e. g. Trifonova and 
Atanassov 1997; Babbar and Agarwal 2004; Smýkalová et al. 2006; Klutschewski 2013). Analysis of variance showed a highly predominant genotypic effect for microspore embryogenic potential, direct embryo to plant conversion and related traits. Consequently, high broad-sense heritabilities were observed in the DH4079 x Express $617 \mathrm{DH}$ population, as reported in previous studies in oilseed rape (Zhang and Takahata 2001; Klutschewski 2013; Seo et al. 2013). The major importance of genetic factors involved in microspore culture response indicates that selection of successful genotypes is plausible, and microspore embryogenic potential and direct embryo to plant conversion can be genetically improved. This implies that routinely applying the microspore culture technic in commercial breeding programs will gradually improve tissue culture ability of the breeding material. The question remains if the loci responsible for distorted marker segregation are closely link to agronomic interest. This may be positive or negative depending on the direction of the effect of the allele. However, to date there is no evidence for any positive or negative effect of the androgenic process on the phenotypic trait variation within a segregating population.

\subsection{Distorted segregation and identification of QTL for microspore culture response}

Distorted segregation was identified in $49 \%$ of the SNP markers, which is a common result in double haploid microspore derived populations that show an excess of alleles mostly in favor of the responsive parental genotype (for a review see Ferrie and Möllers 2011). In the DH4079 x Express 617 population $63.4 \%$ of the markers were biased in favor of DH4079 and $36.6 \%$ towards the genotype Express 617, indicating that alleles positively affecting microspore embryogenic potential derived from both parents. A detailed analysis of highly distorted areas (segregation ratio>1:3) located in linkage groups A01, A03, A08, A09, C03, C04 and C08 would enable the possible identification of candidate genes previously associated with microspore culture response, such as BABYBOOM1 (BBM1), ABSCISIC ACID INSENSITIVE3 (ABI3), ARABIDOPSIS THALIANA SEED GENE 1 (ATS1), LEAFY COTYLEDON group (LEC1, LEC2 and FUSCA3), FAD1, SHOOTMERISTEMLESS (STM), CUP-SHAPED COTYLEDON1 (CUC1) and ZWILLE (Boutilier et al. 2002; Malik et al. 2007; Tsuwamoto and Takahata 2008; Elhiti et al. 2013; Zhang et al. 2014b). 
In the present research, 10 QTL related to microspore culture response were detected, from which 6 collocated with areas showing distorted marker segregation. From the 10 QTL, three accounted for microspore embryogenic potential, one for survival of the embryos, and two each for secondary embryogenesis, direct embryo to plant conversion and for root regeneration. QTL for microspore embryogenic potential and direct embryo to plant conversion were located on chromosomes A01, A02, A05, A10 and C06, which is consistent with Ecke et al. (2015) and Kampouridis et al. (2016) findings. Nevertheless, physical positions of QTL and putative genomic regions related to microspore embryogenic potential and direct embryo to plant conversion detected by Ecke et al. (2015) and Kampouridis et al. (2016) did not overlap, which suggests the presence of several different loci controlling androgenesis of B. napus. A further characterization of additional DH lines from the DH4079 x Express 617 mapping population may lead to a more comprehensive detection of QTL since the size of the mapping population in this research was limited to 99 DH lines. Significant and high correlation coefficients $\left(r_{s}=0.66-0.78, P<0.01\right)$ among embryo survival, direct embryo to plant conversion and root regeneration, as well as collocation of QTL of those traits on linkage group A05, pointed out the presence of a clustered region possibly carrying genes responsible for the direct embryo to plant conversion or the existence of a gene with a pleiotropic effect. A detailed analysis of parental alleles in this area, comparing bulked groups of low-responsive genotypes and of high-responsive genotypes for the trait direct embryo to plant conversion could allow the identification of SNP markers useful for further marker assisted selection.

\subsection{Candidate Gene Analysis}

A detailed examination of genes located in the $95 \%$ confidence interval of QTL for the traits microspore embryogenic potential, secondary embryogenesis and direct embryo to plant conversion, resulted in the identification of nineteen putative candidate genes, of which 8 showed a strong relationship to microspore culture response: CLE1, CLE25, WOX2, WOX5, SCR, SCL11, CUC3 and NAC. All these genes are involved in the formation and maintenance of shoot and root meristems. Proper morphology and function of the shoot apical meristem has proven to be of relevance for androgenesis, influencing positively direct embryo to plant conversion (Stasolla et al. 2008; Elhiti et al. 2013). While WOX, SCR, SCR11 preserve the identity of meristematic cells (e. g. Laux et al. 
1996; Schoof et al. 2000; Matsuo et al. 2009; Elhiti et al. 2010), CLV, CUC and NAC genes induce cell differentiation and primordia appearance (e. g. Aida et al. 1999; Takada et al. 2001; Hibara et al. 2006; Matsuo et al. 2009). LEA genes, highly up-regulated during embryo development, as well as hormone metabolism-related genes (IPT7 and GA20ox1) did collocate with QTL for microspore embryogenic potential, secondary embryogenesis and direct embryo to plant conversion. A further evaluation of the allelic variants of such genes present in the parental genotypes, as well as in DH lines showing contrasting responses, could confirm their influence on microspore embryogenic potential and direct embryo to plant conversion. Since a significant reprogramming of gene expression in microspores is needed to switch from the usual gametophytic development to an embryogenic development, a complex genetic network must be involved in the androgenetic process.

\subsection{Expression Profile Analysis}

A gene expression study through MACE was performed in cold-treated microspore derived embryos in comparison to non-treated embryos since a ten-day period of a cold shock has shown to enhance direct embryo to plant conversion (Cegielska-Taras et al. 2002; Klutschewski 2013). MACE revealed 86,557 different transcripts from which 1,970 genes were exclusively expressed in cold treated embryos of both genotypes and 207 exhibited a differential expression (log2FoldChange $>2.3$ ) compared to control treatment. This result revealed that low temperature enhances direct embryo to plant conversion by reprogramming the expression of genes involved in several processes, such as plant defense, stress tolerance and cell detoxification that were part of the 10 most up-regulated genes (MATE efflux family protein, AP2/ERF transcription factors, Betv1/MLP like protein). AP2/ERF is a family of transcription factors with a regulatory role on plant response to biotic and abiotic stresses (Du et al. 2016). BABY BOOM gene, which has an important role in triggering the embryogenic growth on somatic tissues (Boutilier et al. 2002), and ESR1 (Enhancer of Shoot Regeneration 1) gene whose overexpression increased in vitro shoot regeneration (Banno et al. 2001; Matsuo et al. 2009), belong to the AP2-ERF family. BABY BOOM gene along with several other AP2ERF members, displayed an outstanding differential expression during Brassica androgenesis (e. g. Tsuwamoto et al. 2007; Zhang et al. 2014), pointing out the relevance of the AP2-ERF family during microspore culture response. A strong up- 
regulation also occurred for genes encoding carbohydrate and lipid transporters (SWEET12 and SRPBCC ligand-binding domain-containing protein), possibly related to storage product mobilization during germination and seedling establishment (Schmid et al. 2005; TAIR 2016). On the other hand, genes related to stress tolerance (M17, LEA49 and glycine-rich protein) belong to the group of the most down-regulated ones in coldtreated embryos. LEA and glycine-rich proteins have been associated with embryo maturation, accumulating during embryo development, but decreasing during seed germination (Raynal et al. 1999; Soeda et al. 2005; Ge et al. 2013; Kubala et al. 2015). Results of this study showed that the exposure of microspore derived embryos to cold temperature regulates gene expression that could be associated with an enhancement of direct embryo to plant conversion. Further replicated expression profiles and the SNP characterization of highly up- and down-regulated transcripts may allow the identification of candidate genes for improved plantlet regeneration induced by cold treatment. A comparison of gene expression profiles through MACE along the complete process of direct embryo to plant conversion, namely, from the onset of the coldtreatment until the appearance of the shoot, would increase the knowledge of the genetic factors involved in every of the several stages involved in plantlet regeneration.

\subsection{Conclusion}

The outcome of this study showed a large variation on microspore embryogenic potential and direct embryo to plant conversion in the DH4079 x Express 617 population with high heritability values, which pointed out the predominantly influence of genotypic factors on such traits. QTL and putative candidate gene detection make plausible the identification of high responsive genotypes and the transfer of alleles positively affecting microspore culture efficiency to low-responsive genotypes during plant breeding programs for the development of double haploid populations. The employment of recent sequencing and genotyping tools as MACE would lead to an earlier recognition of genes directly involved in microspore culture response. 


\section{Chapter 6}

\section{Summary}

Microspore culture is a very powerful technique in breeding of oilseed rape for the rapid and efficient generation of completely homozygous doubled haploid (DH) lines. Despite the progress achieved in optimizing tissue culture protocols, tremendous differences remain among Brassica napus genotypes in their embryogenic response and direct embryo to plant conversion, representing a hindrance in the development of double haploid populations for plant breeding purposes.

To understand the genetic factors underlying microspore culture response, the following objectives were addressed: (a) to develop a doubled haploid population from the cross of the highly embryogenic line DH4079 and the low embryogenic inbred line Express 617; (2) to characterize the doubled haploid population for its microspore embryogenic potential, its direct embryo to plant conversion as well as for microspore density, embryo survival, root regeneration and secondary embryogenesis; (3) to develop an Illumina SNP-chip based molecular marker map, to identify QTL for those traits and to localize candidate genes within QTL confidence intervals for the traits of interest; and (4) to study the gene expression pattern in low-temperature treated microspore derived embryos with the aim to identify differentially expressed genes which may be involved in the enhanced direct embryo to plant conversion after cold induction.

In vitro propagated F1-plants of the cross DH4079 x Express 617 were used to generate a DH population of 207 lines that were seed propagated and used as the source of microspores, which were cultured following a standard protocol. The number of 
microspores and microspore embryogenic potential, defined as the percentage of microspores developing embryos, were recorded. Embryos at the late cotyledonary stage were transferred to solid medium to induce plantlet regeneration and following a cold treatment, the survival, secondary embryogenesis, direct embryo to plant conversion and root regeneration were scored. Experiments were repeated five times and mean values obtained from 81 to 98 lines were used for QTL mapping was performed based on an Illumina Infinium Brassica 60K SNP molecular linkage. Large and significant genetic differences were observed between the genotypes for all traits. Microspore number varied from 58,900 to $148,700 / \mathrm{ml}$ and microspore embryogenic potential ranged from 0 to $3.6 \%$. Embryo survival varied from $26 \%$ to $99 \%$, and direct embryo to plant conversion was found to range from $13 \%$ to $85 \%$. Root regeneration varied from $14 \%$ to $95 \%$, and secondary embryogenesis occurred in $4 \%$ up to $91 \%$ of the embryos. Analysis of variance revealed a predominant effect of the genotype influencing microspore culture response and high broad-sense heritabilities ranging from $66 \%$ for the microspore number to $86 \%$ for microspore embryogenic potential were detected. A linkage map based on 1,414 SNP markers was developed of which 49 $\%$ exhibited distorted segregation clustered over all linkage groups, except for chromosome A04. Together, ten QTL were mapped on linkage groups A01, A02, A05, A10, C04 and C06. A positive correlation between survival of embryos, direct embryo to plant conversion and root regeneration, as well as overlapping QTL confidence intervals indicated the presence of either a 'hotspot' for loci controlling the regeneration of plants resulting from microspore derived embryos or a pleiotropic gene influencing several traits. BLAST-analysis revealed the presence of nineteen candidate genes within the 95 $\%$ confidence interval of QTL. Among those, CLAVATA3/EMBRYO SURROUNDING REGION-RELATED PEPTIDE 1, CLAVATA3/EMBRYO SURROUNDING REGION-RELATED PEPTIDE 25, WUSCHEL RELATED HOMEOBOX 1, WUSCHEL RELATED HOMEOBOX 2, WUSCHEL RELATED HOMEOBOX 5, CUP-SHAPED COTYLEDON 3, SCARECROW and NOAPICAL-MERISTEM stand out, because they control cell fate and are responsible for root and shoot meristem initiation and maintenance, which is of major factor influencing microspore embryogenic potential and further direct embryo to plant conversion.

In a further step, expression profiles of cold-treated and not cold-treated embryos were assessed through MACE (Massive Analysis of cDNA Ends). Microspore culture of genotypes Express 617 and DH4079 was performed following a standard protocol and 
microspore derived embryos at the late cotyledonary stage were cold treated for 10 days at $2{ }^{\circ} \mathrm{C}$, while control embryos were incubated for 10 days at $20^{\circ} \mathrm{C}$. After this, coldtreated and control embryos were cultured together for 10 days at $20{ }^{\circ} \mathrm{C}$ before they were frozen in liquid nitrogen. MACE was performed by GenXPro GmbH, Frankfurt am Main, Germany. MACE revealed 86,557 different transcripts, 2,606 exhibited a differential expression (log2FoldChange>2.3) of which 207 transcripts were up- or down-regulated in both genotypes when embryos were cold treated. Of the 207 transcripts, 187 transcripts were annotated to known gene sequences and the top ten up- and down-regulated cDNA sequences were used for BLAST analysis to identify orthologous genes in the B. napus, B. oleracea, B. rapa and $A$. thaliana databases. Genes involved in plant defense, stress tolerance and cell detoxification were up-regulated (MATE efflux family protein, AP2/ERF transcription factors, Betv1/MLP like protein), as well as genes encoding for carbohydrate and lipid transporters (SWEET12 and SRPBCC ligand-binding domain-containing protein). Conversely, genes encoding proteins related to stress tolerance with an increasing accumulation during embryo development (M17, LEA49 and glycine-rich protein), were down-regulated in cold-treated embryos.

Results of this study revealed a predominant effect of genotypic factors influencing embryogenic potential and direct embryo to plant conversion in the DH4079 x Express 617 population. QTL analysis and MACE are complementary tools that allow the identification of genes controlling microspore culture response. An increased knowledge of the genetic factors involved in microspore culture would facilitate the allele transfer from high-responsive genotypes to less-responsive lines of agronomic interest and its employment in the development of double haploid populations during plant breeding programs. 


\section{Bibliography}

Abraha E, Bechyne M, Klíma M, Vyvadilová M (2008) Analysis of Factors Affecting Embryogenesis in Microspore Cultures of Brassica carinata. Agric Trop rt Subtrop 41:53-60.

Agarwal P, Agarwal P, Custers J, et al (2006) PCIB an Antiauxin Enhances Microspore Embryogenesis in Microspore Culture of Brassica juncea. Plant Cell Tissue Organ Cult 86:201-210. doi: doi: 10.1007/s11240-006-9108-0

Ahmadi B, Alizadeh K, Teixeira da Silva JA (2012) Enhanced regeneration of haploid plantlets from microspores of Brassica napus L. using bleomycin, PCIB, and phytohormones. Plant Cell, Tissue Organ Cult 109:525-533. doi: 10.1007/s11240012-0119-8

Ahmadi B, Shariatpanahi ME, Teixeira da Silva JA (2014) Efficient induction of microspore embryogenesis using abscisic acid, jasmonic acid and salicylic acid in Brassica napus L. Plant Cell, Tissue Organ Cult 116:343-351. doi: 10.1007/s11240013-0408-x

Aida M, Ishida T, Fukaki H, et al (1997) Genes involved in organ separation in Arabidopsis: an analysis of the cup-shaped cotyledon mutant. Plant Cell 9:841-57. doi: $10.1105 /$ tpc.9.6.841

Aida M, Ishida T, Tasaka M (1999) Shoot apical meristem and cotyledon formation during Arabidopsis embryogenesis: interaction among the CUP-SHAPED COTYLEDON and SHOOT MERISTEMLESS genes. Development 126:1563-1570.

Ali M, Mian M, Custers J, Khurram M (2009) Microspore culture and the performance of microspore derived doubled haploid in Brassica juncea (L.). Bangladesh J Agric Res 33:571-578. doi: 10.3329/bjar.v33i4.2290

Amar S, Becker HC, Möllers C (2008) Genetic Variation and Genotype × Environment Interactions of Phytosterol Content in Three Doubled Haploid Populations of Winter Rapeseed. Crop Sci 48:1000. doi: 10.2135/cropsci2007.10.0578

Ardebili S, Mehran E (2011) Effect of 2, 4-D as a Novel Inducer of Embryogenesis in Microspores of Brassica napus L. Czech J Genet Plant Breed 47:114-122.

Babbar S, Agarwal P (2004) Isolated microspore culture of Brassica: an experimental tool for developmental studies and crop improvement. Indian J ... 3:185-202.

Banno H, Ikeda Y, Niu QW, Chua NH (2001) Overexpression of Arabidopsis ESR1 
induces initiation of shoot regeneration. Plant Cell 13:2609-18. doi: 10.1105/tpc.010234

Barrangou R, Doudna JA (2016) Applications of CRISPR technologies in research and beyond. Nat Biotechnol 34:933-941. doi: 10.1038/nbt.3659

Bélanger S, Clermont I, Esteves P, Belzile F (2016) Extent and overlap of segregation distortion regions in 12 barley crosses determined via a Pool-GBS approach. Theor Appl Genet 129:1393-1404. doi: 10.1007/s00122-016-2711-5

Belmonte, Mark F, Ambrose, et al (2006a) Improved development of microsporederived embryo cultures of Brassica napus cv Topaz following changes in glutathione metabolism. Physiol Plant 127:690-700. doi: doi: 10.1111/j.13993054.2006.00707.x

Belmonte M, Elhiti M, Ashihara H, Stasolla C (2011) Brassinolide-improved development of Brassica napus microspore-derived embryos is associated with increased activities of purine and pyrimidine salvage pathways. Planta 233:95107. doi: $10.1007 / \mathrm{s} 00425-010-1287-6$

Belmonte M, Elhiti M, Waldner B, Stasolla C (2010) Depletion of cellular brassinolide decreases embryo production and disrupts the architecture of the apical meristems in Brassica napus microspore-derived embryos. J Exp Bot 61:2779-94. doi: $10.1093 / \mathrm{jxb} /$ erq110

Belmonte MF, Ambrose SJ, Ross ARS, et al (2006b) Improved development of microspore-derived embryo cultures of Brassica napus cv Topaz following changes in glutathione metabolism. Physiol Plant 127:690-700. doi: 10.1111/j.13993054.2006.00707.x

Biddington NL, Sutherland RA, Robinson HT (1988) Silver Nitrate Increases Embryo Production in Anther Culture of Brussels Sprouts. Ann Bot 62:181-185.

Bojahr J, Nhengiwa O, Krezdorn N, et al (2016) Massive analysis of cDNA ends (MACE) reveals a co-segregating candidate gene for LpPg1 stem rust resistance in perennial ryegrass (Lolium perenne). Theor Appl Genet 129:1915-1932. doi: 10.1007/s00122-016-2749-4

Boldt R, Zrenner R (2003) Purine and pyrimidine biosynthesis in higher plants. Physiol Plant 117:297-304. doi: 10.1034/j.1399-3054.2003.00030.x

Boutilier K, Fiers M, Liu C, van der Geest ALHM (2005) Biochemical and Molecular Aspects of Haploid Embryogenesis. In: Palmer D, Keller WA, Kasha KJ (eds) Haploids in Crop Improvement II. Springer Berlin, Heidelberg, pp 73-95

Boutilier K, Offringa R, Sharma V (2002) Ectopic expression of BABY BO0M triggers a conversion from vegetative to embryonic growth. Plant Cell 14:1737-1749. doi: 10.1105/tpc.001941.tissue

Brand U (2000) Dependence of Stem Cell Fate in Arabidopsis on a Feedback Loop Regulated by CLV3 Activity. Science (80- ) 289:617-619. doi:

10.1126/science.289.5479.617

Brandes H (2014) Variation und Vererbung von Glucosinolatgehalt und - muster in 
Grünmasse und Samen von Raps ( Brassica napus L .) und deren Zusammenhang zum Befall mit Rapsstängelschädlingen. Georg-August-Universität Göttingen, Germany

Brunel D, Froger N, Pelletier G (1999) Development of amplified consensus genetic markers (ACGM) in Brassica napus from Arabidopsis thaliana sequences of known biological function. Genome 42:387-402. doi: 10.1139/gen-42-3-387

Candat A, Paszkiewicz G, Neveu M, et al (2014) The Ubiquitous Distribution of Late Embryogenesis Abundant Proteins across Cell Compartments in Arabidopsis Offers Tailored Protection against Abiotic Stress. Plant Cell 26:1-20. doi: 10.1105/tpc.114.127316

Carles CC, Fletcher JC (2003) Shoot apical meristem maintenance: the art of a dynamic balance. Trends Plant Sci 8:394-401. doi: 10.1016/S1360-1385(03)00164-X

Castillo AM, Nielsen NH, Jensen A, Vallés MP (2014) Effects of n-butanol on barley microspore embryogenesis. Plant Cell, Tissue Organ Cult 117:411-418. doi: $10.1007 / \mathrm{s} 11240-014-0451-2$

Cegielska-Taras T, Tykarska T, Szała L, et al (2002) Direct plant development from microspore-derived embryos of winter oilseed rape Brassica napus L. ssp. oleifera (DC.) Metzger. Euphytica 124:341-347. doi: 10.1023/A:1015785717106

Chalhoub B, Denoeud F, Liu S, et al (2014) Early allopolyploid evolution in the postNeolithic Brassica napus oilseed genome. Science (80- ) 345:950-953. doi: 10.1126/science.1253435

Chen L-Q, Lin IW, Qu X-Q, et al (2015) A cascade of sequentially expressed sucrose transporters in the seed coat and endosperm provides nutrition for the Arabidopsis embryo. Plant Cell 27:607-19. doi: 10.1105/tpc.114.134585

Chen L-Q, Qu X-Q, Hou B-H, et al (2012) Sucrose Efflux Mediated by SWEET Proteins as a Key Step for Phloem Transport. Science (80- ) 335:207-211. doi: 10.1126/science.1213351

Chen W, Zhang Y, Liu X, et al (2007) Detection of QTL for six yield-related traits in oilseed rape (Brassica napus) using DH and immortalized $\mathrm{F}(2)$ populations. Theor Appl Genet 115:849-58. doi: 10.1007/s00122-007-0613-2

Chen Y, Müller F, Rieu I, Winter P (2016) Epigenetic events in plant male germ cell heat stress responses. Plant Reprod 29:21-29. doi: 10.1007/s00497-015-0271-5

Chen ZZ, Snyder S, Fan ZG, Loh WH (1994) Efficient Production of Doubled Haploid Plants through Chromosome Doubling of Isolated Microspores in Brassica napus. Plant Breed 113:217-221. doi: 10.1111/j.1439-0523.1994.tb00725.x

Chinnusamy V, Zhu J, Zhu J-K (2007) Cold stress regulation of gene expression in plants. Trends Plant Sci 12:444-451. doi: 10.1016/j.tplants.2007.07.002

Chuong P V., Beversdorf WD (1985) High frequency embryogenesis through isolated microspore culture in Brassica napus L. and B. carinata Braun. Plant Sci 39:219226. doi: 10.1016/0168-9452(85)90178-5 
and bud sampling on microspore culture of Brassica napus. Can J Bot 66:16531657. doi: $10.1139 / \mathrm{b} 88-225$

Chyi Y-S, Hoenecke ME, Sernyk JL (1992) A genetic linkage map of restriction fragment length polymorphism loci for Brassica rapa (syn. campestris). Genome 35:746-757.

Clark SE, Williams RW, Meyerowitz EM (1997) The CLAVATA1Gene Encodes a Putative Receptor Kinase That Controls Shoot and Floral Meristem Size in Arabidopsis. Cell 89:575-585. doi: 10.1016/S0092-8674(00)80239-1

Clarke WE, Higgins EE, Plieske J, et al (2016) A high-density SNP genotyping array for Brassica napus and its ancestral diploid species based on optimised selection of single-locus markers in the allotetraploid genome. Theor Appl Genet. doi: $10.1007 / \mathrm{s} 00122-016-2746-7$

Cloutier S, Cappadocia M, Landry BS (1995) Study of microspore-culture responsiveness in oilseed rape (Brassica napus L.) by comparative mapping of a F2 population and two microspore-derived populations. Theor Appl Genet 91-91:841-847. doi: 10.1007/BF00223890

Coventry J, Kott L, Beversdorf WD (1988) Manual for microspore culture technique for Brassica napus. 35. doi: 10.13140/RG.2.1.4952.8720

Custers JBM, Cordewener JHG, Fiers MA, et al (2001) Androgenesis in Brassica. In: Current Trends in the Embryology of Angiosperms. Springer Netherlands, Dordrecht, pp 451-470

Custers JBM, Cordewener JHG, Nöllen Y, et al (1994) Temperature controls both gametophytic and sporophytic development in microspore cultures of Brassica napus. Plant Cell Rep 13:267-271. doi: 10.1007/BF00233317

De Smet I, Lau S, Mayer U, Jürgens G (2010) Embryogenesis - the humble beginnings of plant life. Plant J 61:959-70. doi: 10.1111/j.1365-313X.2010.04143.x

Delourme R, Falentin C, Huteau V, et al (2006) Genetic control of oil content in oilseed rape (Brassica napus L.). Theor Appl Genet 113:1331-1345. doi: 10.1007/s00122006-0386-z

Dias J., Martins M. (1999) Effect of silver nitrate on anther culture embryo production of different Brassica oleracea morphotypes. Sci Hortic (Amsterdam) 82:299-307. doi: 10.1016/S0304-4238(99)00052-7

Ding G, Wuhan YM, Li X (2012) Segregation distortion analysis of molecular markers in RIL population of brassica napus. J. Agric. Sci. Technol. 14:56-61.

Divi UK, Krishna P (2009) Brassinosteroid: a biotechnological target for enhancing crop yield and stress tolerance. N Biotechnol 26:131-136. doi: 10.1016/j.nbt.2009.07.006

Divi UK, Krishna P (2010) Overexpression of the Brassinosteroid Biosynthetic Gene AtDWF4 in Arabidopsis Seeds Overcomes Abscisic Acid-induced Inhibition of Germination and Increases Cold Tolerance in Transgenic Seedlings. J Plant Growth Regul 29:385-393. doi: 10.1007/s00344-010-9150-3 
Sparks D (ed) Advances in agronomy, 1st edn. Academic Press Inc., San Diego, California, USA., pp 1-65

Drews GN, Bowman JL, Meyerowitz EM (1991) Negative regulation of the Arabidopsis homeotic gene AGAMOUS by the APETALA2 product. Cell 65:991-1002. doi: 10.1016/0092-8674(91)90551-9

Du C, Hu K, Xian S, et al (2016) Dynamic transcriptome analysis reveals AP2 / ERF transcription factors responsible for cold stress in rapeseed (Brassica napus L .) factors responsible for cold stress in rapeseed ( Brassica napus L .). Mol Genet Genomics 291:1053-1067. doi: 10.1007/s00438-015-1161-0

Dubas E, Janowiak F, Krzewska M, et al (2013) Endogenous ABA concentration and cytoplasmic membrane fluidity in microspores of oilseed rape (Brassica napus L.) genotypes differing in responsiveness to androgenesis induction. Plant Cell Rep 32:1465-1475. doi: 10.1007/s00299-013-1458-6

Dubas E, Moravčíková J, Libantová J, et al (2014) The influence of heat stress on auxin distribution in transgenic B. napus microspores and microspore-derived embryos. Protoplasma 251:1077-1087. doi: 10.1007/s00709-014-0616-1

Dunwell JM, Cornisch M, De Courcel AGL, Middlefell-Williams JE (1983) Induction and Growth of "Microspore-Derived" Embryos of Brassica napus ssp. oleifera. J Exp Bot 34:1768-1778. doi: 10.1093/jxb/34.12.1768

Dunwell JM, Cornish M (1985) Influence of Preculture Variables on Microspore Embryo Production in Brassica napus ssp. oleifera cv. Duplo. Ann Bot 56:281-289.

Echávarri B, Cistué L (2016) Enhancement in androgenesis efficiency in barley (Hordeum vulgare L.) and bread wheat (Triticum aestivum L.) by the addition of dimethyl sulfoxide to the mannitol pretreatment medium. Plant Cell Tissue Organ Cult 125:11-22. doi: 10.1007/s11240-015-0923-z

Ecke W, Kampouridis A, Ziese-Kubon K, Hirsch A-C (2015) Identification and genetic characterization by high-throughput SNP analysis of intervarietal substitution lines of rapeseed (Brassica napus L.) with enhanced embryogenic potential. Theor Appl Genet 128:587-603. doi: 10.1007/s00122-015-2455-7

Elhiti M, Tahir M, Gulden R, et al (2010) Modulation of embryo-forming capacity in culture through the expression of Brassica genes involved in the regulation of the shoot apical meristem. J Exp Bot 61:4069-4085. doi: 10.1093/jxb/erq222

Elhiti M, Wally OSD, Belmonte MF, et al (2013) Gene expression analysis in microdissected shoot meristems of Brassica napus microspore-derived embryos with altered SHOOTMERISTEMLESS levels. Planta 237:1065-1082. doi: $10.1007 / \mathrm{s} 00425-012-1814-8$

Elhiti M, Yang C, Belmonte M, et al (2012) Transcriptional changes of antioxidant responses, hormone signalling and developmental processes evoked by the Brassica napus SHOOTMERISTEMLESS during in vitro embryogenesis. Plant Physiol Biochem. doi: doi: 10.1016/j.plaphy.2012.06.024 
Fábián A, Földesiné Füredi PK, Ambrus H, et al (2015) Effect of n-butanol and cold pretreatment on the cytoskeleton and the ultrastructure of maize microspores when cultured in vitro. Plant Cell, Tissue Organ Cult 123:257-271. doi: 10.1007/s11240-015-0829-9

Fan Z, Armstrong KC, Keller WA (1988) Development of microspores in vivo and in vitro in Brassica napus L. Protoplasma 147:191-199. doi: 10.1007/BF01403347

Fei H, Tsang E, Cutler A (2007) Gene expression during seed maturation in Brassica napus in relation to the induction of secondary dormancy. Genomics 89:419-428. doi: doi: 10.1016/j.ygeno.2006.11.008

Ferrie A (2003) Microspore culture of Brassica species. In: Maluszynski M, Kasha KJ, Forster BP, Szarejko I (eds) Doubled Haploid Production in Crop Plants: A Manual. Springer Netherlands, Dordrecht, pp 205-215

Ferrie A, Dirpaul J, Krishna P, et al (2005) Effects of brassinosteroids on microspore embryogenesis in Brassica species. Vitr Cell Dev Biol - Plant 41:742-745. doi: 10.1079/IVP2005690

Ferrie A, Keller W (2007) Optimization of methods for using polyethylene glycol as a non-permeating osmoticum for the induction of microspore embryogenesis in the Brassicaceae. Vitr Cell Dev Biol 43:348-355. doi: 10.1007/s11627-007-9053-6

Ferrie A, Möllers C (2011) Haploids and doubled haploids in Brassica spp. for genetic and genomic research. Plant Cell, Tissue Organ Cult 104:375-386. doi: 10.1007/s11240-010-9831-4

Foisset N, Delourme R (1996) Segregation distortion in androgenic plants. pp 189-201

Foisset N, Delourme R, Barret P, et al (1996) Molecular-mapping analysis in Brassica napus using isozyme, RAPD and RFLP markers on a doubled-haploid progeny. Theor Appl Genet 93:1017-1025. doi: 10.1007/BF00230119

Foisset N, Delourme R, Lugas MO, Renard M (1993) Segregation Analysis of Isozyme Markers on Isolated Microspore-Derived Embryos in Brassica napus L. Plant Breed 110:315-322. doi: 10.1111/j.1439-0523.1993.tb00595.x

Földesiné Füredi P, Ambrus H, Barnabás B (2012) Development of cultured microspores of maize in the presence of $\mathrm{n}$-butanol and 2-aminoethanol. Acta Agron Hungarica 60:183-189. doi: 10.1556/AAgr.60.2012.3.1

Földesiné Füredi PK, Ambrus H, Barnabás B (2011) The effect of n-butanol and 2aminoethanol on the in vitro androgenesis of maize. Acta Biol Szeged 55:77-78.

Fourmann M, Barret P, Froger N, et al (2002) From Arabidopsis thaliana to Brassica napus: development of amplified consensus genetic markers (ACGM) for construction of a gene map. TAG Theor Appl Genet 105:1196-1206. doi: $10.1007 / \mathrm{s} 00122-002-1040-\mathrm{z}$

Freudenthal JA (2016) Microspore embryogenic potential of the Brassica napus genotypes "Express", "DH4079" and their F1 and comparison of marker segregation in a derived $\mathrm{DH}$ - and $\mathrm{BC}_{1}$ - population. Georg-August-Universität Göttingen, Germany 
Fujimoto SY (2000) Arabidopsis Ethylene-Responsive Element Binding Factors Act as Transcriptional Activators or Repressors of GCC Box-Mediated Gene Expression. Plant Cell Online 12:393-404. doi: 10.1105/tpc.12.3.393

Galili T (2015) dendextend: an R package for visualizing, adjusting and comparing trees of hierarchical clustering. Bioinformatics 31:3718-3720. doi: 10.1093/bioinformatics/btv428

Gallardo K, Job C, Groot SP, et al (2001) Proteomic analysis of arabidopsis seed germination and priming. Plant Physiol 126:835-48. doi: 10.1104/pp.126.2.835

Gamborg OL, Miller R a., Ojima K (1968) Nutrient requirements of suspension cultures of soybean root cells. Exp Cell Res 50:151-158. doi: 10.1016/00144827(68)90403-5

Ge F, Hu Y, Wang J (2013) Spatial and temporal gene expression during seed germination of Brassica napus. Acta Physiol Plant 35:2939-2950. doi: 10.1007/s11738-013-1324-8

George E, Hall M, Klerk G (2008) The Components of Plant Tissue Culture Media ll: Organic Additions, Osmotic and pH Effects, and Support Systems. Plant Propag by tissue Cult 115-173.

Gervais C, Newcomb W, Simmonds DH (2000) Rearrangement of the actin filament and microtubule cytoskeleton during induction of microspore embryogenesis in Brassica napus L. cv. Topas. Protoplasma 213:194-202. doi: 10.1007/BF01282157

Giavalisco P, Nordhoff E, Kreitler T, et al (2005) Proteome analysis of Arabidopsis thaliana by two-dimensional gel electrophoresis and matrix-assisted laser desorption/ionisation-time of flight mass spectrometry. Proteomics 5:1902-1913. doi: $10.1002 /$ pmic.200401062

Gland A, Lichter R, Schweiger H-G (1988) Genetic and Exogenous Factors Affecting Embryogenesis in Isolated Microspore Cultures of Brassica napus L. J Plant Physiol 132:613-617. doi: 10.1016/S0176-1617(88)80264-5

Gu HH, Hagberg P, Zhou WJ (2004) Cold pretreatment enhances microspore embryogenesis in oilseed rape (Brassica napus L.). Plant Growth Regul 42:137143. doi: 10.1023/B:GROW.0000017488.29181.fa

Gu HH, Zhou WJ, Hagberg P (2003) High frequency spontaneous production of doubled haploid plants in microspore cultures of Brassica rapa ssp. chinensis. Euphytica 134:239-245. doi: 10.1023/B:EUPH.0000004945.01455.6d

Gupta SK (ed) (2013) Biotechnology of Crucifers. Springer New York, New York, NY

Haddadi P, Moieni A, Karimzadeh G, Abdollahi MR (2008) Effects of gibberellin, abscisic acid and embryo desiccation on normal plantlet regeneration, secondary embryogenesis and callogenesis in microspore culture of Brassica napus L. cv. PF704. Int J Plant Prod 2:153-162.

Haecker A (2004) Expression dynamics of WOX genes mark cell fate decisions during early embryonic patterning in Arabidopsis thaliana. Development 131:657-668. doi: 10.1242/dev.00963 
Hansen M (2000) ABA Treatment and Desiccation of Microspore-derived Embryos of Cabbage (Brassica oleracea ssp. capitata L.) Improves Plant Development. J Plant Physiol 156:164-167. doi: 10.1016/S0176-1617(00)80301-6

Hansen M, Svinnset K (1993) Microspore culture of swede (Brassica napus ssp. rapifera) and the effects of fresh and conditioned media. Plant Cell Rep 12:496500. doi: 10.1007/BF00236094

Häntzschel KR, Weber G (2010) Blockage of mitosis in maize root tips using colchicinealternatives. Protoplasma 241:99-104. doi: 10.1007/s00709-009-0103-2

Hays D, Mandel R, Pharis R (2001) Hormones in zygotic and microspore embryos of Brassica napus. Plant Growth Regul 35:47-58. doi: 10.1023/A:1013831116996

Heberle H, Meirelles GV, da Silva FR, et al (2015) InteractiVenn: a web-based tool for the analysis of sets through Venn diagrams. BMC Bioinformatics 16:169. doi: 10.1186/s12859-015-0611-3

Helariutta Y, Fukaki H, Wysocka-Diller J, et al (2000) The SHORT-ROOT Gene Controls Radial Patterning of the Arabidopsis Root through Radial Signaling. Cell 101:555567. doi: 10.1016/S0092-8674(00)80865-X

Hibara K -i., Karim MR, Takada S, et al (2006) Arabidopsis CUP-SHAPED COTYLEDON3 Regulates Postembryonic Shoot Meristem and Organ Boundary Formation. PLANT CELL ONLINE 18:2946-2957. doi: 10.1105/tpc.106.045716

Hill J, Becker HC, Tigerstedt PMA (1998) Quantitative and Ecological Aspects of Plant Breeding. Springer Netherlands, Dordrecht

Holdsworth MJ, Bentsink L, Soppe WJJ (2008) Molecular networks regulating Arabidopsis seed maturation, after-ripening, dormancy and germination. New Phytol 179:33-54. doi: 10.1111/j.1469-8137.2008.02437.x

Holme IB, Torp AM, Hansen LN, Andersen SB (2004) Quantitative trait loci affecting plant regeneration from protoplasts of Brassica oleracea. Theor Appl Genet 108:1513-1520. doi: 10.1007/s00122-003-1570-z

Hoseini M, Ghadimzadeh M, Ahmadi B, Teixeira da Silva JA (2014) Effects of ascorbic acid, alpha-tocopherol, and glutathione on microspore embryogenesis in Brassica napus L. Vitr Cell Dev Biol - Plant 50:26-35. doi: 10.1007/s11627-013-9579-8

Hosp J, de Maraschin SF, Touraev A, Boutilier K (2007) Functional genomics of microspore embryogenesis. Euphytica 158:275-285. doi: 10.1007/s10681-0069238-9

Huang B, Bird S, Kemble R, et al (1990) Effects of culture density, conditioned medium and feeder cultures on microspore embryogenesis in Brassica napus L. cv. Topas. Plant Cell Rep 8:594-597. doi: 10.1007/BF00270061

Hyodo H, Yamakawa S, Takeda Y, et al (2003) Active gene expression of a xyloglucan endotransglucosylase/hydrolase gene, XTH9, in inflorescence apices is related to cell elongation in Arabidopsis thaliana. Plant Mol Biol 52:473-482. doi: 10.1023/A:1023904217641 
Ilić-Grubor K, Attree SM, Fowke LC (1998) Induction of microspore-derived embryos of Brassica napus L. with polyethylene glycol (PEG) as osmoticum in a low sucrose medium. Plant Cell Rep 17:329-333. doi: 10.1007/s002990050402

Iqbal M, Möllers C, Robbelen G (1994) Increased embryogenesis after colchicine treatment of microspore cultures of Brassica napus L. J Plant Physiol 143:222-226. doi: 10.1016/S0176-1617(11)81691-3

Islam SMS, Tuteja N (2012) Enhancement of androgenesis by abiotic stress and other pretreatments in major crop species. Plant Sci 182:134-144. doi: 10.1016/j.plantsci.2011.10.001

Jacquard C, Nolin F, Hécart C, et al (2009) Microspore embryogenesis and programmed cell death in barley: effects of copper on albinism in recalcitrant cultivars. Plant Cell Rep 28:1329-1339. doi: 10.1007/s00299-009-0733-z

Jenik PD, Gillmor CS, Lukowitz W (2007) Embryonic Patterning in Arabidopsis thaliana. Annu Rev Cell Dev Biol 23:207-236. doi:

10.1146/annurev.cellbio.22.011105.102609

Jett LW, Welbaum GE, Morse RD (1996) Effects of Matric and Osmotic Priming Treatments on Broccoli Seed Germination. J Amer Soc Hort Sci 121:423-429.

Jiang W, Yao Q, Zhang X, et al (2008) Influence of activated charcoal and agitation culture on frequency of embryogenesis from microspore of Chinese cabbage. China Cucurbits Veg 1-3.

Joosen R, Cordewener J, Supena EDJ, et al (2007) Combined Transcriptome and Proteome Analysis Identifies Pathways and Markers Associated with the Establishment of Rapeseed Microspore-Derived Embryo Development. PLANT Physiol 144:155-172. doi: 10.1104/pp.107.098723

Kabir KMR, Kwon S, Park YJ (2013) Application of Cobalt Chloride and Silver Nitrate for Efficient Microspore Culture of Brassica rapa ssp. Plant Tissue Cult Biotechnol 23:1-10. doi: 10.3329/ptcb.v23i1.15554

Kampouridis A, Ziese-Kubon K, Nurhasanah, Ecke W (2016a) Identification and evaluation of intervarietal substitution lines of rapeseed (Brassica napus L.) with donor segments affecting the direct embryo to plant conversion rate of microsporederived embryos. Euphytica 209:181-198. doi: 10.1007/s10681-016-1732-0

Kampouridis A, Ziese-Kubon K, Nurhasanah, Ecke W (2016b) Identification and evaluation of intervarietal substitution lines of rapeseed (Brassica napus L.) with donor segments affecting the diploidization rate of isolated microspores. Euphytica 209:181-198. doi: 10.1007/s10681-016-1656-8

Kinoshita A, Nakamura Y, Sasaki E, et al (2007) Gain-of-Function Phenotypes of Chemically Synthetic CLAVATA3/ESR-Related (CLE) Peptides in Arabidopsis thaliana and Oryza sativa. Plant Cell Physiol 48:1821-1825. doi: $10.1093 / \mathrm{pcp} / \mathrm{pcm} 154$

Kitashiba H, Taguchi K, Kaneko I, et al (2016) Identification of loci associated with embryo yield in microspore culture of Brassica rapa by segregation distortion analysis. Plant Cell Rep 35:2197-2204. doi: 10.1007/s00299-016-2029-4 
Klutschewski S (2013) Methodical improvements in microspore culture of Brassica napus L. Georg-August-Universität Göttingen, Germany.

https://ediss.uni-goettingen.de/handle/11858/00-1735-0000-0001-BC20-2

Kong D, Ju C, Parihar A, et al (2015) Arabidopsis glutamate receptor homolog3.5 modulates cytosolic Ca2+ level to counteract effect of abscisic acid in seed germination. Plant Physiol 167:1630-42. doi: 10.1104/pp.114.251298

Kontowski S, Friedt W (1994) Genotypic effects on microspore culture in a breeding program for high erucic acid content of Brassica napus. Bull. GCIRC 10:30-38.

Kott LS, Beversdorf WD (1990) Enhanced plant regeneration from microspore-derived embryos of Brassica napus by chilling, partial desiccation and age selection. Plant Cell Tissue Organ Cult 23:187-192. doi: 10.1007/BF00034430

Kott LS, Polsoni L, Beversdorf WD (1988a) Cytological aspects of isolated microspore culture of Brassica napus. Can J Bot 66:1658-1664. doi: 10.1139/b88-226

Kott LS, Polsoni L, Ellis B, Beversdorf WD (1988b) Autotoxicity in isolated microspore cultures of Brassica napus. Can J Bot 66:1665-1670. doi: 10.1139/b88-227

Krishna P (2003) Brassinosteroid-Mediated Stress Responses. J Plant Growth Regul 22:289-297. doi: 10.1007/s00344-003-0058-z

Kubala S, Garnczarska M, Wojtyla Ł, et al (2015) Deciphering priming-induced improvement of rapeseed (Brassica napus L.) germination through an integrated transcriptomic and proteomic approach. Plant Sci 231:94-113. doi:

10.1016/j.plantsci.2014.11.008

Laux T, Mayer KF, Berger J, Jurgens G (1996) The WUSCHEL gene is required for shoot and floral meristem integrity in Arabidopsis. Development 122:87-96.

Leroux B, Carmoy N, Giraudet D, et al (2009) Inhibition of ethylene biosynthesis enhances embryogenesis of cultured microspores of Brassica napus. Plant Biotechnol Rep 3:347-353. doi: 10.1007/s11816-009-0109-4

Li F, Wu X, Tsang E, Cutler AJ (2005) Transcriptional profiling of imbibed Brassica napus seed. Genomics 86:718-730. doi: 10.1016/j.ygeno.2005.07.006

Li Y, Qiu L, Huang L, Cao J (2012) Molecular Cloning and Characterization of BcMF22, a Novel Gene Related to Pollen Development and Fertilization in Brassica campestris ssp. chinensis. Plant Mol Biol Report 30:860-866. doi: 10.1007/s11105-011-03933

Lichter R (1989) Efficient Yield of Embryoids by Culture of Isolated Microspores of Different Brassicaceae Species. Plant Breed 103:119-123. doi: 10.1111/j.14390523.1989.tb00359.x

Lichter R (1982) Induction of Haploid Plants From Isolated Pollen of Brassica napus. Zeitschrift für Pflanzenphysiologie 105:427-434. doi: 10.1016/S0044328X(82)80040-8

Lichter R (1985) From microspores to rape plants: A tentative way to low glucosinolate strains. In: Sorensen H (ed) Advances in the production and utilization of cruciferous crops. Springer Netherlands, pp 268-277 
Lichter R, Groot E, Fiebig D (1988) Glucosinolates Determined by HPLC in the Seeds of Microspore ?Derived Homozygous Lines of Rapeseed (Brassica napus L.). Plant Breed 221:209-222.

Liu D, Li W, Cheng J, Hou L (2015) AtPGK2, a member of PGKs gene family in Arabidopsis, has a positive role in salt stress tolerance. Plant Cell, Tissue Organ Cult 120:251-262. doi: 10.1007/s11240-014-0601-6

Liu S, Liu Y, Yang X, et al (2014) The Brassica oleracea genome reveals the asymmetrical evolution of polyploid genomes. Nat Commun 5:3930. doi: 10.1038/ncomms4930

Liu T-W, Wu F-H, Wang W-H, et al (2011) Effects of calcium on seed germination, seedling growth and photosynthesis of six forest tree species under simulated acid rain. Tree Physiol 31:402-413. doi: 10.1093/treephys/tpr019

Lo S-F, Yang S-Y, Chen K-T, et al (2008) A Novel Class of Gibberellin 2-Oxidases Control Semidwarfism, Tillering, and Root Development in Rice. PLANT CELL ONLINE 20:2603-2618. doi: 10.1105/tpc.108.060913

Lombard V, Delourme R (2001) A consensus linkage map for rapeseed (Brassica napus L.): construction and integration of three individual maps from $\mathrm{DH}$ populations. TAG Theor Appl Genet 103:491-507. doi: 10.1007/s001220100560

Lv Y, Fu S, Chen S, et al (2016) Ethylene response factor BnERF2-like (ERF2.4) from Brassica napus L. enhances submergence tolerance and alleviates oxidative damage caused by submergence in Arabidopsis thaliana. Crop J 4:1-13. doi: 10.1016/j.cj.2016.01.004

Mackay T, Stone E, Ayroles J (2009) The genetics of quantitative traits: challenges and prospects. Nat Rev Genet 10:565-577. doi: 10.1038/nrg2612

Malik MR, Krochko JE (2009) Gene Expression Profiling of Microspore Embryogenesis in Brassica napus. In: Touraev A, Forster BP, Jain SM (eds) Advances in Haploid Production in Higher Plants. Springer Netherlands, Dordrecht, pp 115-125

Malik MR, Rangaswamy NS, Shivanna KR (2001) Induction of microspore embryos in a CMS line of Brassica juncea and formation of the androgenic plantlets. Euphytica 120:195-203. doi: 10.1023/A:1017502113066

Malik MR, Wang F, Dirpaul JM, et al (2007) Transcript profiling and identification of molecular markers for early microspore embryogenesis in Brassica napus. Plant Physiol 144:134-54. doi: 10.1104/pp.106.092932

Malik MR, Wang F, Dirpaul JM, et al (2008) Isolation of an embryogenic line from nonembryogenic Brassica napus cv. Westar through microspore embryogenesis. J Exp Bot 59:2857-73. doi: 10.1093/jxb/ern149

Maraschin SF (2005) Androgenic switch: an example of plant embryogenesis from the male gametophyte perspective. J Exp Bot 56:1711-1726. doi: 10.1093/jxb/eri190

Mathias R (1988) An Improved In Vitro Culture Procedure for Embryoids Derived from Isolated Microspores of Rape (Brassica napus L.). Plant Breed 100:320-322. doi: 10.1111/j.1439-0523.1988.tb00259.x 
Matsuo N, Mase H, Makino M, et al (2009) Identification of ENHANCER OF SHOOT REGENERATION 1-upregulated genes during in vitro shoot regeneration. Plant Biotechnol 26:385-393. doi: 10.5511/plantbiotechnology.26.385

Meinke DW, Franzmann LH, Nickle TC, Yeung EC (1994) Leafy Cotyledon Mutants of Arabidopsis. 6:1049-1064.

Mitchell A, Chang H-Y, Daugherty L, et al (2015) The InterPro protein families database: the classification resource after 15 years. Nucleic Acids Res 43:D213-D221. doi: $10.1093 / \mathrm{nar} / \mathrm{gku} 1243$

Möllers C, Iqbal M, Röbbelen G (1994) Efficient production of doubled haploid Brassica napus plants by colchicine treatment of microspores. Euphytica 95-104.

Möllers C, Iqbal MCM (2009) Doubled Haploids in Breeding Winter Oilseed Rape. In: Touraev A, Forster B, Jain S (eds) Advances in Haploid Production in Higher Plants. Springer Netherlands, Dordrecht, pp 161-169

Murashige T, Skoog F (1962) a Revised Medium for Rapid Growth and Bio Assays With Tobacco Tissue Cultures. Physiol Plant 15:473-497. doi: 10.1111/j.13993054.1962.tb08052.x

Na H, Kwak J-H, Chun C (2011) The effects of plant growth regulators, activated charcoal, and AgNO3 on microspore derived embryo formation in broccoli (Brassica oleracea L. var. italica). Hortic Environ Biotechnol 52:524-529. doi: 10.1007/s13580-011-0034-7

Nakabayashi K, Okamoto M, Koshiba T, et al (2005) Genome-wide profiling of stored mRNA in Arabidopsis thaliana seed germination: Epigenetic and genetic regulation of transcription in seed. Plant J 41:697-709. doi: 10.1111/j.1365313X.2005.02337.x

Ni J, Clark SE (2006) Evidence for Functional Conservation, Sufficiency, and Proteolytic Processing of the CLAVATA3. Plant Physiol 140:726-733. doi: 10.1104/pp.105.072678.heterodimer

Nieuwenhuis R, Grotenhuis M te, Pelzer B (2012) Influence.ME: Tools for Detecting Influential Data in Mixed Effects Models. R J 4:38-47.

Nitsch C, Nitsch JP (1967) The induction of flowering in vitro in stem segments of Plumbago indica L. Planta 72:355-370. doi: 10.1007/BF00390146

Nuutila A., Hämäläinen J, Mannonen L (2000) Optimization of media nitrogen and copper concentrations for regeneration of green plants from polyembryogenic cultures of barley (Hordeum vulgare L.). Plant Sci 151:85-92. doi: 10.1016/S01689452(99)00202-2

Orr W, Johnson-Flanagan AM, Keller WA, Singh J (1990) Induction of freezing tolerance in microspore-derived embryos of winter Brassica napus. Plant Cell Rep 8:579581. doi: 10.1007/BF00270057

Pace R, Benincasa P, Ghanem ME, et al (2012) Germination of Untreated and Primed Seeds in Rapeseed (Brassica Napus Var Oleifera Del.) Under Salinity and Low Matric Potential. Exp Agric 48:238-251. doi: 10.1017/S0014479711001189 
Parcy F, Valon C, Raynal M, et al (1994) Regulation of Gene Expression Programs during Arabidopsis Seed Development : Roles of the AB13 Locus and of Endogenous Abscisic Acid. 6:1567-1582.

Pechan PM, Keller W a (1988) Identification of potentially embryogenic microspores in Brassica napus. Physiol Plant 74:377-384. doi: 10.1111/j.13993054.1988.tb00646.x

Pechan PM, Keller WA (1989) Induction of microspore embryogenesis in Brassica napus L. by gamma irradiation and ethanol stress. Vitr Cell Dev Biol 25:1073-1074. doi: 10.1007/BF02624144

Prem D, Gupta K, Agnihotri A (2005) Effect of various exogenous and endogenous factors on microspore embryogenesis in Indian mustard (Brassica juncea (L.) Czern and Coss). Vitr Cell Dev Biol - Plant 41:266-273. doi: 10.1079/IVP2005636

Prem D, Gupta K, Sarkar G, Agnihotri A (2008) Activated charcoal induced high frequency microspore embryogenesis and efficient doubled haploid production in Brassica juncea. Plant Cell Tissue Organ Cult 93:269-282. doi: 10.1007/s11240008-9373-1

Qiu D, Morgan C, Shi J, et al (2006) A comparative linkage map of oilseed rape and its use for QTL analysis of seed oil and erucic acid content. Theor Appl Genet 114:6780. doi: $10.1007 / \mathrm{s} 00122-006-0411-2$

R Development Core Team (2016) R: A language and environment for statistical computing.

Radoev M (2007) Genetic Analysis of Heterosis in Rapeseed ( B . napus L .) by QTL Mapping. Georg-August-Universität Göttingen, Germany

Radoev M, Becker H, Ecke W (2008) Genetic analysis of heterosis for yield and yield components in rapeseed (Brassica napus L.) by quantitative trait locus mapping. Genetics 179:1547-58. doi: 10.1534/genetics.108.089680

Rahman M, Michalak de Jiménez M (2016) Behind the scenes of microspore-based double haploid development in Brassica napus: A review. J Plant Sci Mol Breed 5:1. doi: 10.7243/2050-2389-5-1

Ramesar-Fortner NS, Yeung EC (2006) Physiological influences in the development and function of the shoot apical meristem of microspore-derived embryos of Brassica napus "Topas." Can J Bot 84:371-383. doi: 10.1139/b06-007

Raynal M, Guilleminot J, Gueguen C, et al (1999) Structure, organization and expression of two closely related novel Lea (late-embryogenesis-abundant) genes in Arabidopsis thaliana. Plant Mol Biol 40:153-165. doi: 10.1023/A:1026403215270

Rieseberg LH, Archer MA, Wayne RK (1999) Transgressive segregation, adaptation and speciation. Heredity (Edinb) 83 ( Pt 4):363-372. doi: 10.1038/sj.hdy.6886170

Rieu I, Eriksson S, Powers SJ, et al (2008a) Genetic analysis reveals that C19-GA 2oxidation is a major gibberellin inactivation pathway in Arabidopsis. Plant Cell 20:2420-36. doi: 10.1105/tpc.108.058818 
Rieu I, Ruiz-Rivero 0, Fernandez-Garcia N, et al (2008b) The gibberellin biosynthetic genes AtGA20ox1 and AtGA20ox2 act, partially redundantly, to promote growth and development throughout the Arabidopsis life cycle. Plant J 53:488-504. doi: 10.1111/j.1365-313X.2007.03356.x

Sabatini S (2003) SCARECROW is involved in positioning the stem cell niche in the Arabidopsis root meristem. Genes Dev 17:354-358. doi: 10.1101/gad.252503

Saleh A, Pages M (2003) Plant AP2/ERF transcription factors. Genetika 18-26.

Sarkar AK, Luijten M, Miyashima S, et al (2007) Conserved factors regulate signalling in Arabidopsis thaliana shoot and root stem cell organizers. Nature 446:811-814. doi: 10.1038/nature05703

Schmid M, Davison TS, Henz SR, et al (2005) A gene expression map of Arabidopsis thaliana development. Nat Genet 37:501-6. doi: 10.1038/ng1543

Schoof H, Lenhard M, Haecker A, et al (2000) The Stem Cell Population of Arabidopsis Shoot Meristems Is Maintained by a Regulatory Loop between the CLAVATA and WUSCHEL Genes. Cell 100:635-644. doi: 10.1016/S0092-8674(00)80700-X

Senaratna T, Kott L, Beversdorf W, McKersie B (1991) Desiccation of microspore derived embryos of oilseed rape (Brassica napus L.). Plant Cell Rep 10:342-344. doi: 10.1007/BF00193155

Seo MS, Jin M, Lee SS, et al (2013) Mapping quantitative trait loci for tissue culture response in VCS3M-DH population of Brassica rapa. Plant Cell Rep 32:1251-1261. doi: $10.1007 / \mathrm{s} 00299-013-1433-2$

Shaikh F, Gul B, Li W, et al (2007) Effect of calcium and light on the germination of Urochondra setulosa under different salts. J Zhejiang Univ Sci B 8:20-26. doi: 10.1631/jzus.2007.B0020

Siebel J, Pauls KP (1989) A comparison of anther and microspore culture as a breeding tool in Brassica napus. Theor Appl Genet 78:473-479. doi: 10.1007/BF00290830

Simmonds DH, Keller WA (1999) Significance of preprophase bands of microtubules in the induction of microspore embryogenesis of Brassica napus. Planta 208:383-391. doi: $10.1007 /$ s004250050573

Smýkalová I, Větrovcová M, Klíma M, et al (2006) Efficiency of Microspore Culture for Doubled Haploid Production in the Breeding Project “Czech Winter Rape." Czech J Genet Plant Breed 2006:58-71.

Soeda Y, Konings MCJM, Vorst O, et al (2005) Gene expression programs during Brassica oleracea seed maturation, osmopriming, and germination are indicators of progression of the germination process and the stress tolerance level. Plant Physiol 137:354-368. doi: 10.1104/pp.104.051664.mobilization

Solis M-T, Rodriguez-Serrano M, Meijon M, et al (2012) DNA methylation dynamics and MET1a-like gene expression changes during stress-induced pollen reprogramming to embryogenesis. J Exp Bot 63:6431-6444. doi: 10.1093/jxb/ers298

Soriano M, Cistué L, Castillo a M (2008) Enhanced induction of microspore embryogenesis after n-butanol treatment in wheat (Triticum aestivum L.) anther culture. Plant Cell Rep 27:805-11. doi: 10.1007/s00299-007-0500-y 
Soriano M, Li H, Boutilier K (2013) Microspore embryogenesis: establishment of embryo identity and pattern in culture. Plant Reprod 26:181-196. doi: 10.1007/s00497-013-0226-7

Sotiropoulos TE, Dimassi KN (2004) Response to increasing rates of boron and $\mathrm{NaCl}$ on shoot proliferation and chemical composition of in vitro kiwifruit shoot cultures. Plant Cell Tissue Organ Cult 79:285-289. doi: 10.1007/s11240-004-4609-1

Souer E, Van Houwelingen A, Kloos D, et al (1996) The no apical Meristem gene of petunia is required for pattern formation in embryos and flowers and is expressed at meristem and primordia boundaries. Cell 85:159-170. doi: 10.1016/S00928674(00)81093-4

Sparrow P a C, Townsend TM, Morgan CL, et al (2004) Genetic analysis of in vitro shoot regeneration from cotyledonary petioles of Brassica oleracea. Theor Appl Genet 108:1249-1255. doi: 10.1007/s00122-003-1539-y

Stam P (1993) Construction of integrated genetic linkage maps by means of a new computer package: Join Map. Plant J 3:739-744. doi: 10.1111/j.1365313X.1993.00739.x

Stasolla C (2010) Glutathione redox regulation of in vitro embryogenesis. Plant Physiol Biochem 48:319-327. doi: 10.1016/j.plaphy.2009.10.007

Stasolla C, Belmonte MF, Tahir M, et al (2008) Buthionine sulfoximine (BSO)-mediated improvement in cultured embryo quality in vitro entails changes in ascorbate metabolism, meristem development and embryo maturation. Planta 228:255-72. doi: $10.1007 / \mathrm{s} 00425-008-0735-\mathrm{z}$

Stasolla C, Katahira R, Thorpe T a, Ashihara H (2003) Purine and pyrimidine nucleotide metabolism in higher plants. J Plant Physiol 160:1271-1295. doi: 10.1078/01761617-01169

Stasolla C, Loukanina N, Ashihara H, et al (2001) Ascorbic acid changes the pattern of purine metabolism during germination of white spruce somatic embryos. 359-367.

Stasolla C, Yeung EC (2006) Endogenous ascorbic acid modulates meristem reactivation in white spruce somatic embryos and affects thymidine and uridine metabolism. Tree Physiol 26:1197-1206. doi: 10.1093/treephys/26.9.1197

Stasolla C, Yeung EC (2007) Cellular ascorbic acid regulates the activity of major peroxidases in the apical poles of germinating white spruce (Picea glauca) somatic embryos. Plant Physiol Biochem 45:188-198. doi: 10.1016/j.plaphy.2007.02.007

Steward CN (2008) Plant Biotechnology and Genetics. John Wiley \& Sons, Inc., Hoboken, NJ, USA

Su Y, Liu Y, Li Z, et al (2015) QTL Analysis of Head Splitting Resistance in Cabbage (Brassica oleracea L. var. capitata) Using SSR and InDel Makers Based on WholeGenome Re-Sequencing. PLoS One 10:1-17. doi: 10.1371/journal.pone.0138073

Supena E, Suharsono S, Jacobsen E, Custers J (2006) Successful development of a shedmicrospore culture protocol for doubled haploid production in Indonesian hot pepper (Capsicum annuum L.). Plant Cell Rep 25:1-10. doi: 10.1007/s00299-0050028-y 
Supena E, Winarto B, Riksen T, et al (2008) Regeneration of zygotic-like microsporederived embryos suggests an important role for the suspensor in early embryo patterning. J Exp Bot 59:803-814. doi: 10.1093/jxb/erm358

Suprianto E (2014) Genetic variation and inheritance of seed fibre content in winter oilseed rape (Brassica napus L.). Georg-August-Universität Göttingen, Germany

TAIR (2016) The Arabidopsis Information Resource. www.arabidopsis.org. Accessed 16 Aug 2016

Takada S, Hibara K, Ishida T, Tasaka M (2001) The CUP-SHAPED COTYLEDON1 gene of Arabidopsis regulates shoot apical meristem formation. Development 128:112735.

Takahashi Y, Yokoi S, Takahata Y (2012) Effects of genotypes and culture conditions on microspore embryogenesis and plant regeneration in several subspecies of Brassica rapa L. Plant Biotechnol Rep 1-8. doi: doi: 10.1007/s11816-012-0224-5

Takahata Y, Brown DCW, Keller W a., Kaizuma N (1993) Dry artificial seeds and desiccation tolerance induction in microspore-derived embryos of broccoli. Plant Cell Tissue Organ Cult 35:121-129. doi: 10.1007/BF00032961

Takahira J, Cousin a., Nelson MN, Cowling W a. (2010) Improvement in efficiency of microspore culture to produce doubled haploid canola (Brassica napus L.) by flow cytometry. Plant Cell, Tissue Organ Cult 104:51-59. doi: 10.1007/s11240-0109803-8

Tanhuanpää PK, Vilkki JP, Vilkki HJ (1994) Segregation and linkage analysis of DNA markers in microspore derived and F2 populations of oilseed rape (Brassica napus L.). Euphytica 74:59-65. doi: 10.1007/BF00033768

Teh L, Möllers C (2016) Genetic variation and inheritance of phytosterol and oil content in a doubled haploid population derived from the winter oilseed rape Sansibar $x$ Oase cross. Theor Appl Genet 129:181-199. doi: 10.1007/s00122-015-2621-y

Telmer CA, Simmonds DH, Newcomb W (1992) Determination of developmental stage to obtain high frequencies of embryogenic microspores in Brassica napus. Physiol Plant 84:417-424. doi: 10.1111/j.1399-3054.1992.tb04685.x

Teutonico RA, Osborn TC (1994) Mapping of RFLP and qualitative trait loci in Brassica rapa and comparison to the linkage maps of B. napus, B. oleracea, and Arabidopsis thaliana. Theor Appl Genet 89-89:885-894. doi: 10.1007/BF00224514

Thomas TD (2008) The role of activated charcoal in plant tissue culture. Biotechnol Adv 26:618-631. doi: 10.1016/j.biotechadv.2008.08.003

Thurling N, Chay PM (1984) The Influence of Donor Plant Genotype and Environment on Production of Multicellular Microspores in Cultured Anthers of Brassica napus ssp. oleifera. Ann Bot 54:681-693.

Tian H, Yao CY, Sun MX (2004) High Frequency Conversion of Microspore-Derived Embryos of Brassica napus cv. Topas by Supplemental Calcium and Vitamins. Plant Cell Tissue Organ Cult 76:159-165. doi: 10.1023/B:TICU.0000007292.10767.54 
Tobe K, Li X, Omasa K (2004) Effects of five different salts on seed germination and seedling growth of Haloxylon ammodendron (Chenopodiaceae). Seed Sci Res 14:345-353. doi: 10.1079/SSR2004188

Touraev A, Pfosser M, Heberle-Bors E (2001) The microspore: A haploid multipurpose cell.

Touraev A, Vicente 0, Heberle-Bors E (1997) Initiation of microspore embryogenesis by stress. Trends Plant Sci 2:297-302. doi: 10.1016/S1360-1385(97)89951-7

Trifonova A, Atanassov A (1997) Microspore Culture of Winter Oil Rapeseed ( Brassica Napus ): II. Embryo Conversion, Plant Regeneration and Double Haploid Production of Brassica Napus L. Biotechnol Biotechnol Equip 11:31-35. doi: 10.1080/13102818.1997.10818949

Tsuwamoto R, Fukuoka H, Takahata Y (2007) Identification and characterization of genes expressed in early embryogenesis from microspores of Brassica napus. Planta 225:641-52. doi: 10.1007/s00425-006-0388-8

Tsuwamoto R, Takahata Y (2008) Identification of genes specifically expressed in androgenesis-derived embryo in rapeseed (Brassica napus L.). Breed Sci 58:251259. doi: $10.1270 /$ jsbbs.58.251

UniProt (2015) UniProt: a hub for protein information. Nucleic Acids Res 43:D204D212. doi: 10.1093/nar/gku989

Utz HF (2011) PLABSTAT-A computer program for statistical analysis of plant breeding experiments 3.A.

Uzunova M, Ecke W, Weissleder K, Röbbelen G (1995) Mapping the genome of rapeseed (Brassica napus L.). I. Construction of an RFLP linkage map and localization of QTLs for seed glucosinolate content. Theor Appl Genet 90:194-204. doi:

10.1007/BF00222202

Viswanathan C, Zhu J-K (2002) Molecular genetic analysis of cold-regulated gene transcription. Philos Trans R Soc B Biol Sci 357:877-886. doi:

$10.1098 /$ rstb.2002.1076

Voorrips RE, Jongerius MC, Kanne HJ (1997) Mapping of two genes for resistance to clubroot (Plasmodiophora brassicae) in a population of doubled haploid lines of Brassica oleracea by means of RFLP and AFLP markers. Theor Appl Genet 94:7582. doi: $10.1007 / \mathrm{s} 001220050384$

Walley PG, Carder J, Skipper E, et al (2012) A new broccoli $\times$ broccoli immortal mapping population and framework genetic map: tools for breeders and complex trait analysis. Theor Appl Genet 124:467-484. doi: 10.1007/s00122-011-1721-6

Wang S, Basten CJ, Zeng Z-B (2012) Windows QTL Cartographer 2.5.

Wang XX, Wang HHH, Wang JJJ, et al (2011) The genome of the mesopolyploid crop species Brassica rapa. Nat Genet 43:1035-1039. doi: 10.1038/ng.919

Wang Z, Gerstein M, Snyder M (2009) RNA-Seq: a revolutionary tool for transcriptomics. Nat Rev Genet 10:57-63. doi: 10.1038/nrg2484 
Weber S, Ünker F, Friedt W (2005) Improved doubled haploid production protocol for Brassica napus using microspore colchicine treatment in vitro and ploidy determination by flow cytometry. Plant Breed 124:511-513.

Wen J, Vanek-Krebitz M, Hoffmann-Sommergruber K, et al (1997) The Potential of Betv1 Homologues, a Nuclear Multigene Family, as Phylogenetic Markers in Flowering Plants. Mol Phylogenet Evol 8:317-333. doi: http://dx.doi.org/10.1006/mpev.1997.0447

White PJ, Broadley MR (2003) Calcium in plants. Ann Bot 92:487-511. doi: $10.1093 / \mathrm{aob} / \mathrm{mcg} 164$

Wickramasuriya AM, Dunwell JM (2015) Global scale transcriptome analysis of Arabidopsis embryogenesis in vitro. BMC Genomics 16:1-23. doi: 10.1186/s12864015-1504-6

Wojnarowiez G, Jacquard C, Devaux P, et al (2002) Influence of copper sulfate on anther culture in barley (Hordeum vulgare L.). Plant Sci 162:843-847. doi: 10.1016/S0168-9452(02)00036-5

Xie Q (2000) Arabidopsis NAC1 transduces auxin signal downstream of TIR1 to promote lateral root development. Genes Dev 14:3024-3036. doi: $10.1101 /$ gad.852200

Yamaguchi S (2008) Gibberellin Metabolism and its Regulation. Annu Rev Plant Biol 59:225-251. doi: 10.1146/annurev.arplant.59.032607.092804

Yamauchi Y, Takeda-Kamiya N, Hanada A, et al (2007) Contribution of Gibberellin Deactivation by AtGA2ox2 to the Suppression of Germination of Dark-Imbibed Arabidopsis thaliana Seeds. Plant Cell Physiol 48:555-561. doi: $10.1093 / \mathrm{pcp} / \mathrm{pcm} 023$

Yang HAN, Xue-ling YE, Hui F, et al (2014) Improved efficiency of microspore culture of Brassica campestris ssp . pekinensis ( Chinese cabbage ). 9:2111-2118. doi: 10.5897/AJAR09.758

Yang J, Hu C, Hu H, et al (2008) QTLNetwork: mapping and visualizing genetic architecture of complex traits in experimental populations. Bioinformatics 24:721723. doi: 10.1093/bioinformatics/btm 494

Yu X, Wang H, Zhong W, et al (2013) QTL Mapping of Leafy Heads by Genome Resequencing in the RIL Population of Brassica rapa. PLoS One 8:e76059. doi: 10.1371/journal.pone.0076059

Yuan S, Su Y, Liu Y, et al (2012) Effects of pH, MES, arabinogalactan-proteins on microspore cultures in white cabbage. Plant Cell, Tissue Organ Cult 110:69-76. doi: $10.1007 / \mathrm{s} 11240-012-0131-\mathrm{z}$

Zaki M, Dickinson H (1995) Modification of cell development in vitro: The effect of colchicine on anther and isolated microspore culture in Brassica napus. Plant Cell Tissue Organ Cult 40:255-270. doi: 10.1007/BF00048132

Zawada AM, Rogacev KS, Müller S, et al (2014) Massive analysis of cDNA Ends (MACE) and miRNA expression profiling identifies proatherogenic pathways in chronic kidney disease. Epigenetics 9:161-172. doi: 10.4161/epi.26931 
Zeng X, Wen J, Wan Z, et al (2010) Effects of Bleomycin on microspore embryogenesis in Brassica napus and detection of somaclonal variation using AFLP molecular markers. Plant Cell, Tissue Organ Cult 101:23-29. doi: 10.1007/s11240-009-9658$\mathrm{Z}$

Zhang, He, Xu, et al (2006a) Genetic analyses of agronomic and seed quality traits of doubled haploid population in Brassica napus through microspore culture. Euphytica 149:169-177. doi: doi: 10.1007/s10681-005-9064-5

Zhang D, Zhao H, Shi L, Xu F (2014a) Physiological and genetic responses to boron deficiency in Brassica napus : A review. Soil Sci Plant Nutr 60:304-313. doi: 10.1080/00380768.2014.893537

Zhang F, Aoki S, Takahata Y (2003) RAPD markers linked to microspore embryogenic ability in Brassica crops. Euphytica 131:207-213. doi: 10.1023/A:1023955131523

Zhang FL, Takahata Y (2001) Inheritance of microspore embryogenic ability in Brassica crops. TAG Theor Appl Genet 103:254-258. doi: 10.1007/s001220100602

Zhang GQ, Zhang DQ, Tang GX, et al (2006b) Plant development from microsporederived embryos in oilseed rape as affected by chilling, desiccation and cotyledon excision. Biol Plant 50:180-186. doi: 10.1007/s10535-006-0004-6

Zhang Y, Peng L, Wu Y, et al (2014b) Analysis of global gene expression profiles to identify differentially expressed genes critical for embryo development in Brassica rapa. Plant Mol Biol 86:425-442. doi: 10.1007/s11103-014-0238-1

Zhang Y, Wang A, Liu Y, et al (2011) Effects of the antiauxin PCIB on microspore embryogenesis and plant regeneration in Brassica rapa. Sci Hortic (Amsterdam) 130:32-37. doi: 10.1016/j.scienta.2011.06.047

Zhang Y, Wang A, Liu Y, et al (2012) Improved production of doubled haploids in Brassica rapa through microspore culture. Plant Breed 131:164-169. doi: 10.1111/j.1439-0523.2011.01927.x

Zhao J, Becker HC, Zhang D, et al (2005) Oil content in a European X Chinese rapeseed population: QTL with additive and epistatic effects and their genotypeenvironment interactions. Crop Sci 45:51-59.

Zhao J, Simmonds D, Newcomb W (1996a) Induction of embryogenesis with colchicine instead of heat in microspores ofBrassica napus L. cv. Topas. Planta 198:433-439. doi: $10.1007 /$ BF00620060

Zhao J, Simmonds DH, Newcomb W (1996b) High frequency production of doubled haploid plants of Brassica napus cv. Topas derived from colchicine-induced microspore embryogenesis without heat shock. Plant Cell Rep 15:668-671.

Zhou W, Hagberg P, Tang G (2002a) Increasing embryogenesis and doubling efficiency by immediate colchicine treatment of isolated microspores in spring Brassica napus. Euphytica 128:27-34.

Zhou WJ, Tang GX, Hagberg P (2002b) Efficient production of doubled haploid plants by immediate colchicine treatment of isolated microspores in winter Brassica napus. Plant Growth Regul 37:185-192. doi: 10.1023/A:1020561201125 
Zrenner R, Stitt M, Sonnewald U, Boldt R (2006) Pyrimidine and Purine Biosynthesis and Degradation in Plants. Annu Rev Plant Biol 57:805-836. doi:

10.1146/annurev.arplant.57.032905.105421

Zuo J, Niu Q-W, Frugis G, Chua N-H (2002) The WUSCHEL gene promotes vegetative-toembryonic transition in Arabidopsis. Plant J 30:349-359. doi: 10.1046/j.1365313X.2002.01289.x

Zur I, Dubas E, Krzewska M, et al (2014) Hormonal requirements for effective induction of microspore embryogenesis in triticale (x Triticosecale Wittm.) anther cultures. Plant Cell Rep 34:47-62. doi: 10.1007/s00299-014-1686-4

Zur I, Dubas E, Krzewska M, Janowiak F (2015) Current insights into hormonal regulation of microspore embryogenesis. Front Plant Sci 6:1-10. doi: $10.3389 /$ fpls.2015.00424 


\section{Appendix}

\section{A.1}

Supplementary Table 1. Mean values over 5 experiments of the evaluated traits. Microspore density (MIC) is expressed as the volume of microspores per $\mathrm{ml}$ of liquid medium. Number of embryos (MDE) is expressed as the quantity of microspore derived embryos per single culture. Microspore embryogenic potential (POT) is defined as the number of microspore derived embryos produced from a certain number of microspores. The trait survival (SURV) is determined as the percentage of microspore derived embryos that were alive after 6 weeks of being transferred to B5 medium. Root (ROOT) regeneration was calculated as the percentage of microspore derived embryos which developed roots from the hypocotyl. Direct embryo to plant conversion (DEPC) was determined as the proportion of microspore derived embryos that regenerated into plants, presenting both shoot and root. The trait secondary embryogenesis (SECEMB) was scored as the percentage of microspore derived embryos that generated somatic embryos.

\begin{tabular}{cccccccc}
\hline DH LINE & MIC & MDE & POT & SURV & SECEMB & ROOT & DEPC \\
\hline TG-004 & 1102488 & 2867.25 & 0.303 & 43.214 & 15.714 & 36.786 & 26.786 \\
TG-005 & 1008651 & 24520.6 & 2.315 & 76.894 & 65.909 & 79.412 & 66.288 \\
TG-006 & 1184748 & 31244.6 & 3.382 & 98.438 & 47.083 & 86.429 & 72.500 \\
TG-007 & - & 6350.6 & - & 89.815 & 72.685 & 54.167 & 37.963 \\
TG-008 & 1709184 & 40656.2 & 2.545 & 87.109 & 14.844 & 76.071 & 71.324 \\
TG-009 & 1519716 & - & - & 57.143 & 24.554 & - & - \\
TG-011 & - & 5227.75 & - & 88.942 & 43.750 & 74.519 & 67.308 \\
TG-012 & 1298336 & 16586.8 & 1.452 & 97.917 & 32.661 & 83.333 & 75.379 \\
TG-013 & 745941 & 18703.33 & 2.918 & 99.554 & 62.054 & 76.339 & -
\end{tabular}




\begin{tabular}{|c|c|c|c|c|c|c|c|}
\hline TG-016 & & - & - & - & - & - & 56.696 \\
\hline TG-018 & 1631600 & 15995.25 & 0.822 & 86.538 & 43.750 & 75.962 & 58.654 \\
\hline TG-019 & 1056507 & 7950 & 0.892 & 97.794 & 71.691 & 88.235 & 70.221 \\
\hline TG-020 & 897751.2 & 378.2 & 0.044 & - & - & - & - \\
\hline TG-021 & 1195832 & 2557 & 0.214 & 93.750 & 78.646 & 79.688 & 36.458 \\
\hline TG-022 & 1120732 & 1147 & 0.037 & 85.294 & - & 78.241 & 38.603 \\
\hline TG-023 & 745941 & 4693.2 & 0.704 & 85.000 & 20.417 & 77.574 & 79.435 \\
\hline TG-024 & 801876 & 36354.5 & 3.918 & 62.879 & 21.970 & - & 42.803 \\
\hline TG-025 & 1120003 & 1359.2 & 0.189 & 75.463 & 27.652 & 72.727 & 57.500 \\
\hline TG-026 & 729168 & 2708.6 & 0.132 & 47.500 & 6.786 & - & - \\
\hline TG-027 & 1051752 & 14039 & 1.532 & 90.741 & 60.185 & 79.167 & 54.630 \\
\hline TG-028 & 806750.4 & 5813.6 & 0.845 & 46.875 & 10.156 & - & 32.857 \\
\hline TG-029 & 1030500 & 9390 & 0.828 & 77.083 & 4.435 & 79.167 & 47.059 \\
\hline TG-030 & 1124753 & 7426.6 & 0.655 & 90.625 & - & - & 60.938 \\
\hline TG-031 & 724255.2 & 23614 & 2.056 & 79.412 & 37.868 & 66.912 & 58.214 \\
\hline TG-032 & 1064009 & 5215 & 0.298 & 92.803 & 54.412 & 90.278 & 71.591 \\
\hline TG-033 & 1148652 & 13193 & 1.115 & 96.212 & - & 94.231 & 66.667 \\
\hline TG-034 & 911503.2 & 14267 & 1.467 & 69.643 & 13.571 & - & 45.357 \\
\hline TG-035 & 1058632 & 18232 & 1.546 & - & - & - & - \\
\hline TG-036 & 1087188 & 24443.6 & 2.099 & 84.848 & 20.703 & 82.197 & 43.214 \\
\hline TG-037 & 1014375 & 10821.2 & 1.060 & 65.152 & 26.136 & 51.515 & 59.848 \\
\hline TG-038 & - & 40280.6 & - & 77.941 & 11.029 & - & 52.941 \\
\hline TG-039 & 903014.4 & 4958.6 & 0.545 & 63.636 & 39.394 & 52.273 & 35.606 \\
\hline TG-040 & 1201500 & 1780.2 & 0.149 & 60.294 & - & 53.676 & 21.324 \\
\hline TG-041 & 754250.4 & 8945.6 & 1.590 & 99.632 & 84.926 & 86.397 & 63.235 \\
\hline TG-042 & 1227920 & 12928 & 1.102 & 41.071 & 16.786 & 13.603 & 28.214 \\
\hline TG-043 & 998011.2 & 38027.2 & 1.988 & 26.172 & 14.063 & 25.357 & 15.000 \\
\hline TG-044 & 916566 & 463.2 & 0.062 & 95.089 & 29.911 & 87.946 & 85.268 \\
\hline TG-045 & 1339875 & 20903 & 1.450 & 68.382 & 12.868 & 71.786 & 48.529 \\
\hline TG-050 & - & 0.0 & $0.000^{*}$ & - & - & - & - \\
\hline TG-051 & 1279246 & 22878 & 1.932 & 88.710 & 84.677 & 65.323 & 22.581 \\
\hline TG-053 & 1265254 & 5843.6 & 0.422 & 55.645 & 13.603 & 27.941 & 26.103 \\
\hline TG-054 & 1314250 & 22018.75 & 1.491 & 96.371 & 39.113 & 81.048 & 74.194 \\
\hline TG-059 & 1279683 & 13069.8 & 1.170 & 81.250 & 60.156 & 52.734 & 32.422 \\
\hline TG-061 & 1220622 & 18347.2 & 2.011 & 78.409 & 63.636 & 51.894 & 34.091 \\
\hline TG-063 & 922916 & 36174.4 & 1.561 & 86.765 & 44.907 & 80.556 & 73.214 \\
\hline TG-064 & 1331750 & 329.6 & 0.027 & 61.290 & - & 65.530 & 38.636 \\
\hline TG-065 & 1398250 & 110 & 0.007 & 89.643 & 82.738 & - & 53.929 \\
\hline TG-066 & 1045626 & 6492.4 & 0.559 & 56.250 & 21.691 & 73.276 & 28.309 \\
\hline TG-069 & 1737747 & 7507.8 & 0.409 & 94.737 & 82.237 & 90.789 & 75.658 \\
\hline TG-071 & 1253439 & 6558.75 & 0.536 & 57.212 & 12.500 & - & - \\
\hline TG-073 & 1087248 & 15353.5 & 2.447 & 40.234 & 19.922 & 28.906 & 24.219 \\
\hline TG-074 & 1013292 & 5698.8 & 0.532 & 90.152 & 40.530 & 71.970 & 58.333 \\
\hline TG-075 & - & 10975.6 & - & 81.429 & 28.214 & 71.429 & 64.286 \\
\hline TG-076 & 904584 & 1206.4 & 0.116 & 33.173 & 8.173 & 30.921 & 22.596 \\
\hline TG-078 & 1174256 & 2598.25 & 0.244 & 64.286 & 13.839 & 45.833 & 49.107 \\
\hline TG-079 & 775420 & 4630.333 & 0.606 & 74.242 & 26.136 & 54.167 & 44.318 \\
\hline
\end{tabular}




\begin{tabular}{|c|c|c|c|c|c|c|c|}
\hline TG-083 & 1085336 & 19957.33 & 1.776 & - & - & - & - \\
\hline TG-089 & 1319052 & 3387.75 & 0.225 & 80.000 & 46.500 & 66.000 & 53.500 \\
\hline TG-090 & 1099001 & 2103.6 & 0.237 & 76.838 & 34.559 & 76.429 & 70.714 \\
\hline TG-091 & 1205671 & 13819.2 & 1.133 & 76.563 & 19.141 & 52.344 & 48.438 \\
\hline TG-093 & 1280998 & 5127.4 & 0.493 & 48.162 & 20.370 & 35.662 & 23.162 \\
\hline TG-095 & 1292814 & 35243.6 & 2.861 & 64.286 & 29.643 & 58.929 & - \\
\hline TG-099 & 1157784 & - & - & - & - & - & - \\
\hline TG-101 & 920916 & 22632.6 & 1.292 & 92.647 & 56.985 & 88.971 & 60.357 \\
\hline TG-102 & - & 0.0 & $0.000^{*}$ & - & - & - & - \\
\hline TG-103 & - & 0.0 & $0.000^{*}$ & - & - & - & - \\
\hline TG-104 & 909998.4 & 17801.8 & 2.064 & 88.750 & 55.417 & 64.453 & 49.583 \\
\hline TG-106 & 1087868 & 2214 & 0.170 & - & - & - & - \\
\hline TG-107 & 1433160 & 38433 & 2.952 & 66.912 & 35.662 & - & 34.559 \\
\hline TG-112 & - & 292.8 & $0.002^{* *}$ & 81.522 & 61.413 & 63.043 & 46.739 \\
\hline TG-113 & 1212790 & 5899.25 & 0.636 & 95.703 & 51.563 & 93.214 & 78.929 \\
\hline TG-114 & 1351162 & 284 & 0.021 & 91.250 & - & 67.500 & - \\
\hline TG-116 & 1130940 & 5230.2 & 0.577 & 97.500 & - & 77.500 & - \\
\hline TG-118 & 1093728 & 18920.8 & 1.246 & 90.714 & 72.685 & 81.481 & 49.286 \\
\hline TG-119 & 1001874 & 32900.2 & - & 52.652 & 16.827 & - & 39.015 \\
\hline TG-120 & 1203126 & 26714 & - & 93.145 & 54.032 & 92.188 & 69.355 \\
\hline TG-121 & 1234001 & 1319.6 & 0.088 & 85.357 & 48.214 & 83.333 & 23.214 \\
\hline TG-122 & 1496284 & 8599.667 & 0.549 & - & - & - & - \\
\hline TG-125 & 1380309 & 633.75 & 0.047 & - & - & - & - \\
\hline TG-128 & 1265000 & 13207 & - & 82.857 & 18.571 & 95.357 & 78.214 \\
\hline TG-129 & 943248 & 84.25 & 0.313 & 92.411 & 23.214 & 70.982 & - \\
\hline TG-132 & 1456253 & 1884.8 & 0.174 & 80.804 & 63.393 & 76.786 & 44.643 \\
\hline TG-133 & 1204584 & 23046 & - & 92.411 & 32.589 & 69.643 & 49.107 \\
\hline TG-134 & 918753.6 & 8518.2 & 1.093 & 71.691 & 12.891 & 27.273 & 73.162 \\
\hline TG-135 & 1148751 & 5838.75 & - & 87.946 & 34.375 & 84.524 & 63.839 \\
\hline TG-136 & 957052.8 & 14766.4 & 1.209 & 84.191 & 75.962 & 73.558 & - \\
\hline TG-137 & 1297502 & 9792.6 & 0.834 & 80.147 & 11.250 & - & 31.985 \\
\hline TG-138 & - & 8986.4 & - & 98.611 & 10.648 & 84.722 & 81.944 \\
\hline TG-139 & 1170710 & 11027.75 & 0.947 & 84.500 & 76.000 & 74.500 & - \\
\hline TG-145 & 910113 & 4943.5 & 0.396 & - & - & - & - \\
\hline TG-149 & 1174250 & 5861.2 & 0.504 & 75.000 & 42.500 & 57.721 & 56.618 \\
\hline TG-151 & 1312502 & 21090 & 1.684 & 87.121 & 29.924 & 70.076 & 62.121 \\
\hline TG-158 & - & 1479.5 & - & 54.167 & 43.056 & 44.907 & 24.537 \\
\hline TG-162 & 1055836 & 500 & 0.104 & - & - & - & - \\
\hline TG-163 & 1408664 & 7903 & 0.474 & 68.015 & 19.853 & 43.182 & 35.227 \\
\hline TG-164 & 1391249 & 293.8 & 0.027 & 60.526 & - & - & - \\
\hline TG-165 & 1098999 & 7642.2 & 0.614 & 76.389 & 50.926 & 61.111 & 45.370 \\
\hline TG-166 & - & 168.8 & $0.019^{* *}$ & - & - & - & - \\
\hline TG-167 & 1071876 & 18460 & 1.752 & 87.500 & 31.250 & 73.162 & 72.059 \\
\hline TG-168 & - & 0.000 & $0.000^{*}$ & - & - & - & - \\
\hline TG-169 & 1385432 & 20207.6 & 1.388 & 90.625 & 44.643 & 78.125 & 68.304 \\
\hline TG-170 & 1344732 & 889.6 & 0.111 & 77.941 & 55.147 & 32.353 & - \\
\hline TG-171 & 917001.6 & 1651.8 & 0.103 & 35.227 & 15.909 & 22.159 & 13.068 \\
\hline
\end{tabular}




$\begin{array}{lcclllll}\text { TG-172 } & 1485318 & 15330.75 & 0.547 & 61.574 & - & 50.463 & 40.741 \\ \text { TG-182 } & 1248101 & 31483 & 3.174 & 47.596 & 18.269 & 38.942 & 32.212 \\ \text { TG-188 } & 1462916 & 33850 & 0.981 & 91.667 & 18.056 & 81.019 & 73.148 \\ \text { TG-193 } & 1382504 & - & - & - & - & - & - \\ \text { TG-196 } & 1784998 & 8902.6 & 0.475 & 98.611 & 91.250 & 67.593 & 46.759 \\ \text { TG-198 } & 1289500 & 9750.6 & 0.467 & 93.214 & 36.071 & 66.429 & 57.143 \\ \text { TG-199 } & 1435001 & 3350.6 & 0.261 & 64.815 & 49.537 & 46.296 & 22.685 \\ \text { TG-201 } & 1286249 & 1782 & 0.104 & 70.625 & - & - & 34.375 \\ \text { TG-204 } & - & 4499.5 & - & 57.143 & 24.554 & 40.179 & 36.607\end{array}$

* The microspore embryogenic potential was calculated as 0.000 when, even if the data for microspore density was not available, the number of regenerated embryos was null.

** The mean of the microspore embryogenic potential was calculated based of 5 experiments from which 4 did not developed embryos, therefore the microspore embryogenic potential was calculated even though the microspore density was not available. 


\section{Curriculum vitae}

PERSONAL

DETAILS

Name

Ariana Istar Valdés Velázquez

Place of birth

Mexico City, Mexico

Date of birth

05. May 1982

Address

Cerrada Cipreses 8, Colonia Ampliación Oriente, 14400 Tlalpan, Distrito Federal, Ciudad de Mexico, Mexico

Email

Nationality

a.valdesvelazquez@stud.uni-goettingen.de

Mexican

Marital status

Single

\section{EDUCATION}

Oct $2012-$ Nov

2016

Oct $2008-$ Nov

2010

Jan 2004 - Oct

2006

International PhD Program for Agricultural Science Faculty of Agricultural Sciences, Division of Plant Breeding, Georg-AugustUniversität Göttingen, Germany

MSc in Biological Sciences (Experimental Biology), Institute of Biology, Universidad Nacional Autónoma de México (UNAM), Mexico

BSc in Biology, Faculty of Sciences, Universidad Nacional Autónoma de México (UNAM), Mexico

\section{WORK}

\section{EXPERIENCE}

May - Aug 2012

Development of the project BIOPUNTIA: Opuntia ssp. as a source of pharmaceutical compounds to develop new products. Faculty of Chemical Sciences, Universidad Autónoma de San Luis Potosí, Mexico

Sep 2009 - Aug 2012

Senior Lecturer at UNAM. Bachelor Degree in Biology, Faculty of Sciences, Universidad Nacional Autónoma de México (UNAM), Mexico 
Workshop on Molekulare Züchtung und Somatische Embryogenese (Molecular Breeding and Somatic Embryogenesis). KWS SAAT SE, Einbeck, Germany

\section{PUBLICATIONS DURING PhD STUDIES}

2016 Characterization of genomic regions responsible for microspore embryogenesis and direct embryo to plant conversion in the $\mathrm{DH}$ oilseed rape population DH4079 x Express 617. German Society for Plant Breeding (GPZ) Conference, 08 - 1 March 2016, Bonn, Germany

Valdés-Velázquez AI, Clemens R, Möllers C (2015) Characterization of genomic regions responsible for microspore embryogenic potential and direct embryo to plant conversion of a doubled haploid oilseed rape population. Proc 14th International Rapeseed Congress, 05 - 09 July 2015, Saskatoon, Saskatchewan, Canada

Valdés-Velázquez AI, Mahasuk P, Clemens R, Möllers C (2014) Inheritance of microspore embryogenesis and direct embryo to plant conversion in a DH population derived from the cross DH4079 x Express 617 (Brassica napus L.). 17th Conference of the Genome Research Working Group of the German Society for Plant Breeding (GPZ), 11 - 13 February 2014, Cologne, Germany

Valdés-Velázquez AI, Mahasuk P, Clemens R, Möllers C (2014) Characterization of genomic regions responsible for microspore embryogenesis and direct embryo to plant conversion in the doubled haploid oilseed rape population DH4079 x Express 617 (Brassica napus L.). The 2012 German Society for Plant Breeding (GPZ) Conference "Genetic Variation in Plant Breeding", 23 - 25 September 2014, Kiel, Germany 